\title{
The lithium content of the Galactic Halo stars ${ }^{\star}$
}

\author{
C. Charbonnel ${ }^{1,2}$ and F. Primas ${ }^{3,2, \star \star}$
}

\author{
1 Geneva Observatory, 51 chemin des Maillettes, 1290 Sauverny, Switzerland \\ e-mail: Corinne.Charbonnel@obs . unige.ch \\ 2 Laboratoire d'Astrophysique de Toulouse et de Tarbes, CNRS UMR 5572, 14 Av. E. Belin, 31400 Toulouse, France \\ 3 European Southern Observatory, Karl-Schwarzschild Str. 2, 85748 Garching b. München, Germany \\ e-mail: fprimas@eso.org
}

Received 7 December 2004 / Accepted 8 May 2005

\section{ABSTRACT}

Thanks to the accurate determination of the baryon density of the universe by the recent cosmic microwave background experiments, updated predictions of the standard model of Big Bang nucleosynthesis now yield the initial abundance of the primordial light elements with unprecedented precision. In the case of ${ }^{7} \mathrm{Li}$, the $\mathrm{CMB}+\mathrm{SBBN}$ value is significantly higher than the generally reported abundances for Pop II stars along the so-called Spite plateau. In view of the crucial importance of this disagreement, which has cosmological, galactic and stellar implications, we decided to tackle the most critical issues of the problem by revisiting a large sample of literature Li data in halo stars that we assembled following some strict selection criteria on the quality of the original analyses.

In the first part of the paper we focus on the systematic uncertainties affecting the determination of the Li abundances, one of our main goal being to look for the "highest observational accuracy achievable" for one of the largest sets of Li abundances ever assembled. We explore in great detail the temperature scale issue with a special emphasis on reddening. We derive four sets of effective temperatures by applying the same colour $-T_{\text {eff }}$ calibration but making four different assumptions about reddening and determine the LTE lithium values for each of them. We compute the NLTE corrections and apply them to the LTE lithium abundances. We then focus on our "best" (i.e. most consistent) set of temperatures in order to discuss the inferred mean $\mathrm{Li}$ value and dispersion in several $T_{\text {eff }}$ and metallicity intervals. The resulting mean $\mathrm{Li}$ values along the plateau for $[\mathrm{Fe} / \mathrm{H}] \leq-1.5$ are $A(\mathrm{Li})_{\mathrm{NLTE}}=2.214 \pm 0.093$ and $2.224 \pm 0.075$ when the lowest effective temperature considered is taken equal to $5700 \mathrm{~K}$ and $6000 \mathrm{~K}$ respectively. This is a factor of $\sim 2.48$ to 2.81 (depending on the adopted SBBN model and on the effective temperature range chosen to delimit the plateau) lower than the CMB+SBBN determination. We find no evidence of intrinsic dispersion. Assuming the correctness of the $\mathrm{CMB}+\mathrm{SBBN}$ prediction, we are then left with the conclusion that the $\mathrm{Li}$ abundance along the plateau is not the pristine one, but that halo stars have undergone surface depletion during their evolution.

In the second part of the paper we further dissect our sample in search of new constraints on Li depletion in halo stars. By means of the Hipparcos parallaxes, we derive the evolutionary status of each of our sample stars, and re-discuss our derived Li abundances. A very surprising result emerges for the first time from this examination. Namely, the mean Li value as well as the dispersion appear to be lower (although fully compatible within the errors) for the dwarfs than for the turnoff and subgiant stars. For our most homogeneous dwarfs-only sample with $[\mathrm{Fe} / \mathrm{H}] \leq-1.5$, the mean $\mathrm{Li}$ abundances are $A(\mathrm{~L})_{\mathrm{NLTE}}=2.177 \pm 0.071$ and $2.215 \pm 0.074$ when the lowest effective temperature considered is taken equal to $5700 \mathrm{~K}$ and $6000 \mathrm{~K}$ respectively. This is a factor of 2.52 to 3.06 (depending on the selected range in $T_{\text {eff }}$ for the plateau and on the SBBN predictions we compare to) lower than the $\mathrm{CMB}+\mathrm{SBBN}$ primordial value. Instead, for the post-main sequence stars the corresponding values are $2.260 \pm 0.1$ and $2.235 \pm 0.077$, which correspond to a depletion factor of 2.28 to 2.52 .

These results, together with the finding that all the stars with Li abnormalities (strong deficiency or high content) lie on or originate from the hot side of the plateau, lead us to suggest that the most massive of the halo stars have had a slightly different Li history than their less massive contemporaries. In turn, this puts strong new constraints on the possible depletion mechanisms and reinforces Li as a stellar tomographer.

Key words. stars: abundances - stars: Population II - stars: evolution - Galaxy: abundances - Galaxy: halo cosmology: early Universe

\section{The lithium paradigm}

From the time of its discovery in the stellar atmospheres of very metal-poor Population II stars (Spite \& Spite 1982a), lithium has been considered a key diagnostic to test and constrain our

^ The whole Tables 3-6, 8, 9 are only available in electronic form at http://www . edpsciences .org

$\star \star$ Visiting Astronomer at the LATT - OMP, Toulouse, France. understanding and description of the primordial Universe, of stellar interiors and evolution, and of spallation physics (for the latter two especially so if combined with abundances of beryllium and boron).

Lithium-7 is one of the four primordial isotopes that have been formed in observable quantities by nuclear reactions during the first minutes of the Universe (e.g. Olive et al. 2000, and references therein). Together with deuterium, helium-3 and 
Table 1. WMAP+SBBN primordial abundances predicted when $\eta=(6.14 \pm 0.25) \times 10^{-10}\left(\right.$ or $\Omega_{\mathrm{b}} h^{2}=0.0224 \pm 0.0009$; Spergel et al. 2003) is adopted.

\begin{tabular}{cccc}
\hline \hline & Coc et al. (2004) & Cyburt (2004) & Serpico et al. (2004) \\
\hline $\mathrm{D} / \mathrm{H}$ & $\left(2.60_{-0.17}^{+0.19}\right) \times 10^{-5}$ & $\left(2.55_{-0.20}^{+0.21}\right) \times 10^{-5}$ & $\left(2.58_{-0.16}^{+0.19}\right) \times 10^{-5}$ \\
$\mathrm{Y}_{\mathrm{P}}$ & $0.2479 \pm 0.0004$ & $0.2485 \pm 0.0005$ & $0.2479 \pm 0.0004$ \\
${ }^{3} \mathrm{He} / \mathrm{H}$ & $(1.04 \pm 0.04) \times 10^{-5}$ & $(1.01 \pm 0.07) \times 10^{-5}$ & $1.03 \pm 0.03$ \\
${ }^{7} \mathrm{Li} / \mathrm{H}$ & $\left(4.15_{-0.45}^{+0.49}\right) \times 10^{-10}$ & $\left(4.26_{-0.86}^{+0.91} \times\right) 10^{-10}$ & $\left(4.6_{-0.4}^{+0.4}\right) \times 10^{-10}$ \\
\hline
\end{tabular}

helium-4, knowledge of its primordial abundance provides one of the main observational constraints on the baryon-to-photon ratio $\eta \propto \Omega_{\mathrm{b}} h^{2}$ which is the only free parameter of the standard Big Bang Nucleosynthesis (SBBN).

Among these light elements, lithium is one of the easiest to observe (its resonant doublet falls at $670 \mathrm{~nm}$, i.e. easily accessible from ground telescopes even of small sizes), which explains the wealth of data available in the literature.

Another reason for such a large literature database is connected to the important finding of a remarkably flat and constant Li abundance among Galactic halo dwarf stars spanning a wide range of effective temperatures and metallicities (the so-called Spite plateau, cf. Spite \& Spite 1982a,b). This result came as a surprise. At that time indeed it was generally believed that the primordial $A(\mathrm{Li})^{1}$ abundance was in the range 3.0-3.3, which corresponds to the value measured in meteorites and also to the maximum value detected in Population I stars, both in the field and in open clusters (see the review by Boesgaard \& Steigman 1985). If this were the case, then the "constant" but lower value of lithium along the Spite plateau would have required that all the oldest stars of our Galaxy had suffered a uniform depletion by about a factor of 10. Although several mechanisms could be conjectured to modify the surface lithium abundance (proto-stellar destruction, microscopic diffusion, turbulent diffusion, mass loss), they were also suspected to depend on the stellar mass (i.e., on the effective temperature). Consequently the constancy of the Li plateau was used (and is actually still used very often) as an argument to say that these processes were in fact not efficient in Pop II stars.

The other interpretation was then that the plateau value represents the amount of $\mathrm{Li}$ produced during the Big Bang, and that the Galaxy had been enriched in its Li content by a factor of at least 10 since its birth ${ }^{2}$. The fact that lithium is produced in several other nucleosynthetic sites (i.e. $\alpha-\alpha$ fusion, spallation reactions, late stellar evolutionary stages, like AGB stars, novae, etc.; see Romano et al. 2003, and references therein), none of which has been quantitatively and accurately estimated nor strongly constrained by observations, complicates the final interpretation of its Galactic evolution.

Recent results on cosmic microwave background anisotropies, most particularly from the Wilkinson Microwave Anisotropy Probe (WMAP) experiment (Bennet et al. 2003; Spergel et al. 2003) allowed an unprecedented precision on the

\footnotetext{
${ }^{1} A(\mathrm{Li})=12+\log N(\mathrm{Li}) / N(\mathrm{H})$.

2 Actually the primordial Li abundance could even be lower than the plateau value because of production in the early Galaxy (Ryan et al. 1999; Suzuki et al. 2000).
}

determination of the baryon-to-photon ratio $\eta$ and revealed that Li seems to lie between the two extreme solutions discussed above. The WMAP data alone lead to $\Omega_{\mathrm{b}} h^{2}=0.0237 \pm 0.001$, or $\eta=6.5_{-0.3}^{+0.4} \times 10^{-10}$. When combined with additional CMB experiments (CBI, Pearson et al. 2003; ACBAR, Kuo et al. 2002) and with measurements of the power spectrum ( $2 \mathrm{dF}$ Galaxy Redshift Survey, Percival et al. 2001; Ly $\alpha$ forest, Croft et al. 2002; Gnedin \& Hamilton 2002), the resulting values are $\Omega_{\mathrm{b}} h^{2}=0.0224 \pm 0.0009$ or $\eta=6.1_{-0.2}^{+0.3} \times 10^{-10}$. With this value of $\eta$, updated SBBN predictions now allow a precise determination of the primordial abundances of the light elements $\mathrm{D},{ }^{3} \mathrm{He},{ }^{4} \mathrm{He}$ and ${ }^{7} \mathrm{Li}$ that we can compare with observations in low-metallicity environments. The $W M A P+\mathrm{SBBN}$ determinations of these abundances in the most two recent studies (Coc et al. 2004; Cyburt 2004; Serpico et al. 2004) are summarised in Table 1.

A very good agreement is achieved between the primordial abundance of deuterium derived from $W M A P+\mathrm{SBBN}$ and the average value of $\mathrm{D} / \mathrm{H}$ observations in cosmological clouds along the line of sight of quasars (Kirkman et al. 2003). On the other hand the observational data of ${ }^{3} \mathrm{He}$ in galactic HII regions are scarce and must be corrected for contamination of the observed gas by ejecta from earlier generations of stars (e.g. Tosi 1998; Charbonnel 2002). The upper limit to the primordial abundance recommended by Bania et al. (2002) is however quite consistent with the CMB-derived value. Finally the CMB-predicted primordial ${ }^{4} \mathrm{He}$ abundance is higher than the values derived from the determinations in complex lowmetallicity HII regions (both galactic and extra-galactic) and the extrapolation to zero oxygen abundance (Izotov \& Thuan 2004; Olive \& Skillman 2004 and references therein). However the difference is relatively modest $(2-3 \%)$ and it may simply call for further exploration of the systematic effects in the abundance analysis.

The most critical case concerns ${ }^{7} \mathrm{Li}$, the CMB-derived primordial abundance of which is clearly higher (by about a factor of 3 ) than the current determinations in low-metallicity halo stars. This result seems to be very robust with respect to the nuclear uncertainties on the SBBN reactions although Coc et al. (2004) show that the discrepancy could be resolved by an increase of a factor of $\sim 100$ of the ${ }^{7} \mathrm{Be}(\mathrm{d}, \mathrm{p}) 2^{4} \mathrm{He}$ reaction rate. Although this is not supported by the data currently available, this issue has to be further investigated experimentally. Should this nuclear solution be excluded, we would then be left with the astrophysical solution. Namely, with the conclusion that the $\mathrm{Li}$ abundance that we see at the surface of halo stars is not the pristine one, but that these stars have undergone surface lithium 
depletion at some point during their evolution. This possibility has been discussed many times in the literature. Several physical mechanisms have been invoked, but all the current models encounter considerable difficulties to reconcile a non negligible depletion of lithium with both the flatness and the small dispersion along the so-called $\mathrm{Li}$ plateau (see the review by Pinsonneault et al. 2000; and Talon \& Charbonnel 2004, for more recent references). The challenge thus still remains to identify the process (or processes) by which a reduction by a factor of $\sim 3$ could occur so uniformly in stars over a large range in effective temperature and metallicity.

With the CMB constraint, we are now entering a golden age for $\mathrm{Li}$ as both a baryometer and a stellar tomographer ${ }^{3}$. In this quest however one has still to pay special attention to the observational analysis and determination of the lithium abundances in the most metal-poor, thus the oldest stars of our Galaxy. As a matter of fact and despite the large number of spectroscopic data that has become available in the last two decades, there are still on-going debates on the patterns of the plateau, like its thinness, the possible existence of a spread and of a dependence of $A(\mathrm{Li})$ with metallicity and effective temperature. These characteristics must be precisely determined in order to constrain the physical processes which lead to Li depletion in Pop II stars as well as those of Galactic production.

\section{The "Lithium plateau debate"?}

Deliyannis et al. (1993) were among the first to present evidence for the existence of dispersion (of the order of $\pm 20 \%$ about the mean, derived from the "equivalent width-colour" plane), followed by Thorburn (1994) and Norris et al. (1994), the latter being the first to also have found a dependence of $A(\mathrm{Li})$ on both $T_{\text {eff }}$ and metallicity. Molaro et al. (1995) counter-argued these findings showing that when a fully consistently determined temperature scale is used (in their case, the Fuhrmann et al. 1994 scale, derived from Balmer lines fitting), no dispersion nor tilt is found ( $\mathrm{Li}$ abundances are mostly sensitive to $T_{\text {eff }}$ ), but they were shortly followed by Ryan et al. (1996) who once again confirmed the slopes. They argued that the Molaro et al. sample was plagued by the inclusion of subgiants that may have affected their final outcome. On the intrinsic scatter issue, they noted that there were some stars, characterized by very similar parameters (colour, $T_{\text {eff }}$ and metallicity), but that turned out from multiple measurements to have very different $\mathrm{Li}$ abundances. The debate kept being very alive: Spite et al. (1996) explored further the $T_{\text {eff }}$ scale issue (comparing different $T_{\text {eff }}$ determinations) and found that the rms scatter of the $\mathrm{Li}$ abundance was between 0.06 and $0.08 \mathrm{dex}$, hence very small if real. Bonifacio \& Molaro (1997) re-selected their

\footnotetext{
${ }^{3}$ Since lithium is destroyed at quite low temperatures (for stellar interiors) of the order of $2.5 \times 10^{6} \mathrm{~K}$, it is a powerful tool to identify the mechanisms active in stellar interiors and responsible for convective and/or radiative transports, mixing, diffusion, presence of gravitational waves. Together with beryllium and boron, that burn at $3.5 \times 10^{6} \mathrm{~K}$ and $5.0 \times 10^{6} \mathrm{~K}$ respectively, lithium abundances allow us to make a stellar tomography of the external atmospheric layers where these three light nuclides are "nuclearly" preserved (since the epoch of formation when looking at unevolved objects).
}

sample, this time excluding possible outliers (like the abovementioned subgiants) and once again came to the conclusion of no intrinsic dispersion nor dependence of $A(\mathrm{Li})$ on metallicity, but of a tiny trend with the temperature. They concluded that the finding or not of a trend with effective temperature may well depend on the adopted $T_{\text {eff }}$ scale.

It is only towards the end of the 90s when a full agreement on the absence of intrinsic dispersion was reached: Ryan et al. (1999), by analysing a new sample of 23 stars covering a narrow range in $T_{\text {eff }}(6050-6350 \mathrm{~K})$ and in metallicity $(-3.5-$ -2.5 ), claimed that the intrinsic spread is effectively zero, i.e. $0.031 \mathrm{dex}$, at the $1 \sigma$ level (to be compared to their formal errors of $0.033 \mathrm{dex})$. However, they still recovered the dependence on metallicity, at the level of $\mathrm{d} A(\mathrm{Li}) / \mathrm{d}[\mathrm{Fe} / \mathrm{H}]=0.118 \pm 0.023(1 \sigma)$ dex per dex, i.e. very similar to the slopes previously found. The trend with $T_{\text {eff }}$, if any, is likely to be meaningless because of the very narrow $T_{\text {eff }}$ range there explored.

This is when we started developing our project. By comparing the data samples analysed by different authors (starting with the Bonifacio \& Molaro 1997; and Ryan et al. 1996, 1999, because of their final, opposite claims) we noticed that for some stars, common to several analyses, very discrepant $\mathrm{Li}$ abundances were reported, which could have clearly influenced some of the early claims for dispersion, and they could still play a role in the current debate about the existence of a slope between $\mathrm{Li}$ and $T_{\text {eff }}$ and $[\mathrm{Fe} / \mathrm{H}]$.

In order to further tackle these issues, we decided to reanalyse the large sample of $\mathrm{Li}$ abundances available in the literature (Sects. 3 and 4) from a different perspective. One of our main goals is to focus on the systematic uncertainties affecting the determination of Li abundances. First, because the Li abundance is strongly dependent on the assumed temperature, we explore further the temperature scale issue (Sect. 5) with the aim of deriving what the best achievable accuracy may be for a temperature scale derived in a consistent manner. We put special emphasis on reddening, usually an underestimated source of error. We derive four sets of effective temperatures by applying the same colour $-T_{\text {eff }}$ calibration but making four different assumptions about reddening. We then derive the LTE lithium abundances for each of these sets and compute the NLTE corrections. Then we select our "best" (in terms of consistency) set of temperatures in order to determine the mean $\mathrm{Li}$ abundance and the dispersion for one of the largest sample of halo stars ever studied in a consistent way (Sects. 6 and 7). This allows us to derive preliminary results on the mean lithium abundance and dispersion which can be compared to previous analyses (Sect. 8).

Secondly we look afresh at our $\mathrm{Li}$ abundances together with the evolutionary status of each target (Sect. 9) in order to get clues on the internal processes that may have been involved in modifying the Li abundances along the Plateau. This is why we did not restrict a priori our sample to any specific evolutionary status. We then discuss the lithium abundance along the plateau for the dwarf stars only (Sect. 10) and look at its behaviour in subgiants (Sect. 11). We test whether our results on the dispersion and trends can be altered by the presence of binary stars in the sample (Sect. 12), and we finally inspect the cases of stars with extreme lithium abundances (Sect. 13). Then we discuss 
Table 2. Literature sources.

\begin{tabular}{lcccc}
\hline \hline Authors & $R$ & $S / N$ & $T / \mathrm{g} / \mathrm{Fe}^{a}$ & Note $^{b}$ \\
\hline 1. Ryan et al. (1996) & 38000 & $>100$ & $\mathrm{p} / \mathrm{l} / \mathrm{l}$ & $\mathrm{O}, \mathrm{T}$ \\
2. Ryan et al. (1999) & 40000 & $>100$ & $\mathrm{p} / \mathrm{l} / \mathrm{l}$ & $\mathrm{T}$ \\
3. Ryan et al. (2001a,b) & 50000 & $>100$ & $\mathrm{p} / \mathrm{l} / \mathrm{l}$ & $\mathrm{O}, \mathrm{T}$ \\
4. Bonifacio \& Molaro (1997) & $\ldots$ & $\ldots$ & $\mathrm{p} / \mathrm{l} / \mathrm{l}$ & $\mathrm{T}$ \\
5. Pilachowski et al. (1993) & 30000 & $>100$ & $\mathrm{p} / \mathrm{p} / \mathrm{s}$ & $\mathrm{O}$ \\
6. Hobbs \& Thorburn (1991) & 30000 & $>100$ & $\mathrm{l} / \mathrm{l} / \mathrm{l}$ & $\mathrm{O}, \mathrm{T}$ \\
7. Thorburn (1994) & 28000 & $>80$ & $\mathrm{p} / \mathrm{l} / \mathrm{l}$ & $\mathrm{O}$ \\
8. Molaro et al. (1995) & $\ldots$ & $\ldots$ & $\mathrm{p} / \mathrm{l} / \mathrm{l}$ & $\mathrm{T}$ \\
9. Spite et al. (1996) & $\ldots$ & $>80$ & $\mathrm{p} / \mathrm{p} / \mathrm{s}$ & $\mathrm{T}$ \\
10. Ryan \& Deliyannis (1998) & 42000 & $>70$ & $\mathrm{p} / \mathrm{p} / \mathrm{s}$ & $\mathrm{O}$ \\
11. Gutierrez et al. (1999) & 30000 & $>100$ & $\ldots$ & $\mathrm{O}$ \\
12. Fulbright (2000) & 50000 & $>100$ & $\mathrm{~s} / \mathrm{s} / \mathrm{s}$ & $\mathrm{O}$ \\
13. Ford et al. (2002 ) & 50000 & $>150$ & $\mathrm{l} / \mathrm{l} / 1$ & $\mathrm{O}$ \\
\hline
\end{tabular}

${ }^{a} T_{\text {eff }} / \log g /[\mathrm{Fe} / \mathrm{H}]: 1$ = literature; $\mathrm{s}=$ spectroscopy; $\mathrm{p}=$ photometry.

${ }^{b} \mathrm{O}=$ new set of observations; $T=$ new $T_{\text {eff }}$ scale (with Li $E W$ collected from literature).

the current status of Pop II stellar models in view of our observational results (Sect. 14). Finally we summarise our results and conclude on some remaining open questions (Sect. 15).

\section{Sample selection: a critical analysis of the literature}

The database of Li abundances measured in Galactic stars and available in the literature is huge. During this work, we restricted our search to the main observational analyses from the early 90 onwards.

We assembled our final data sample from 13 literature sources (cf. Table 2). The main ones were the works by Ryan et al. (1996, 1999, hereafter R96, R99), Bonifacio \& Molaro (1997, hereafter BM97), and Pilachowski et al. (1993, hereafter PSB93), the last one in order to include a large set of subgiant stars. R96, R99, and PSB93 analysed newly observed spectra and derived new temperatures, whereas BM97 collected a large sample of stars for which the equivalent width of the Li I line had already been measured and re-computed the Li abundance based on a new temperature scale. This first list of targets was then complemented by objects taken from Hobbs \& Thorburn (1991), Thorburn (1994, for those very few stars which had not been already re-analysed by Ryan et al. 1996), Molaro et al. (1995), Spite et al. (1996), Ryan \& Deliyannis (1998), Gutierrez et al. (1999), Fulbright (2000), Ryan et al. (2001a,b), and Ford et al. (2002). We gave our preference to original works, i.e. works that added new observations or that re-analysed literature values based on a new temperature scale. This exercise left us with a sample of 146 stars, covering the metallicity range between $[\mathrm{Fe} / \mathrm{H}]=-1.0$ and -3.5 , the temperature interval $T_{\text {eff }}=4500-6500 \mathrm{~K}$, and the surface gravity range between $\log g=3.0-5.0$. In other words, we are sampling the main sequence, subgiant and giant evolutionary stages. Table $3^{4}$ presents the data sample and its main characteristics in

\footnotetext{
${ }^{4}$ Available in its entirety on-line.
}

terms of nomenclature (including cross-identifications), stellar parameters and the equivalent widths of the Li I line as found in the literature. For those objects, for which multiple determinations are available, the minimum and maximum values are listed. For a more detailed comparison, Fig. 1 shows how the equivalent widths measured (used) by some of the literature sources listed in Table 1 compare to each other. For the purpose of this test, we selected those works that had the largest number of stars in common.

For completion, we note that there have been three other recent works that have also made use of the large database of Li measurements available from the literature. Two of them had different scientific goals and they both used (after a critical selection) the $\mathrm{Li}$ abundances as found in the literature: Romano et al. (1999) re-assessed the Galactic evolution of lithium, whereas Pinsonneault et al. (2002) compared the most recent $\mathrm{Li}$ abundances to theoretical predictions of models including rotational mixing and examined them for trends with metallicity. This is why they do not appear in our list of literature sources. The third, most recent and most similar work to ours is the one from Meléndez \& Ramírez (2004), who studied the behavior of the $A(\mathrm{Li})$ plateau and its trends in a sample of 41 dwarf stars. An improved InfraRed Flux Method-based temperature scale was derived (Ramírez \& Meléndez 2005a,b, hereafter RM05a,b) and used to compute the Li abundances (from equivalent widths taken from the literature). Because RM05a,b became public at a late stage in our refereeing process, we did not update our input targets list, but instead we decided to discuss and compare their and our results when relevant.

\section{Stellar parameters: I. Gravity, metallicity, and microturbulence}

Analysing lithium is not very difficult. Lithium appears in a stellar absorption spectrum with few transitions, namely the resonant line at $670.7 \mathrm{~nm}$, and a much weaker signature at $610.4 \mathrm{~nm}$, only recently explored in the most metal-poor stars (cf. Bonifacio \& Molaro 1998; Ford et al. 2002). The $670.7 \mathrm{~nm}$ line falls in a clean spectral region, especially in metal-deficient stars. From its equivalent width it is easy to derive an abundance, once the stellar parameters of the object under investigation have been determined. The sensitivity of the final Li abundance to surface gravity, metallicity, and microturbulence is not very significant (see below), whereas an uncertainty of $\pm 70 \mathrm{~K}$ in $T_{\text {eff }}$ (commonly quoted as a reasonable uncertainty on this parameter, for solar-type stars) translates into \pm 0.056 dex on the final lithium abundance. Because the effective temperature is clearly the most critical parameter, it will be discussed separately, in the next section, where we provide a more detailed description of what we have learned from its derivation. Here, we will briefly comment on the other input stellar parameters and how they were determined.

The surface gravity was first determined from an inspection of the $(b-y)$ vs. $c_{1}$ diagramme (cf. Fig. 2), which allowed us to assign a preliminary $\log g$ value to each of our stars. These first-guess values were first checked versus those quoted in the literature sources used to assemble our sample. Then we finally 
Table 3. The data sample and its main characteristics, as found in the literature. The whole table is available on-line.

\begin{tabular}{rrrcrccccc}
\hline \hline HIP & HD & BD/CD & $G$ & $\begin{array}{r}V \\
\mathrm{mag}\end{array}$ & $\begin{array}{c}T_{\text {eff Lit }} \\
\mathrm{K}\end{array}$ & $\begin{array}{c}\log g_{\text {Lit }} \\
\mathrm{cm} \mathrm{s}^{-1}\end{array}$ & $\begin{array}{c}{[\mathrm{Fe} / \mathrm{H}]_{\mathrm{Lit}}} \\
\mathrm{dex}\end{array}$ & $\begin{array}{c}E W_{\text {Lit }} \\
\mathrm{mA}\end{array}$ & \begin{tabular}{c} 
Ref. $^{a}$ \\
\hline 484
\end{tabular} \\
& 97 & -206718 & & 9.660 & 5000 & $\ldots$ & -1.23 & 12.1 & 5 \\
911 & & & $266-060$ & 11.800 & 5890 & 2.2 & -1.84 & 30.8 & 3 \\
2413 & 2665 & +5670 & & 7.729 & $5050 \ldots 5100$ & 3.6 & $-1.80 \ldots-1.89$ & $15.0 \ldots 20.0$ & 5,12 \\
3026 & 3567 & $-09122 \mathrm{~B}$ & $270-023$ & 9.252 & $5858 \ldots 5930$ & 3.7 & $-1.20 \ldots-1.34$ & 45.0 & 1,4 \\
3430 & & +7131 & $242-065$ & 10.202 & $6026 \ldots 6170$ & $\ldots$ & $-1.91 \ldots-2.20$ & 31.0 & 1,4 \\
$\ldots$ & $\ldots$ & $\ldots$ & $\ldots$ & $\ldots$ & $\ldots$ & $\ldots$ & $\ldots$ & $\ldots$ & $\ldots$ \\
\hline
\end{tabular}

${ }^{a}$ The references are those given in Table 2.

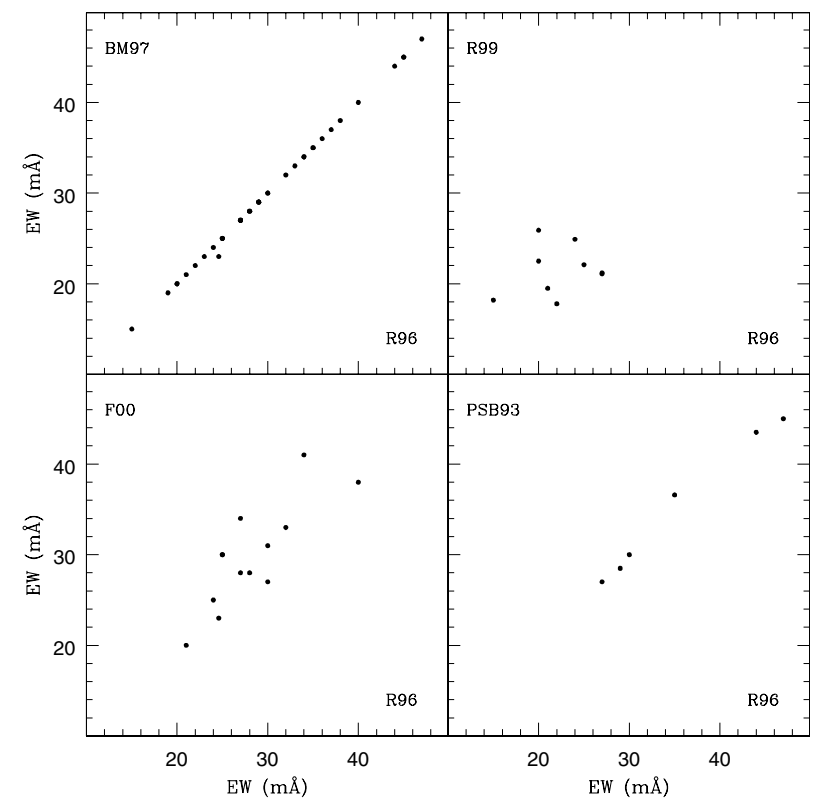

Fig. 1. The comparison of $E W \mathrm{~s}$ as measured/adopted by different authors for stars in common. The literature sources used for these comparisons are reported in the upper left and lower right corners for the $y$ - and $x$-axis respectively.

attributed to each star the $\log g$ value deduced from its position in the Herzsprung-Russel diagram (see Sect. 9.2).

In order to evaluate the sensitivity of the derived $A(\mathrm{Li})$ abundances on the stellar gravity, we performed our tests at $T_{\text {eff }}=5250 \mathrm{~K}$ and $6000 \mathrm{~K}$, for $\log g=3.0$ and 4.0 , and for two metallicities, $[\mathrm{Fe} / \mathrm{H}]=-1.0$ and -2.5 . On the average, we found that a change of 1 dex in $\log g$ affects the lithium abundance by $\simeq 0.018$ dex only, with very little dependence on the effective temperature, or on the equivalent width of the lithium line. Our findings agree very well with the common statements that the dependence of $A(\mathrm{Li})$ on the stellar gravity is negligible in the error budget (e.g. Meléndez \& Ramírez 2004).

The metallicity was first selected from the same literature sources from which we assembled our data sample. As one can see from inspecting Table 3 the agreement between different literature sources (when available) is in general quite satisfactory. Only in few cases, a large discrepancy is present but was fortunately solved because observed spectra were in hand. One such example is the star HIP 88827, for which BM97 reported

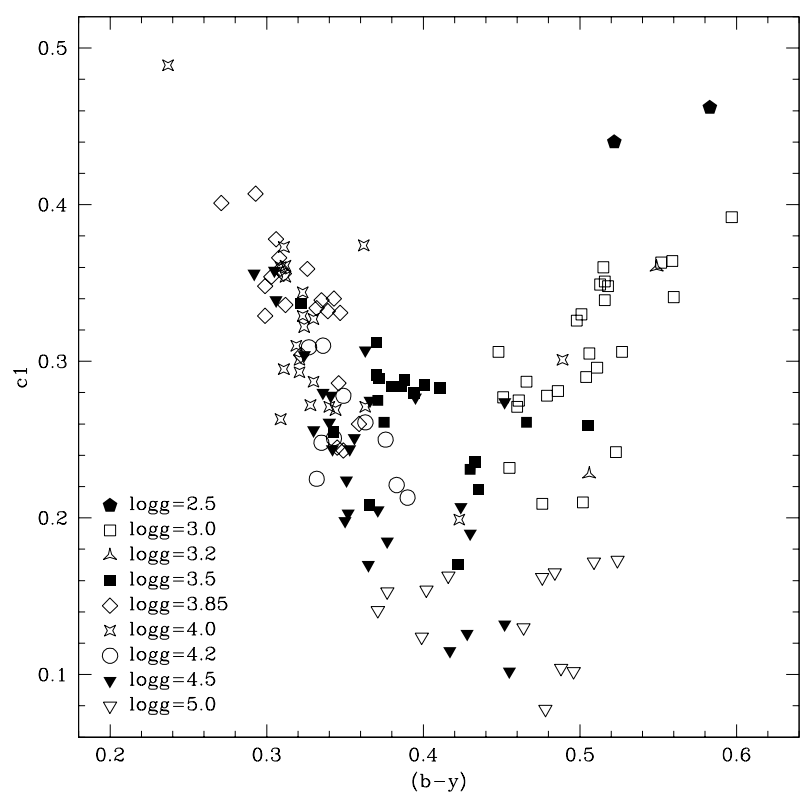

Fig. 2. The entire sample plotted in the $(b-y)$ vs. c1 diagram from which we assigned the first set of gravities. Different symbols identify different values of $\log g$, as summarised in the lower left corner of the figure.

$[\mathrm{Fe} / \mathrm{H}]=-0.91$ (taken from Alonso et al. 1996) and R96 measured -2.10: from a high resolution, high $S$ / $N$ UVES spectrum taken at the VLT, its metallicity has been recently derived from a reliable set of Fe II lines and found to be -2.4 (Nissen et al. 2002). Because of the general good agreement, our final metallicities are simply the weighted mean of all the values available for a given star (with the exception, of course, of the very few discrepant cases mentioned above). All the $[\mathrm{Fe} / \mathrm{H}]$ values determined spectroscopically from high resolution, high $S / N$ spectra were double weighted. The uncertainty on the final $[\mathrm{Fe} / \mathrm{H}]$ values is the $\sigma_{n-1}$ of the weighted mean (cf. Table 6).

We cross-checked our metallicities also photometrically, via the calibration of Schuster \& Nissen (1989, hereafter SN89 - cf. Eq. \#(3)), and taking advantage of the availability of $u b v y-\beta$ photometry for the entire sample. The satisfactory agreement we found was judged more than sufficient for our test-purposes, therefore we did not explore any further the possible systematic differences between metallicities derived spectroscopically and photometrically. 
Table 4. Photometry and reddening excesses. The whole table is available on-line.

\begin{tabular}{|c|c|c|c|c|c|c|c|c|c|c|c|c|c|c|}
\hline HIP & $(b-y)$ & $c 1$ & $m 1$ & $\beta$ & Ref. $^{a}$ & $\begin{array}{r}E(b-y) \\
\beta\end{array}$ & $\begin{array}{c}(B-V) \\
\text { Hip }\end{array}$ & $\begin{array}{c}(B-V) \\
\text { Lit }\end{array}$ & $\begin{array}{c}\mathrm{E}(B-V) \\
\mathrm{S} 98\end{array}$ & $\begin{array}{c}E(b-y) \\
\mathrm{S} 98\end{array}$ & $\begin{array}{c}E(B-V) \\
\text { H97 }\end{array}$ & $\begin{array}{c}E(b-y) \\
\text { H97 }\end{array}$ & $\begin{array}{c}E(B-V) \\
\quad \text { Lit }\end{array}$ & $\begin{array}{c}E(B-V) \\
\text { BH }\end{array}$ \\
\hline 484 & 0.513 & 0.349 & 0.155 & & 2 & & 0.787 & & 0.021 & 0.016 & $\cdots$ & $\cdots$ & & \\
\hline 911 & 0.341 & 0.278 & 0.067 & 2.582 & 1 & -0.008 & 0.570 & 0.450 & 0.020 & 0.015 & 0.022 & 0.016 & 0.000 & 0.015 \\
\hline 2413 & 0.549 & 0.360 & 0.078 & 2.730 & 2 & 0.167 & 0.792 & 0.793 & 0.395 & $\ldots$ & 0.184 & 0.134 & $\ldots$ & $\ldots$ \\
\hline 3026 & 0.332 & 0.334 & 0.087 & 2.598 & 1 & -0.002 & 0.465 & 0.460 & 0.036 & 0.026 & 0.034 & 0.025 & 0.000 & 0.015 \\
\hline 3430 & 0.309 & 0.360 & 0.040 & $\ldots$ & 2 & $\ldots$ & 0.401 & 0.390 & 0.723 & $\ldots$ & 0.034 & 0.025 & 0.000 & $\ldots$ \\
\hline$\ldots$ & $\ldots$ & $\ldots$ & $\ldots$ & $\ldots$ & $\ldots$ & $\ldots$ & $\ldots$ & $\ldots$ & $\ldots$ & $\ldots$ & $\ldots$ & $\ldots$ & $\ldots$ & $\ldots$ \\
\hline
\end{tabular}

${ }^{a}$ References: 1. Schuster \& Nissen (1988); 1a. Schuster (2002) (priv.comm.); 2. Hauck \& Mermilliod (1998); 3. Laird et al. (1988); 4. Ryan et al. (1999).

This legend refers to the table given in its entirety on-line.

Although the Li abundance is only slightly dependent on the adopted metallicity $(0.2-0.3$ dex uncertainties in $[\mathrm{Fe} / \mathrm{H}]$ affect the $\mathrm{Li}$ abundance less than $0.01 \mathrm{dex}$, cf. R96), one has to remember that a possible error in the metallicity may affect the $A(\mathrm{Li})$ vs. $[\mathrm{Fe} / \mathrm{H}]$ trend, and more importantly the $T_{\text {eff }}$ derived for that star and consequently its lithium abundance. Our calculations confirm what already found by $\mathrm{R} 99$ : if $[\mathrm{Fe} / \mathrm{H}]$ is off by 0.15 dex (a reasonable uncertainty for this parameter, especially since we have assembled our sample from a variety of analyses), the effect on $T_{\text {eff }}$ is almost negligible $(\approx \pm 20 \mathrm{~K})$ which implies an uncertainty on the $\mathrm{Li}$ abundance of less than $0.02 \mathrm{dex}$. This sensitivity applies, of course, to the parameters space spanned by our stars, with a tendency of finding larger dependences as the metallicity increases.

A similar, almost negligible dependence, is found also between Li and microturbulence, for which $\pm 0.5 \mathrm{~km} \mathrm{~s}^{-1}$ in $\xi$ correspond to \pm 0.005 dex in $A(\mathrm{Li})$. Because of this negligible dependence and after some checks of previous literature works, that included both dwarf and (sub)giant stars, we decided to run all our calculations assuming $\xi=1.5 \mathrm{~km} \mathrm{~s}^{-1}$. Because of the very small dependence of $A(\mathrm{Li})$ on microturbulence this choice gives identical results to what was implemented by PSB93, who let $\xi$ varying smoothly between 1.0 and $2.0 \mathrm{~km} \mathrm{~s}^{-1}$ going from the hotter to the cooler stars of their sample.

\section{Stellar parameters. II. The temperature scale and its weaknesses}

The main goal of any lithium analysis is to determine a fully consistent temperature scale for all the targets under examination, as the lithium abundance is strongly dependent on this stellar parameter. This approach is usually considered a guarantee of the absence of spurious differences possibly arising by having applied different criteria to the derivation of the effective temperature. Ideally, one would like to determine this parameter from first principles, i.e. to derive direct temperatures for metal-poor dwarfs. In practice, this has been achieved sofar for very few and very bright targets only (cf. RM05a, for a summary of what is currently available).

Stellar temperatures can be determined spectroscopically (e.g. via profile fitting of the wings of some of the Balmer lines, or from minimising the slope between the iron abundance - as derived from $\mathrm{Fe}$ I lines - and their excitation potential), or from photometry. Since we have assembled our data sample from the literature (i.e. no newly observed spectra), photometry is the only choice we have. Furthermore, because of the size of the sample only Strömgren uvby- $\beta$ photometry (among the photometric indices most sensitive to stellar temperatures) is available for all our stars, thanks to the extensive photometry by Schuster \& Nissen (1988), supplemented by unpublished photometry by Schuster (private communication) for approximately $10 \%$ of our stars. Table 4 (available in its entirety only on-line) summarises all the photometry we have used, together with the different colour excesses we have derived and that we will now discuss. For reference and test purposes, it also includes $(B-V)$ values taken from Hipparcos (ESA 1997) and from the literature, but we remind the reader that $(B-V)$ is not a good temperature indicator.

We derived $T_{\text {eff }}$ from the $(b-y)_{0}$ colour index using the IRFM calibrations of Alonso et al. (1996, 1999, plus the erratum from 2001) for dwarf and giant stars (cf. their Eqs. \#6 and \#14 respectively). The evolutionary status assigned to each object for the determination of the gravity (see previous section) was used to decide if a star was a dwarf or a post-main sequence object. In order to overcome the known problem of Alonso's calibration (i.e. $T_{\text {eff }}$ diverging towards high values at the lowest metallicities, cf. R99, Nissen et al. 2002), we adopted a lower limit of $[\mathrm{Fe} / \mathrm{H}]=-2.1$ in the equation. Two comments are mandatory here. Firstly, although it is important to keep in mind this a posteriori solution when discussing the findings on the most metal-poor stars of our sample, we note that Nissen et al. (2002) found no worrysome behavior (due to the assumption of this lower limit on $[\mathrm{Fe} / \mathrm{H}])$ when comparing effective temperatures derived via Alonso's colour- $T_{\text {eff }}$ calibrations from $(b-y)$ and $(V-K)$. Secondly, we note that RM05b have argued for the first time that this characteristic of the Alonso's calibration is not a problem, in the sense that it is not a numerical artifact due to the quadratic dependence on $[\mathrm{Fe} / \mathrm{H}]$ but it is intrinsic to the IRFM. In their recent work, they see a similar effect not only in the $T_{\text {eff }}$ vs. $(b-y)$ plane, but also for other colour indices. If confirmed, this would imply that adopting a lower limit on the metallicity is not justified, thus different effective temperatures would be derived. 


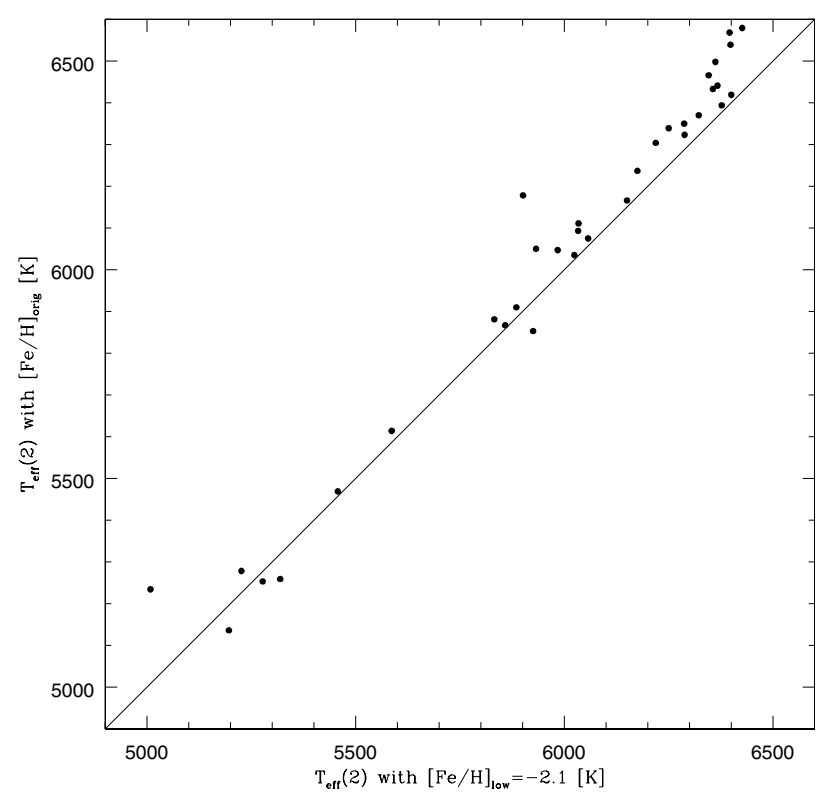

Fig. 3. A direct comparison of effective temperatures derived from the Alonso's calibration, but adopting a lower limit of $[\mathrm{Fe} / \mathrm{H}]=-2.1$ for the values plotted on the $x$-axis. Please note that $T_{\text {eff }}(2)$ refers to our final set of effective temperatures (cf. Sect. 6).

How different can be seen from Fig. 3, where we plot the effective temperatures of all our stars with $[\mathrm{Fe} / \mathrm{H}] \leq-2.1$ derived from applying or not this lower limit at -2.1 . Knowing what the dependence of $A(\mathrm{Li})$ on $T_{\text {eff }}$ is, one can already have an idea of what the effect will be on our final Li abundances. We will come back to this when discussing our results.

The interstellar reddening excess was estimated from the $(b-y)_{0}-\beta$ calibration of SN89, including a zero-point correction of $+0.005 \mathrm{mag}$ (Nissen 1994). The sample of stars from which Schuster \& Nissen (1988) derived the abovementioned relation span the metallicity range $-2.5 \leq[M / H] \leq+0.22$, and specific ranges of Strömgren colour indices, namely $2.55 \leq$ $\beta \leq 2.68,0.254 \leq(b-y) \leq 0.55,0.116 \leq c 1 \leq 0.540$, and $0.033 \leq m 1 \leq 0.470$.

However, despite $(b-y), c 1, m 1$ indices are available for all our stars, some of them fall outside the ranges of validity for the calibrating equations we have planned to use, and in few cases the $\beta$ index is missing. For consistency, one is then left with two possibilities: a) to reduce the sample to only those objects for which the complete set of valid Strömgren photometric indices is available (our sample would then be reduced to approximately 90 stars); b) to replace the missing information with other methods and carefully investigate the effects of such "pollution" (unavoidable in order to keep the number statistics high) on the final output(s). Option a) clearly represents the simplest path, and it will be used as our benchmark. Here, instead, we want to describe in some detail the series of compromises (i.e. sample pollutions) we had to introduce in order to keep working with our whole data sample. At the end, we will compare a) to b), and discuss how these two approaches affect the final Li abundances.

\subsection{The first pollution: the reddening excess without the beta index. Definition of the $\beta$-sample}

Estimating the reddening correction is probably the weakest point of any photometrically-based $T_{\text {eff }}$ scale. Interstellar reddening excesses are rarely quoted and discussed in spectroscopic analyses, despite they can affect significantly the determination of any stellar parameter, the effective temperatures in particular. In order to be consistent within the Strömgren photometry framework, we decided to use the $(b-y)_{0}-\beta$ relation to evaluate the interstellar reddening excess. However, for 24 stars ( $20 \%$ of the sample), the $\beta$ index was not found. A common solution shared by several analyses has been to assume zero reddening, based on the fact that the stars likely belong to the solar neighborhood. Alternatively, one could derive $E(b-y)$ from other colour excesses, if available. Either way, this is an approximation, that we consider as the first compromise on our data-sample. When referring to this sub-sample of stars, we will call it the $\beta$-sample.

We decided to derive $E(b-y)$ from $E(B-V)$ via the formula $E(B-V)=1.35 \times E(b-y)($ Crawford 1975), which is based on a $1 / \lambda$ reddening law and on the central wavelengths of the bandpasses. For the $E(B-V)$ colour excesses we simply averaged the $E(B-V)$ values derived from IR Dust Maps (Schlegel et al. 1998, hereafter S98) and the models of large scale visual interstellar extinction by Hakkila et al. (1997, hereafter H97). If these two sources (which will be extensively discussed in Sect. 5.4) were found to diverge significantly (e.g. in the case of HIP 49616, for which we find 0.177 from S98 maps and 0.024 from $\mathrm{H} 97$ models), that object was dropped from our list. The $E(B-V)_{\text {Lit }}$ values were given a much lower weight, being their original source not always available. However, when found in agreement with the other two $E(B-V)$ sources, they were included in the straight average. Because this solution includes a mixture of $E(B-V)$ sources, from now on we will refer to these values as $E(B-V)_{\text {mix }}$. Out of the 24 stars we have without the $\beta$ index, 14 were rescued. Figure 4 shows a direct comparison between $(b-y)_{0}$ values which have been corrected for reddening derived respectively from $\beta$ (on the $x$-axis) and from $E(B-V)_{\text {mix }}$ values and Crawford's formula (on the $y$-axis). This plot includes all the stars of our sample for which both methods could be safely applied. There is clearly some scatter around the 1:1 relation (on the order of $0.015 \mathrm{mag}$ ), and a systematic tendency of deriving larger reddenings from the indirect formula.

\subsection{The second pollution: the validity of the reddening $(b-y)_{0}-\beta$ relation. Definition of the ubvy-sample}

We mentioned above that the equation to be used in the derivation of the interstellar reddening is valid (i.e. has been tested) only for specific ranges of the Strömgren indices. Taking the photometry at face value, our sample includes a total of 35 stars, for which at least one of the Strömgren indices does not fulfill these criteria: 7 stars have the $(b-y)$ index outside the $0.254-0.550$ interval, 5 stars have the $\mathrm{c} 1$ index outside $0.116-0.540$, another 8 have the $\mathrm{m} 1$ index falling outside 


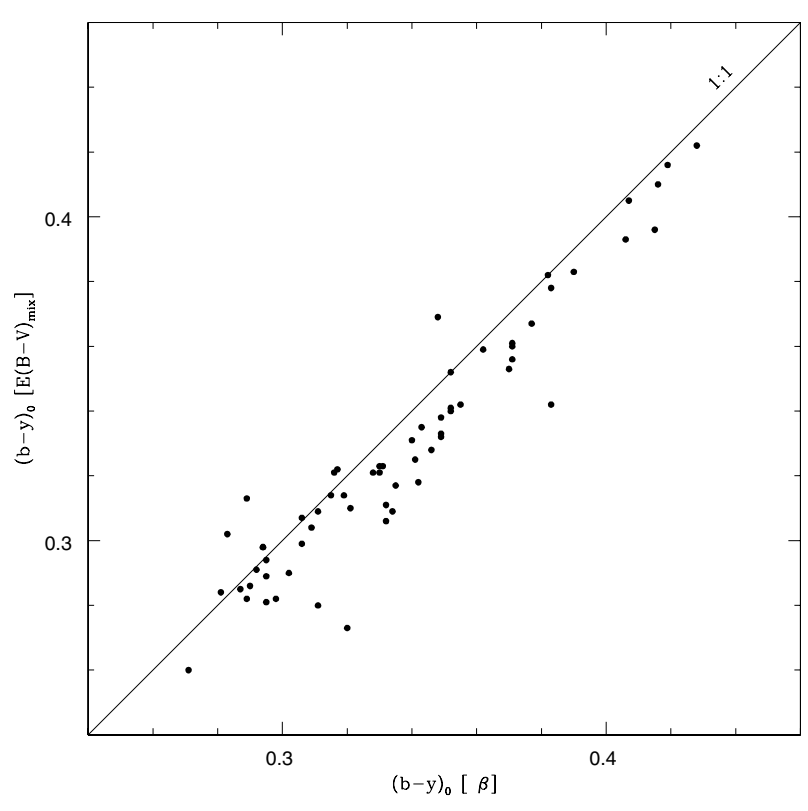

Fig. 4. A direct comparison of the $(b-y)_{0}$ index, de-reddened respectively from the $\beta$-index ( $x$-axis) and from the $E(B-V)_{\text {mix }}$ values averaged from several sources ( $y$-axis; see text for details), via Crawford's (1975) formula.

$0.033-0.470$, and 21 have the $\beta$ index outside $2.55-2.68$ (six of which also have some of the other indices off).

For the 15 (i.e., 21-6) stars with $\beta$ (only) outside the allowed range, we were able to apply the same solution described in Sect. 5.1, i.e. we derived $E(b-y)$ from $E(B-V)_{\text {mix }}$ values, to 6 of them. If the remaining 9 stars are dropped, together with those $20(7+5+8)$ that have $(b-y)$ or $\mathrm{m} 1$ or $\mathrm{cl}$ outside the allowed ranges, we are then left with a total of 111 stars, of which 20 are "polluted" because their $E(b-y)$ was derived from $E(B-V)_{\text {mix }}$ (of the initial 39=24+15 stars that belonged to this sample, 5 were dropped because one or more of their Strömgren indices fell outside the allowed ranges). We note, however, that so far we took all the photometric indices at face value, whilst each of them has its own associated uncertainty. If one were to take this into account, then the application of the validity ranges would allow some flexibility. For some stars (7 out of 20) such approach seems very reasonable: HIP 103337 , for instance, has the $(b-y)$ index off by $0.002 \mathrm{mag}$, which translates to a $10 \mathrm{~K}$ effect; all " $m 1$ " drop-outs (except two) have their photometric index off by only few thousandths of a magnitude (at most by $0.006 \mathrm{mag}$ ), which affect their final effective temperatures between $3.5 \mathrm{~K}$ and $20 \mathrm{~K}$. Because of these considerations, we decided to keep these 7 objects in a separate (polluted) sample, which we call the ubvy-sample. In summary, we are then left with a total of 118 stars.

Should we have considered also the $\beta$ values with a 3 -digits precision, six more targets would now belong to the $u b v y$-sample. Since this could influence our final discussion of the $A(\mathrm{Li})$ plateau, we will take a closer look at them once their Li abundances have been derived (cf. Sect. 7).

\subsection{The third pollution: How and when to apply the reddening corrections. Definition of the four sets of $T_{\text {eff }}$}

Knude (1979) showed that interstellar reddening is caused primarily by small dust clouds with a typical reddening of $E(b-y) \simeq 0.03$. If true, this would make any correction for reddening values smaller than 0.03 almost meaningless. This is why in the past it has been common practice to correct only those stars for which the derived excess was comparable to or larger than this value. SN89, for instance, chose $E(b-y)=0.025$ as their reference value and performed the corrections $(b-y)_{0}=(b-y)-E(b-y), c_{0}=c_{1}-$ $0.2 E(b-y)$, and $m_{0}=m_{1}+0.3 E(b-y)$ for all stars with $E(b-y) \geq 0.025$.

However, the commonly accepted picture of the nature and appearence of the interstellar reddening has significantly evolved since Knude's work and seems to suggest a patchy distribution of interstellar dust (implying that values smaller than $E(b-y)=0.03$ may be real) with a void of about 7075 pc around the Sun (e.g. Lallement et al. 2003). In principle, one should then apply the interstellar reddening excesses derived from the $(b-y)_{0}$ relation to all the stars. In practice, one has to face the problem of correcting also those stars for which negative $E(b-y)$ values are derived (28 objects in our case). This means switching to the $\beta$ index as the main $T_{\text {eff }}$ indicator, thus losing precision for those stars that are close enough to be in the void around the Sun (Nissen, priv. comm.). This explains why it is still common procedure to choose a minimum $E(b-y)$ threshold below which the colour indices are not corrected for reddening excesses. For instance, Nissen et al. (2002) have arbitrarily chosen $E(b-y)=0.015$, which corresponds to twice the sigma of $E(b-y)$.

Being unsure of what is the best approach to follow, we tested the effects of the abovementioned solutions by deriving different sets of temperatures, under the following assumptions: 1. all stars were de-reddened $\left[T_{\text {eff }}(1)\right] ; 2$. all stars were dereddened except those with negative $E(b-y)$ values [ $\left.T_{\text {eff }}(2)\right]$; 3. all stars were dereddened except those with $E(b-y) \leq 0.01$ [ $\left.T_{\text {eff }}(3)\right]$; 4. all stars were dereddened except those with negative $E(b-y)$ values and those with $E(b-y) \leq 0.01$ and $d<70 \mathrm{pc}\left[T_{\mathrm{eff}}(4)\right]$. Our four derived sets of effective temperatures are compared in Fig. 5 ( $T_{\text {eff }}(1)$ always on the $x$-axis) and show very little differences, the smallest in the case of $T_{\text {eff }}$ (1) compared to $T_{\text {eff }}(2)( \pm 27 \mathrm{~K}$ around the mean, cf. top panel). Looking at these two sets of $T_{\text {eff }}$ in more detail (the crosses in the top panel of Fig. 5 identify the 28 differing stars), one notices that for one third of these stars there is practically no difference (these are the stars for which very small reddening excesses, in absolute value, were found to be negative). Except for 3 targets, for which the difference in effective temperature is larger than $100 \mathrm{~K}$, all the others are within $\pm 50 \mathrm{~K}$, with a systematic tendency of the $T_{\text {eff }}$ (2) values to be slightly higher than $T_{\text {eff }}$ (1) ones. 


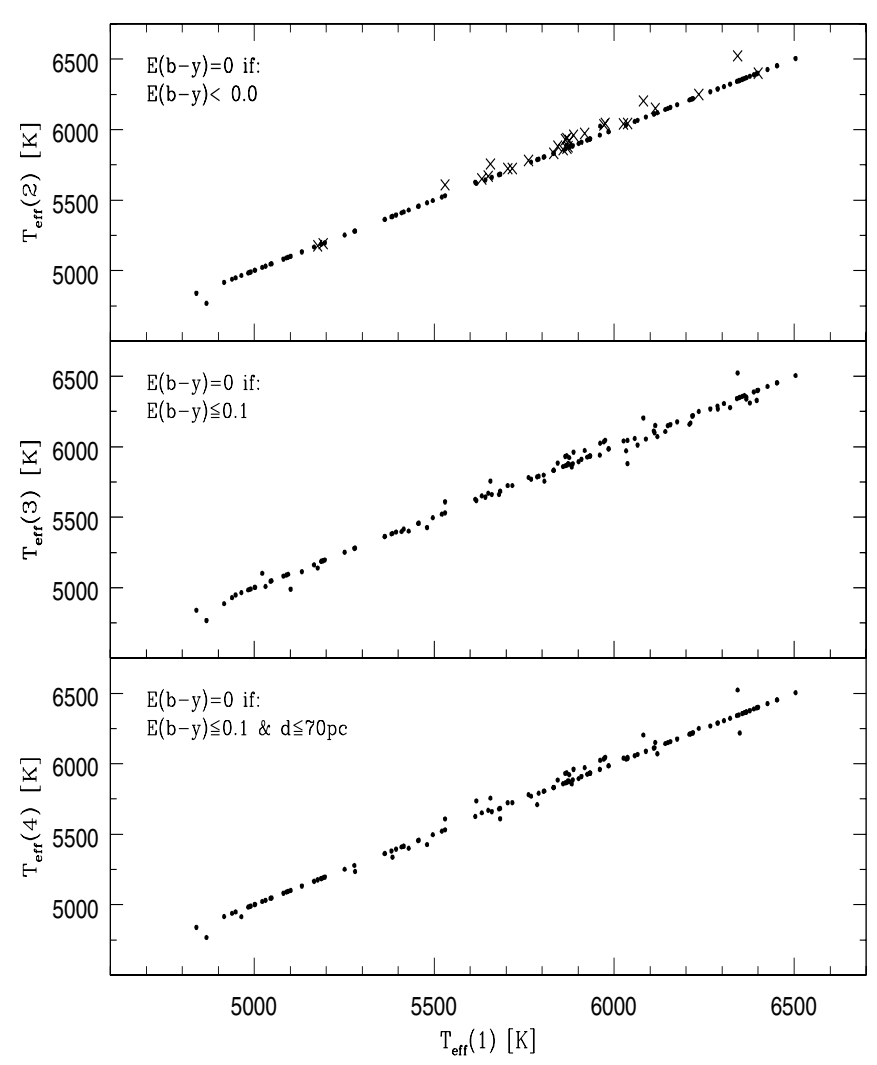

Fig. 5. How the sets of temperatures $T_{\text {eff }}$ (2), $T_{\text {eff }}$ (3), and $T_{\text {eff }}$ (4) (on the $y$-axis; see text for more explanations) compare to $T_{\text {eff }}$ (1) (on the $x$-axis). Crosses in the top panel represent the 28 objects for which the two criteria (\#1 and \#2) give different values.

\subsection{More thoughts on the interstellar reddening excess}

The fact that a very small difference in $E(b-y)$ (such as $0.005 \mathrm{mag}$, which accounts approximately for half of the common uncertainty) translates already into $35 \mathrm{~K}$ in effective temperature is a strong indication that accounting for interstellar reddening excesses plays an important role in deriving an accurate photometrically-based $T_{\text {eff }}$ scale - especially when the abundance of the element(s) under investigation is very sensitive to $T_{\text {eff }}$ like in the case of lithium. Therefore, it is important to comment also on the other sources of interstellar reddenings available in the literature.

In order to complete our comparison tests, we decided to derive reddening values also from the most recent tabulated values, i.e. those derived from infrared mapping of the dust emission distribution (Schlegel et al. 1998, S98 for short), and from the models of large scale visual interstellar extinction (Hakkila et al. 1997, hereafter H97). This was done not only to thoroughly test our photometrically-based reddening values, but especially to evaluate a posteriori the effect of mixing different sources of reddening on the derived temperature scale (a common approach to many spectroscopic abundance analyses).

Schlegel et al. (1998) estimated the dust column densities from the COBE/DIRBE and IRAS/SISSA infrared maps of dust emission over the entire sky, and transformed them to reddenings by using colours of elliptical galaxies. In other words, these maps give reddening values as if the objects lie outside the Galaxy, hence they may overestimate the real reddening, especially for relatively nearby objects. Also, at low latitudes $(|b|<5 \mathrm{deg})$ the removal of IR point sources is not optimal, hence the derived reddening values may be strongly affected. The quoted errors are of the order of $16 \%$.

Hakkila et al. (1997) instead, developed a numerical algorithm to model the large scale Galactic clumpy distribution of obscured interstellar gas and dust by using published results of large-scale visual interstellar extinction. It is concentrated towards the Galactic plane and it varies as a function of Galactic longitude and latitude. These estimates depend on the assumed distance, which is one of the input parameters to the algorithm. A word of caution concerns its inability in identifying small scale (less than $1 \mathrm{deg}$ ) extinction variations, and the fact that reddening estimates for mid-Galactic latitudes (7 deg $\leq b \leq 16 \mathrm{deg}$ ) and for distances between 1 and $5 \mathrm{kpc}$ in the Galactic plane are more unsecure. The quoted errors are not very meaningful since they represent the mean of the errors as reported in the original studies, hence they are likely overestimated.

Table 4 presents an overview on how reddening excesses derived from different methods compare to each other. Columns 2 to 7 report Strömgren photometry and $E(b-y)_{\beta}$ values as derived from the $(b-y)_{0}-\beta$ calibration (see previous sub-sections), while Cols. 8 and 9 list the Hipparcos- and literature-based $(B-V)$ values. Column 10 reports the $E(B-V)$ values as derived from the $\mathrm{S} 98$ maps, while Col. 12 lists the values as derived from the H97 algorithm. The corresponding $E(b-y)$, derived via the Crawford (1975) relation are reported in Cols. 11 and 13 respectively.

Two remarks are important. First, some very high values of $E(B-V)$ are derived from the S98 maps (by using the IDL code made available by the same authors), which look unrealistic when compared to all the other $E(B-V)$ values. Since the main purpose of our tests is to have some feeling on the possible scatter introduced by mixing reddening values taken from different sources, without being biased by outliers, we did not investigate these high values any further. Hence, they have been discarded from all comparison figures and tests. However, one can easily identify them in Table 4 , since no corresponding $E(b-y)$ value was derived (same applies also to Hakkila-based $E(B-V)$ values - though for significantly fewer stars). One should also note that in Table 4 there remain some suspiciously high $E(B-V)$ values.

Secondly, in order to survey as many choices of reddening as possible, Table 4 includes also $E(B-V)$ values as found in the literature sources from which we assembled our data sample (Col. 14 labeled $\left.E(B-V)_{\text {Lit }}\right)$ and as derived from the neutral hydrogen $\mathrm{H}$ I column density distribution of Burstein \& Heiles (1982, BH for short - Col. 15 labeled $\left.E(B-V)_{\mathrm{BH}}\right)$ in correlation with deep galaxy counts. A partial summary of Table 4 is provided in Fig. 6, where two comparisons are plotted simultaneously: the filled circles show the relation between $E(B-V)$ values derived from the $\mathrm{S} 98$ (on the $x$-axis) and from the $\mathrm{BH}$ maps (on the $y$-axis). The crosses represent the comparison between $E(B-V)$ values derived from the S98 IR dust maps (on the $x$-axis) and from the H97 model (on the $y$-axis). The 1:1 


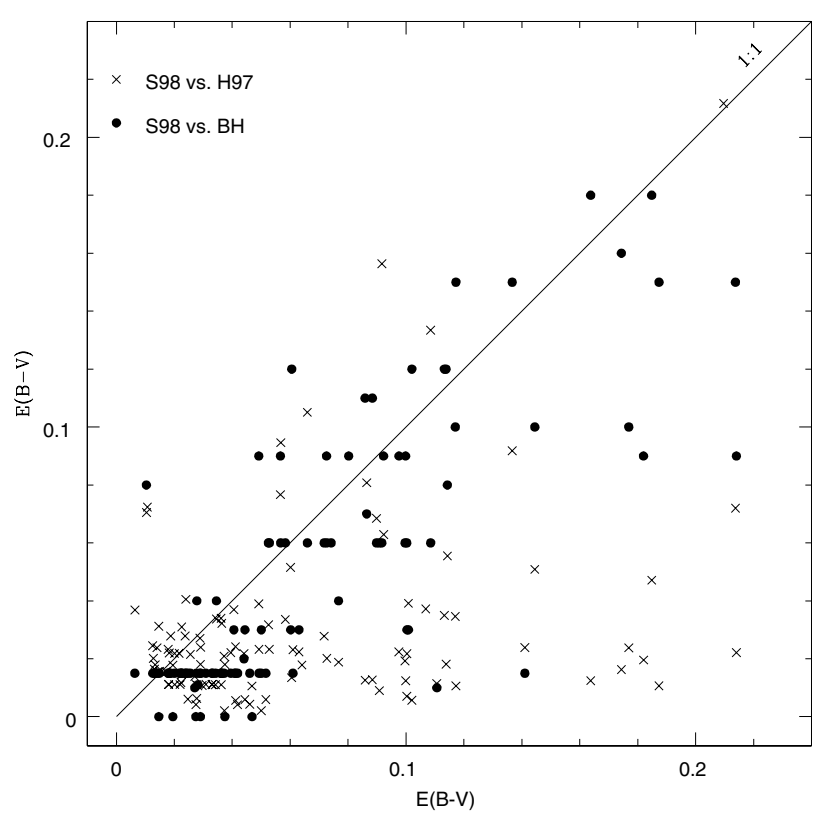

Fig. 6. Comparison between $E(B-V)$ values derived from different methods: filled dots show how $E(B-V)$ colour excess compare when derived from the InfraRed Dust Maps of Schlegel et al. (1998, on the $x$-axis) and of Burstein \& Heiles (1982, on the $y$-axis); crosses represent a similar comparison between $E(B-V)_{\mathrm{S} 98}$ and $E(B-V)_{\mathrm{H} 97}$ (on the $x$ - and $y$-axis respectively).

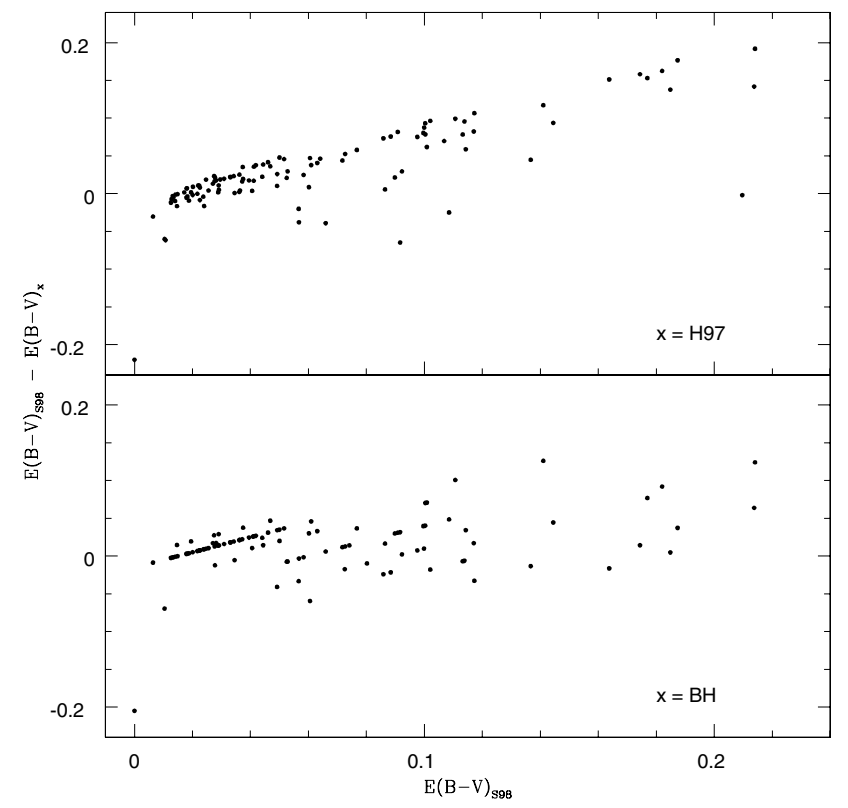

Fig. 7. Comparison between $E(B-V)_{\mathrm{S} 98}$ (on the $x$-axis) and differences between $E(B-V)$ values derived from various sources (identified from the acronym given in the right bottom corner of each pan), always using $\mathrm{S} 98$ as the reference.

relation is plotted for comparison. From this figure, one immediately notices the lack of a tight correlation between the $E(B-V)$ values derived from the maps of Schlegel et al. and Burstein \& Heiles. The comparison with H97 clearly shows that Hakkila's model of the Galactic interstellar dust distribution tends to give reddening values much lower than the S98 IRDM. Another way to look at these comparisons is by

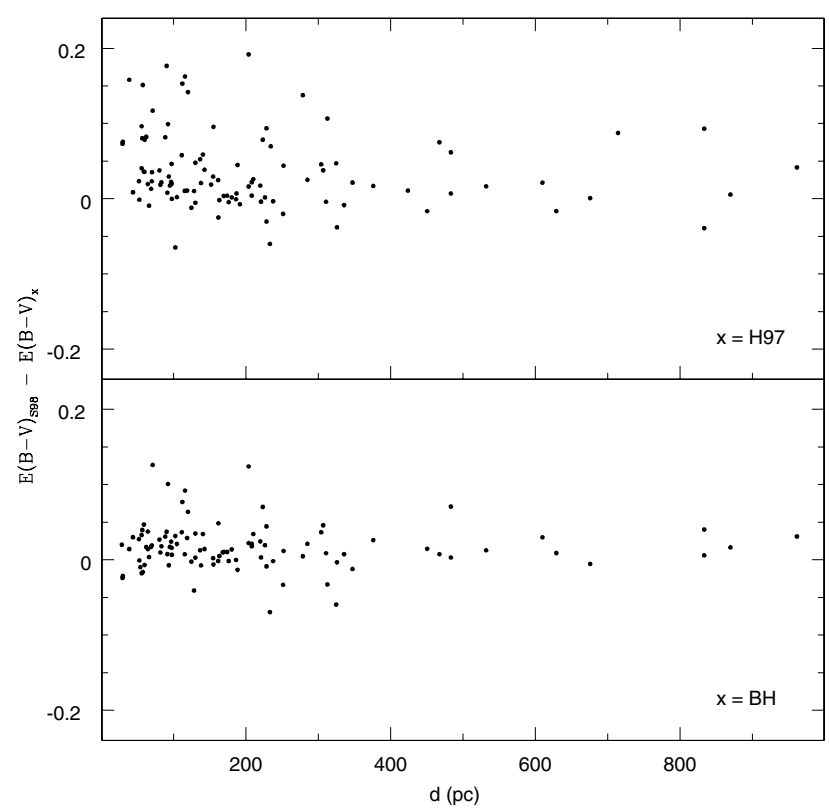

Fig. 8. Comparison between $E(B-V)$ values derived from various sources (identified from the acronym given in the right bottom corner of each pan) and the distance of the object, as derived from Hipparcos parallaxes.

inspecting Fig. 7, where the difference between the different reddening estimates (always with respect to S98 values) are plotted versus $E(B-V)_{\mathrm{S} 98}$. From this figure it becomes clear that there are systematic differences between S98 reddening values and the other two estimates, although there does not seem to be any dependence on the distance, except for a slightly larger dispersion at small distances (cf. Fig. 8).

In summary, based on the abovementioned arguments, it is very hard to defend the position that we know reddening better than $0.007-0.010 \mathrm{mag}(2 \sigma)$ which correspond already to affecting the temperature determination by $50-70 \mathrm{~K}$ (and in turn the lithium abundance by $\sim 0.05 \mathrm{dex}$ ).

\section{Our final choice: temperature and data-sample(s)}

Because lithium abundances are mostly sensitive to the choice of the stellar temperature, our main goal has so far focused on how to derive a temperature scale as consistent as possible. In order to do that, we chose to derive photometric temperatures since our sample has been assembled from different literature sources. Figure 9 shows how well our final set of $T_{\text {eff }}$ correlates with $(b-y)_{0}$ (i.e., the Strömgren index our temperature scale is based on). During this process (cf. Sect. 5) we faced some of the major drawbacks of such determinations, namely reddening and applicability of colour- $T_{\text {eff }}$ relations. These are summarised in Figs. 3-8. By inspecting Fig. 5 and noticing how small the differences between $T_{\text {eff }}$ (1) and $T_{\text {eff }}$ (2) are, we have selected $T_{\text {eff }}(2)$ as our final set of effective temperatures (listed in the second column of Table 5, labeled \#2). As a reminder, this was derived by de-reddening only those stars for which $E(b-y)$ was found to be positive. 


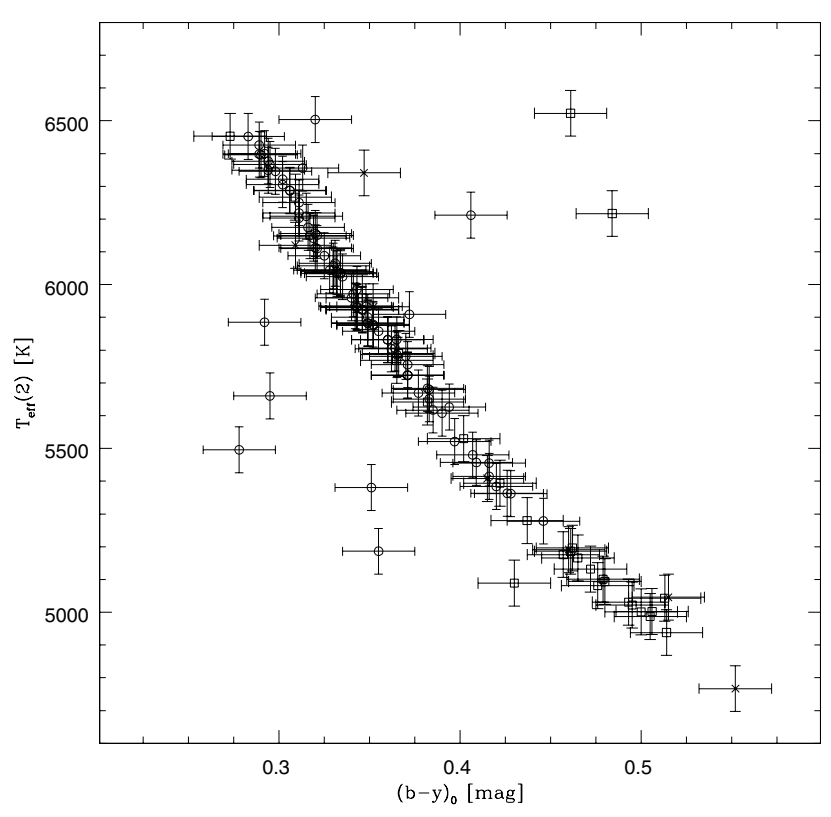

Fig. 9. How our final set of temperatures $T_{\text {eff }}$ (2) correlates with the de-reddened Strömgren index $(b-y)_{0}$, demonstrating the strong sensitivity of the index to effective temperature.

Table $5^{5}$ summarises all sets of temperatures that have resulted from our several digressions: Cols. 2 to 5 report the effective temperatures derived by applying the reddening $(b-$ $y)_{0}-\beta$ relation and making different assumptions about it (cf. Sect. 5.3), whereas Cols. 6 and 7 represent sets of temperatures as derived by using different sources of reddening. The latter two columns are useful comparisons to check how much scatter and slope on the $A(\mathrm{Li})$-plateau could originate just by mixing different reddening sources in the same analysis. Figure 10 clearly shows how different the derived temperatures can be when the reddenings are derived from different methods: if they are taken from the IRDM of S98, for instance, the derived temperatures are significantly higher $(+178 \mathrm{~K}$ on the average, with a dispersion around the mean of $\pm 236 \mathrm{~K}$ ). Of course, depending on for which targets one may need to select a different source of reddening excess, there may well be some artificial scatter emerging among their $\mathrm{Li}$ abundances.

Another relevant comparison to make would be the one between our $T_{\text {eff }}$ scale and the $T_{\text {eff }}$ values used in the original literature sources from which our list of targets was assembled. We present these comparisons in Fig. 11, in the form of $T_{\text {eff }}(2)-T_{\text {eff }}(\mathrm{X})$ vs. $T_{\text {eff }}(2)$, where $T_{\text {eff }}(2)$ is our preferred and finally selected set of temperatures and $T_{\text {eff }}(\mathrm{X})$ represents the temperature scales used in the original works from which our list of targets was assembled (cf. Table 2). Here, we selected to plot those literature analyses from which we took the largest numbers of stars, except for the bottom panel where the comparison with the very recent RM05a $T_{\text {eff }}$ scale is presented. We remind the reader that our list of targets does not include objects from this work because the analyses were carried out almost simultaneously. As one can see, our temperature scale $\left(T_{\text {eff }}(2)\right)$ tend to be always higher, the largest difference

\footnotetext{
5 Only available in its entirety on-line.
}

Table 5. Final temperatures as derived from different reddening corrections. The whole table is available on-line.

\begin{tabular}{|c|c|c|c|c|c|c|}
\hline \multirow[t]{2}{*}{ HIP } & \multicolumn{6}{|c|}{ Effective temperature scales (K) } \\
\hline & 2 & 1 & 3 & 4 & S98 & H97 \\
\hline \multicolumn{7}{|c|}{ Sample \#1: the clean sample } \\
\hline 911 & 5972 & 5918 & 5972 & 5972 & 6076 & 6065 \\
\hline 3026 & 6040 & 6026 & 6040 & 6040 & 6221 & 6214 \\
\hline 3446 & 5901 & 5901 & 5894 & 5901 & 5991 & 5995 \\
\hline 3564 & 5683 & 5683 & 5683 & 5683 & 5974 & 5679 \\
\hline 8572 & 6287 & 6287 & 6287 & 6287 & 6265 & 6287 \\
\hline$\cdots$ & $\cdots$ & $\cdots$ & $\cdots$ & $\cdots$ & $\cdots$ & .. \\
\hline \multicolumn{7}{|c|}{ Sample \#2: the $\beta$ sample } \\
\hline 484 & 5064 & 5064 & 5064 & 5064 & 5064 & \\
\hline 3554 & 5008 & 5008 & 5008 & 5008 & 5040 & 5022 \\
\hline 4343 & 5064 & 5064 & 5064 & 5064 & 5069 & 5064 \\
\hline 8314 & 6430 & 6430 & 6430 & 6430 & $\cdots$ & 6444 \\
\hline 13749 & 4965 & 4965 & 4965 & 4965 & 4996 & 4965 \\
\hline$\cdots$ & $\ldots$ & $\cdots$ & $\ldots$ & $\ldots$ & $\ldots$ & $\ldots$ \\
\hline \multicolumn{7}{|c|}{ Sample \#3: the $u b v y$ sample } \\
\hline 12807 & 5932 & 5932 & 5932 & 5932 & 6872: & 5959 \\
\hline 83320 & 5984 & 5984 & 5984 & 5984 & 6251 & 5861 \\
\hline 87062 & 5909 & 5909 & 5909 & 5909 & $\ldots$ & 5905 \\
\hline 91129 & 6217 & 6217 & 6217 & 6217 & 7081: & 6237 \\
\hline 103337 & 4885 & 4885 & 4885 & 4885 & 4876 & 4876 \\
\hline$\ldots$ & $\ldots$ & $\ldots$ & $\ldots$ & $\ldots$ & $\cdots$ & $\cdots$ \\
\hline
\end{tabular}

being with PSB93 (on average $+158 \mathrm{~K}$, with a dispersion around the mean of $\pm 136 \mathrm{~K})$. The smallest differences, on average, are between us and BM97 (+6 K) and RM05a (practically zero), but the dispersions around these means remain on the order of $\pm 100-150 \mathrm{~K}$.

Also, in Table 5 all our targets are grouped in three different sub-samples. As stated at the beginning of Sect. 5, in order to be fully consistent with the analytical method one chooses to follow, one is usually forced to work with a much smaller sample of stars compared to the initial data-set: in our case, 91 stars compared to the original 146. In order to avoid this drastic reduction, we explored alternative solutions which allowed us to retain a larger number of objects (118), but at the price of contaminating part of the sample as follows:

Sample \#1 is the clean sample: it includes 91 stars, for which the complete set of Strömgren photometric indices are available, and for which the SN89 calibrations can be successfully applied.

Sample \#2 is the $\beta$ sample: it includes 20 stars, for which the reddening value $E(b-y)$ was derived from averaging different sources of $E(B-V)$ values (S98, H97, and literature cf. Sect. 5.1 for details on how this average was performed), via Crawford's formula (1975). We note that no correction was applied to these stars to compensate for the offset seen in Fig. 4.

Sample \#3 is the $u b v y$ sample: it includes 7 stars, for which one of the $u b v y$ photometric indices $(b-y, c 1, m 1)$ falls just 


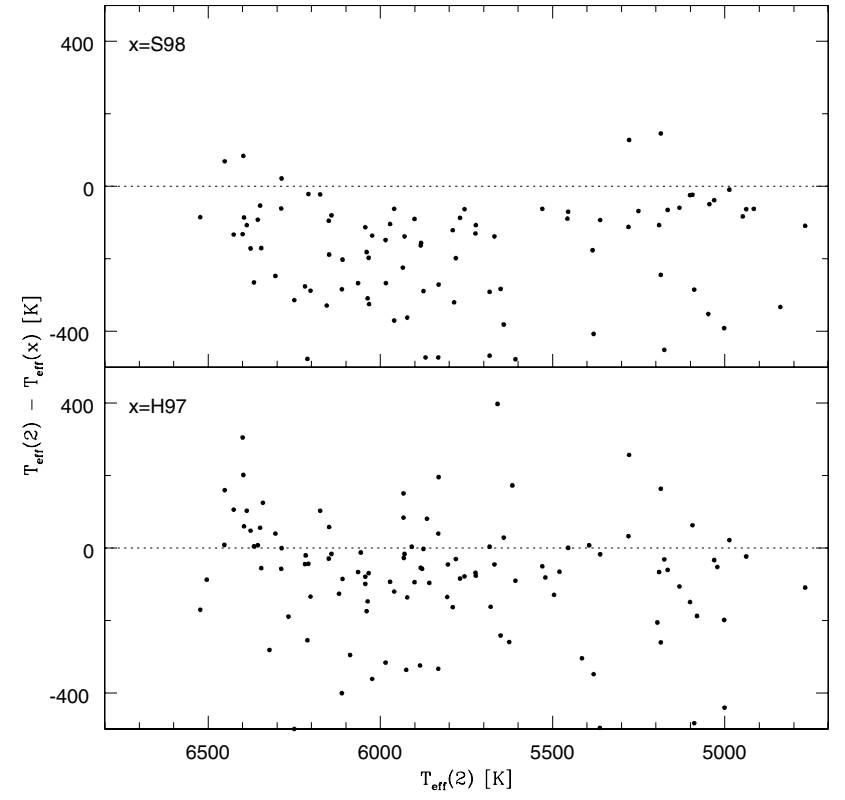

Fig. 10. Comparison between our final set of effective temperatures $T_{\text {eff }}(2)$ (on the $x$-axis) and the temperatures computed by using $E(b-y)$ as derived from $E(B-V)_{\mathrm{S} 98}$ and $E(B-V)_{\mathrm{H} 97}$ (on the $y$-axis, from top to bottom).

slightly outside (see last paragraph of Sect. 5.2) the allowed intervals for the application of the Nissen \& Schuster (1989) calibrations.

\section{The lithium abundance}

The final assessment of how relevant and important all our tests have been can be made only after comparing the lithium abundances derived from the different sets of temperatures and for the different sub-samples.

The lithium abundance for all the stars of our sample was determined from the equivalent widths $(E W \mathrm{~s})$ of the $670.7 \mathrm{~nm}$ line as reported in the literature works from which we assembled the data sample. Table $6^{6}$ lists the mean equivalent width and its $1 \sigma$ uncertainty that were used in our computations of the $\mathrm{Li}$ abundance: we opted for the mean value because of a satisfactory overall agreement found in the literature (cf. Table 3, for the corresponding references, listed in the last column of the table).

The lithium abundance was derived under the assumption of Local Thermodynamic Equilibrium (LTE) using Kurucz (1993) WIDTH9 and model atmospheres with the overshooting option switched off (cf. Castelli et al. 1997 for the models, and Molaro et al. 1995 for comparisons between different versions of Kurucz model atmospheres). The $g f$-value we used for the $\mathrm{Li} \mathrm{I}$ is 0.171 , for which the VALD database reports an accuracy of $3 \%$.

As Carlsson et al. (1994) have shown, the LTE approximation when deriving $\mathrm{Li}$ abundances for cool stars is not a realistic representation of the physics present in the atmospheric layers where the $670.7 \mathrm{~nm}$ line forms. Since non-LTE corrections

\footnotetext{
${ }^{6}$ Available in its entirety on-line.
}

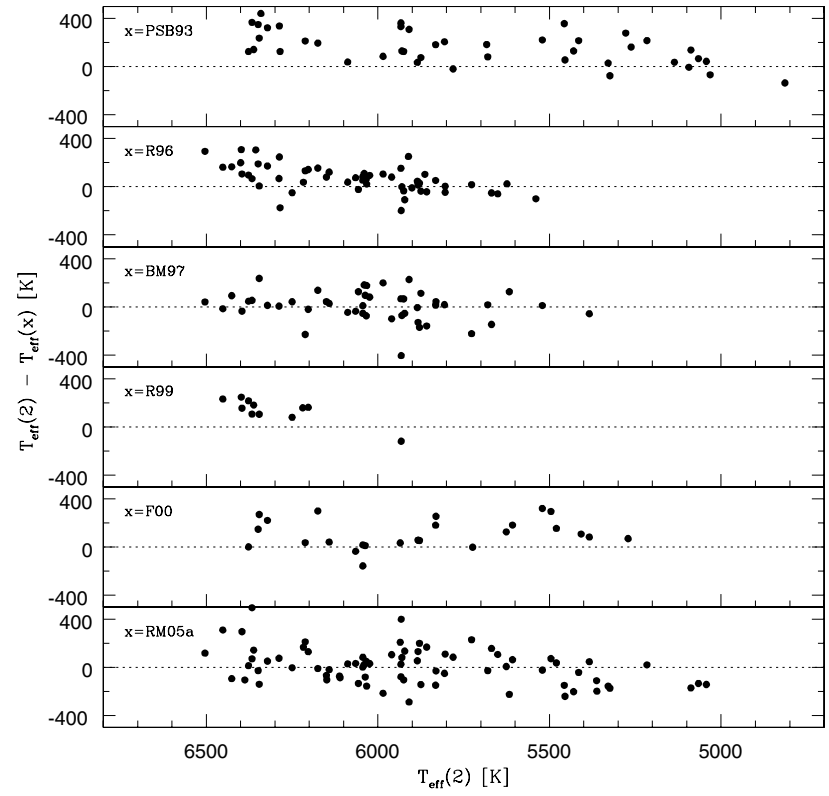

Fig. 11. Comparison between our final set of effective temperatures $T_{\text {eff }}$ (2) (on the $x$-axis) and the temperature scales used/derived in the original literature sources (on the $y$-axis). Acromyms in the upper left corner identify which analysis is used for the comparison.

vary in sign and size when spanning a large range of stellar parameters (being larger for cooler stars), ignoring these corrections may clearly affect any interpretation of the $A(\mathrm{Li})$-plateau and its possible slope with effective temperature and metallicity. Therefore, NLTE corrections were computed with the interpolation code made available by Carlsson et al. (1994) and applied to our LTE $A(\mathrm{Li})$ abundances. We note that no NLTE correction could be derived for those stars with $A(\mathrm{Li})_{\mathrm{LTE}}$ abundances smaller than 0.6 , because the interpolation code works on a given range of input parameters (for instance, in the case of the $\mathrm{Li}$ abundance, the range is $A(\mathrm{Li})=0.6-4.2)$. Furthermore, despite these ranges of input parameters, not all the combinations are covered in the table which contains the tabulated coefficients from which the NLTE corrections are computed. In our sample, we had only few of these cases, namely two stars had a metallicity lower than the minimum threshold (-3.0), and another couple of stars had their effective temperatures slightly higher of the maximum threshold (by 4 and $23 \mathrm{~K}$ respectively). For these objects, we rounded off their parameters to the nearest allowed value, and computed the NLTE correction.

The resulting LTE Li abundances (both LTE and NLTE are given only for our final set of $T_{\text {eff }}$ ) are presented in Table 6 , where we give also other relevant parameters like the metallicity and its $1 \sigma$ uncertainty as derived from a critical analysis of the literature. Three different sets of lithium abundances are reported for each target, depending on which set of $T_{\text {eff }}$ was used (cf. Table 5). For the $A(\mathrm{Li})$ values (which were derived from our final set of temperatures, i.e. $\left.T_{\text {eff }}(2)\right)$ we also give the associated $\pm 1 \sigma(E W)$ error (Cols. 9, 10). Similar uncertainties apply also to the lithium abundances listed under the last two columns of the table. 
Table 6. Final Li abundances as derived by using different sets of $T_{\text {eff }}$. The whole table is available on-line.

\begin{tabular}{|c|c|c|c|c|c|c|c|c|c|c|c|c|}
\hline "HIP & bin & $\begin{array}{c}\overline{[F e / H]}] \\
\operatorname{dex}\end{array}$ & $\begin{array}{c}\sigma(\mathrm{Fe}) \\
\operatorname{dex}\end{array}$ & $\begin{array}{c}\log g \\
\mathrm{~cm} \mathrm{~s}^{-1}\end{array}$ & $\begin{array}{l}E W \\
\mathrm{~mA}\end{array}$ & $\begin{array}{l} \pm 1 \sigma \\
\mathrm{mA}\end{array}$ & $\begin{array}{c}A(\mathrm{Li})_{\text {LTE }} \\
\operatorname{dex}\end{array}$ & $\begin{array}{c}A(\mathrm{Li})_{\text {NLTE }} \\
\text { dex }\end{array}$ & $\begin{array}{l}+1 \sigma \\
\operatorname{dex}\end{array}$ & $\begin{array}{c}-1 \sigma \\
\operatorname{dex}\end{array}$ & $\begin{array}{c}A(\mathrm{Li})_{\mathrm{S} 98} \\
\text { dex }\end{array}$ & $\begin{array}{c}A(\mathrm{Li})_{\mathrm{H} 97} \\
\text { dex }\end{array}$ \\
\hline \multicolumn{13}{|c|}{ Sample \#1: the clean sample } \\
\hline 911 & & -1.84 & 0.15 & 4.50 & 30.8 & 3.5 & 2.117 & 2.112 & 0.042 & 0.067 & 2.200 & 2.191 \\
\hline 3026 & & -1.25 & 0.07 & 3.85 & 45.0 & 6.0 & 2.428 & 2.418 & 0.078 & 0.086 & 2.573 & 2.567 \\
\hline 3446 & & -3.50 & 0.10 & 4.50 & 27.0 & 3.9 & 1.973 & 1.986 & 0.074 & 0.076 & 2.045 & 2.048 \\
\hline 3564 & & -1.27 & 0.15 & 3.50 & 35.2 & 3.5 & 2.011 & 2.054 & 0.056 & 0.076 & 2.244 & 2.008 \\
\hline 8572 & & -2.51 & 0.01 & 3.85 & 27.0 & 1.4 & 2.257 & 2.236 & 0.026 & 0.027 & 2.239 & 2.257 \\
\hline$\ldots$ & & $\ldots$ & $\ldots$ & $\ldots$ & $\ldots$ & $\ldots$ & $\ldots$ & $\ldots$ & $\ldots$ & $\ldots$ & $\ldots$ & $\ldots$ \\
\hline
\end{tabular}

\begin{tabular}{rcccccccccccc}
\hline \multicolumn{10}{c}{ Sample \#2: the $\beta$ sample } \\
\hline 484 & -1.23 & 0.15 & 3.00 & 12.1 & 3.5 & 0.953 & 1.101 & $\ldots$ & $\ldots$ & 0.949 & $\ldots$ \\
3554 & -2.87 & 0.15 & 3.00 & 17.7 & 3.5 & 1.018 & 1.153 & 0.087 & 0.104 & 1.044 & 1.238 \\
4343 & -2.08 & 0.15 & 3.00 & 9.1 & 3.5 & 0.766 & 0.912 & 0.150 & 0.219 & 0.878 & 0.680 \\
8314 & $?$ & -1.68 & 0.09 & 4.00 & 27.0 & 3.0 & 2.378 & 2.337 & 0.053 & 0.058 & 2.262 & 2.523 \\
13749 & -1.62 & 0.15 & 3.00 & 14.6 & 3.5 & 0.876 & 1.039 & 0.102 & 0.128 & 0.901 & 0.870 \\
$\ldots$ & $\ldots$ & $\ldots$ & $\ldots$ & $\ldots$ & $\ldots$ & $\ldots$ & $\ldots$ & $\ldots$ & $\ldots$ & $\ldots$ & $\ldots$ \\
\hline
\end{tabular}

\begin{tabular}{rcccccccccccc}
\hline \multicolumn{10}{c}{ Sample \#3: the $u b v y$ sample } \\
\hline 12807 & -2.87 & 0.22 & 4.50 & 22.9 & 3.0 & 1.918 & 1.929 & 0.066 & 0.068 & 2.676 & 1.940 \\
83320 & -2.56 & 0.15 & 3.50 & $<5.0$ & $\ldots$ & $<1.265$ & $<1.287$ & $\ldots$ & $\ldots$ & $<1.603$ & 1.167 \\
87062 & -1.67 & 0.23 & 4.50 & 31.5 & 4.0 & 2.109 & 2.111 & 0.088 & 0.069 & 2.109 & 2.106 \\
91129 & $*$ & -2.96 & 0.10 & 4.50 & 27.3 & 2.5 & 2.208 & 2.191 & 0.045 & 0.054 & 2.899 & 2.224 \\
103337 & -2.07 & 0.15 & 3.00 & 25.8 & 3.5 & 1.025 & 1.197 & 0.069 & 0.072 & 1.179 & 0.877 \\
$\ldots$ & $\ldots$ & $\ldots$ & $\ldots$ & $\ldots$ & $\ldots$ & $\ldots$ & $\ldots$ & $\ldots$ & $\ldots$ & $\ldots$ & $\ldots$ \\
\hline
\end{tabular}

? Identifies a suspected binary (Latham et al. 2002; Carney et al. 1994, 2003).

* Identifies a confirmed single- or double-lined binary (from Latham et al. 2002; Carney et al. 1994, 2003).

Note that the complete version of the table (available only on-line) reports the complete legend of symbols and references.

\subsection{What is the best achievable accuracy?}

The accuracy of any abundance determination mainly depends on the following factors: the quality of the observational sample (for the continuum placement and the measurement of equivalent widths), the choice of the stellar parameters characterising each star of the analysed sample, the atomic physics (e.g. the oscillator strength of the transition(s) under investigation), and the analytical tools that have been used (e.g. model atmospheres).

Our sample was assembled from the literature, following some selection criteria on the quality of the analyses, i.e. high resolution and high $S / N$. Since the $\mathrm{Li} I$ line falls in a very clean spectral region, with very few neighboring absorption lines, the placement of the continuum is usually quite accurate (on the order of $1-2 \%$ ) if the data quality is high. This uncertainty is usually included in the uncertainty associated to the equivalent width measurement.

For the latter, because of the generally quite satisfactory agreement between different literature sources (on a given target, cf. Fig. 1) we decided to use as our final $E W$ the arithmetic mean of all the measurements, and take the dispersion around the mean as the uncertainty on each measurement. When only one measurement was available, the associated uncertainty is the error quoted in the original work. Table 6 reports both the error on each $E W$ and the corresponding $1 \sigma$ uncertainty on the $\mathrm{Li}$ abundance. Except for few cases, the latter are well below 0.1 dex. The uncertainty due to a $\pm 3 \%$ error in the $\log g f$ value is $\pm 0.013 \mathrm{dex}$.

Lithium abundances are known to be very sensitive to the chosen effective temperature, but their dependence on the other parameters, i.e. gravity, metallicity, and microturbulence is negligible. Common uncertainties on $\log g,[\mathrm{Fe} / \mathrm{H}]$ and $\xi$ $\left( \pm 0.25 \mathrm{dex}, \pm 0.15 \mathrm{dex}\right.$, and $\pm 0.3 \mathrm{~km} \mathrm{~s}^{-1}$ respectively) affect the final Li abundances by at most $0.005 \mathrm{dex}, 0.015 \mathrm{dex}$, and 0.003 dex. When summed under quadrature, the resulting uncertainty is around 0.017 dex only.

On the contrary, the dependence of $\mathrm{Li}$ abundances on the effective temperature is much stronger. An uncertainty of $\pm 70 \mathrm{~K}$ in $T_{\text {eff }}$ (commonly quoted as a reasonable uncertainty on this parameter) translates into a \pm 0.05 dex on the lithium abundance. For this work, we have considered only the uncertainties associated to the photometric indices $(b-y)$ (generally quoted to be around $0.008 \mathrm{mag}$, cf. Nissen et al. 2002) and $\beta$ from which we have derived our reddening estimates (generally quoted to be around $0.011 \mathrm{mag}$ ). When summed under quadrature, this gives us an average uncertainty on our effective temperatures of $\pm 75 \mathrm{~K}$, which corresponds to $\pm 0.054 \mathrm{dex}$ in $A(\mathrm{Li})$. 
Combining all the uncertainties together, we find that depending on the $\pm 1 \sigma(E W)$ error on $A(\mathrm{Li})$ our best achievable accuracy is 0.06 dex. In the worst cases it could be as high as $0.15 \mathrm{dex}$, but one should notice that for all the stars for which a $\mathrm{Li}$ abundance uncertainty larger than 0.1 dex has been derived, the equivalent width of the $\mathrm{Li}$ I line is always quite small (in the 5-15 mÅrange) with a very significant $1 \sigma E W$ quoted error. Although we do not have the observed spectra available for further checking, this indicates that $S / N$ ratios on the order of 100 are probably too low for accurate measurements of weak $\mathrm{Li}$ lines. If one were to exclude those stars with very small equivalent widths (and large quoted uncertainties) then our final (individual) accuracies range between 0.06 and $0.1 \mathrm{dex}$.

Last but not least, one should not forget that our abundances were derived based on Kurucz non-overshooting model atmospheres and that most of the current Li analyses are carried out under LTE assumptions (with NLTE corrections applied to them) and with one-dimensional model atmospheres. Choosing a different treatment of the convective motions in the model atmospheres (i.e. choosing the so-called Kurucz overshooting models) has the effect of deriving slightly higher $\mathrm{Li}$ abundances (by $\simeq 0.08 \mathrm{dex}$ ) but both sets of models carry similar uncertainties which are difficult to quantitatively assess. NLTE corrections also carry their own uncertainty, but this is small and well within the average abundance errors, according to Carlsson et al. (1994). Finally, although $\mathrm{Li}$ abundances determined using 3D-hydrodynamical model atmospheres and corrected for NLTE effects differ from 1D NLTE Li abundances by less than 0.1 dex ( 0.05 dex for the few stars that have been investigated so far, cf. Asplund et al. 2003; and Barklem et al. 2003), these same authors warn about possible dependences on temperature and metallicity, that could clearly affect any discussion on the existence or lack thereof of a slope in the $A(\mathrm{Li})$-plateau with $T_{\text {eff }}$ and/or metallicity. Hence, no conclusion can be final, until 3D NLTE effects on Li abundances are mapped on a larger stellar parameters space.

\subsection{More on the accuracy issue}

Since one of the main focuses of this work is a critical assessment of how accurately Li abundances in halo stars can be determined and the Li plateau can be characterised (via its width, spread and slopes with $T_{\text {eff }}$ and metallicity), comparisons between our derived $\mathrm{Li}$ abundances and previous analyses are not very significant, especially since our analysis is not based on newly observed spectra. Our work does not aim at showing that, with our consistently determined temperature scale, we can now better describe the properties of the $A(\mathrm{Li})$-plateau. On the contrary, our analysis has so far pointed out that although this is clearly a must, even with such a careful determination of the temperature scale, many uncertainties remain especially for large samples.

However, since the opposite findings by some earlier analyses (e.g. R96, R99, and BM97) have indeed been among the initial triggers of this work, we think it is useful to further comment on few points.
Table 7. Discrepant cases in the original works of R96, R99, and BM97. Columns identified by a $\Delta$ symbol represent the difference on that given quantity $\left(T_{\mathrm{eff}},[\mathrm{Fe} / \mathrm{H}], E W\right.$, and lithium abundance) between R96 and BM97 (when a target has a second entry, this refers to R99 BM97).

\begin{tabular}{rrcccc}
\hline \hline HIP & $\Delta T_{\text {eff }}$ & $\Delta[\mathrm{Fe} / \mathrm{H}]$ & $\Delta E W$ & $\Delta A(\mathrm{Li})$ & $\Delta A(\mathrm{Li})_{\text {end }}^{a}$ \\
& $\mathrm{~K}$ & $\mathrm{dex}$ & $\mathrm{m} \AA$ & $\mathrm{dex}$ & $\mathrm{dex}$ \\
\hline 3430 & +144 & -0.29 & 0.0 & +0.09 & -0.02 \\
11952 & -94 & -0.11 & 0.0 & -0.08 & -0.01 \\
12807 & -207 & +0.51 & 0.0 & -0.11 & -0.03 \\
& -287 & +0.58 & +5.9 & -0.11 & -0.05 \\
14594 & -200 & +0.05 & 0.0 & -0.18 & -0.04 \\
23344 & +230 & -0.90 & 0.0 & +0.15 & 0.01 \\
& +130 & -0.77 & -1.5 & +0.05 & 0.02 \\
42592 & -164 & +0.20 & 0.0 & -0.12 & -0.01 \\
& -186 & -0.02 & -2.9 & -0.18 & 0.01 \\
44605 & -240 & -0.34 & 0.0 & -0.23 & -0.05 \\
66673 & -178 & -0.15 & 0.0 & -0.10 & 0.03 \\
& -248 & +0.18 & -5.8 & -0.25 & 0.06 \\
68592 & -142 & -0.49 & 0.0 & -0.09 & 0.02 \\
& -192 & -0.72 & +3.2 & -0.01 & 0.07 \\
78640 & -179 & +0.17 & 0.0 & -0.17 & -0.05 \\
87693 & -361 & -0.25 & 0.0 & -0.25 & 0.01 \\
96115 & -160 & +0.18 & 0.0 & -0.11 & 0.00 \\
& -160 & +0.04 & +1.6 & -0.11 & -0.03 \\
114962 & -142 & +0.23 & 0.0 & -0.15 & -0.06 \\
\hline
\end{tabular}

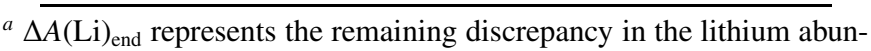
dance after having taken into account the differences in $T_{\text {eff }}(\mathrm{Col} .2)$, [Fe/H] (Col. 3), and $E W(\mathrm{Col} .4)$.

First of all, we note that most of the discrepancies originally present among some stars common to R96, R99, and BM97 (which some of the earlier claims for dispersion and/or slopes may have originated from) can be fully explained by differences in the stellar parameters adopted during the various analyses. Table 7 summarises these comparisons for some objects common to these works, with Cols. 2 to 5 reporting the differences in $T_{\text {eff }}$, metallicity, $E W$ (if any), and the $A(\mathrm{Li})$ abundance as reported by the original investigators (always given as "R96-BM97"). When, for a given target, a double entry is present, this second row of values corresponds to "R99-BM97". The last column of the table reports the remaining difference in the final $A(\mathrm{Li})$ value, after having taken into account the differences in $T_{\text {eff }},[\mathrm{Fe} / \mathrm{H}]$, and $E W$.

This is indeed very positive, but in general we find a little worrysome the revisions made by R99 to some of their measured equivalent widths, compared to their own previous analysis from 1996. Although both analyses are based on high quality, high $S / N$ spectra, $E W$ measurements of the Li I line for the same star differ by as much as $6 \mathrm{~m} \AA$ which for the $T_{\text {eff }}$ range covered by the R99 sample correspond to 0.06 dex in lithium abundance.

Also, the effective temperatures of the 10 stars common to the analyses of R99, BM97, and this work span respectively $220 \mathrm{~K}, 358 \mathrm{~K}, 520 \mathrm{~K}$. In other words, the data-sample that Ryan et al. carefully chose to span a very narrow range of effective temperatures (and that indeed did so on their $T_{\text {eff }}$ scale), it is 
found to cover a much larger interval when the temperature is derived following different prescriptions. And all three analyses used self-consistent methods!

Another example stressing the weakness of the temperature scale issue comes from the comparison of our results with the recent work by Meléndez \& Ramírez (2004). The latter have carried out a study similar to ours, in which 62 halo dwarfs (of which, in the end, 41 were used to discuss the mean Li abundance of the plateau) were analysed based on a newly derived and improved IRFM-based $T_{\text {eff }}$ scale (Ramírez \& Meléndez 2005a,b) and using $\mathrm{Li}$ equivalent width measurements available in the literature. A quick check between the effective temperatures reported in their Table 1 (Meléndez \& Ramírez 2004) and our values, for the 32 stars we have in common, shows both an offset (their temperature scale is hotter) and a larger temperature interval spanned $(737 \mathrm{~K}$ versus $583 \mathrm{~K}$, the latter from our analysis). The offset implies that their mean Li-plateau value will be higher than ours. The fact that their temperature scale is hotter than ours is not in contradiction with what is shown in the bottom panel of Fig. 11, where a much larger sample of stars is plotted.

Still related to the determination of an accurate temperature scale, and as already seen in Sect. 5, reddening plays an important role. For instance, R99 noted that their reddening estimates based on two different methods (Strömgren photometry and reddening maps) showed a clear discrepancy of about $0.02 \mathrm{mag}$, with the Strömgren-based $E(b-y)$ values being higher despite the expected relationship $E(B-V) \simeq$ $1.35 E(b-y)$. The solution chosen by these authors was to give higher weight to the maps-based reddening values based on the consideration that their targets were bright, hence a low intrinsic reddening might be expected. Therefore, they systematically lowered all the Strömgren-based $E(b-y)$ values by 0.02 mag before averaging the two methods. As we do not know the final answer either, we cannot say if this is a good solution or not. At least for those objects we have in common these stars fall well outside the inner $50-70 \mathrm{pc}$ of the solar neighborhood (they span distances up to $1 \mathrm{kpc}$ ), for which a low intrinsic reddening could be questionable.

Even more instructive is to evaluate the realistic situation in which one may refold on selecting reddening values from different sources, such as InfraRed dust maps and/or from the literature, because one method alone cannot be applied homogeneously to the entire sample under investigation. Keeping in mind that the exact details of such situation are very difficult to foresee, hence to reproduce, we tried to mimick such case by plotting a mix of lithium abundances: Fig. 12 shows $A(\mathrm{Li})_{\text {NLTE }}$ versus $T_{\text {eff }}$, where the lithium abundances were derived by selecting $T_{\text {eff }}(2)$ and $T_{\text {eff }}$ (S98) for the clean sample (half-half), and $T_{\text {eff }}$ (H97) and $T_{\text {eff }}$ (mix) for the $\beta$ and $u b v y$ samples respectively. This likely represents an extreme case, but it certainly gives an idea of what effect could be expected. Also, please note that the $A(\mathrm{Li})$ values corresponding to $T_{\text {eff }}$ (mix) were computed only for those stars for which this solution was applied (cf. Sects. 5.1 and 5.2).

In summary, despite the seeming convergence at least on the absence of dispersion, the finding of discordant results is not surprising if some (or all) of the above-mentioned points

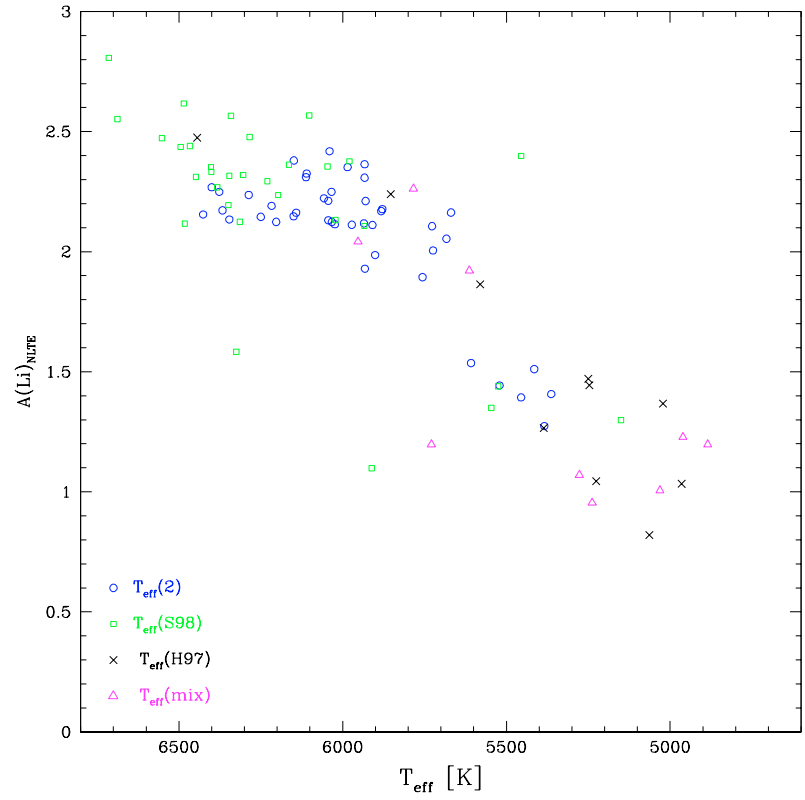

Fig. 12. Lithium abundances as determined by using the sets of temperatures $T_{\text {eff }}$ (2) and $T_{\text {eff }}$ (S98) for the clean sample (half-half, circle and square symbols respectively), $T_{\text {eff }}$ (H97) and $T_{\text {eff }}$ (mix) for the $\beta$ and $u b v y$ sample (crosses and triangles respectively), in the attempt of mimicking a common situation in which reddenings are taken from different sources.

are kept in mind. At the moment the only claim for a tilted

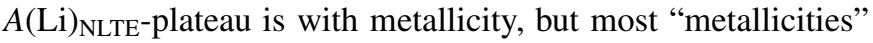
quoted in the literature are still derived from neutral iron lines, the formation of which is subject to NLTE conditions. Furthermore, R99 (as well as our work) have used metallicity values extracted from a careful inspection of the literature, which does not guarantee the homogeneity required for discussing the $A(\mathrm{Li})$ vs. $[\mathrm{Fe} / \mathrm{H}]$ trend. Since the whole discussion of a possible slope of the lithium plateau with metallicity is centred on a small dependence, we encourage future analyses of $\mathrm{Li}$ abundances to determine the metallicities spectroscopically, possibly from Fe II lines (insensitive to NLTE effects).

\section{Preliminary results on the mean lithium abundance and the dispersion}

\subsection{Last words of caution before further analysis}

A first visual comparison of the different $A(\mathrm{Li})_{\text {NLTE }}$ abundances we have derived for each star is presented in Fig. 13, where the first three (from the top) panels show how differently the plateau may appear when the lithium abundance has been derived from a different set of temperatures. The bottom pan is shown only for comparison purposes, and represents the $A(\mathrm{Li})_{\text {NLTE }}$ abundances as derived from photometry that has been dereddened using the BH H I maps. Figure 13 shows how claims of dispersion or lack thereof from the same data-sample are perfectly plausible (see also our discussion in Sect. 7).

In addition, since our complete sample is a nonhomogeneous sample (because of the compromises made on the derivation of the effective temperature for some of the stars), any discussion of the width and slope of the 


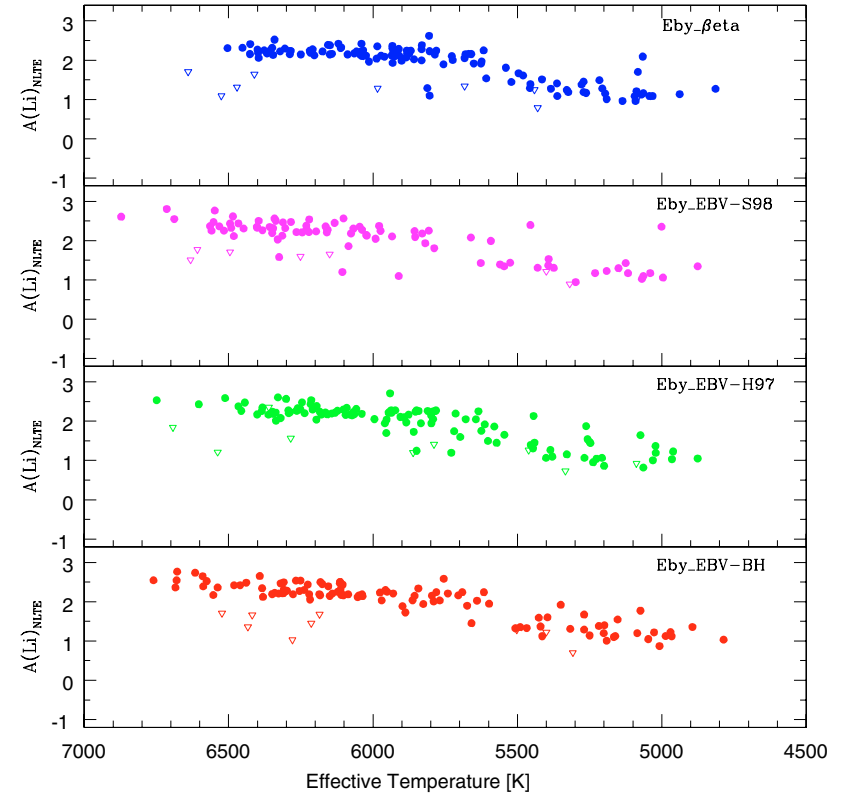

Fig. 13. Lithium abundances computed with four different sets of temperatures as derived by using different reddening estimates (as reported in the upper right corner of each pan). Upside-down open triangles represent abundance upper limits.

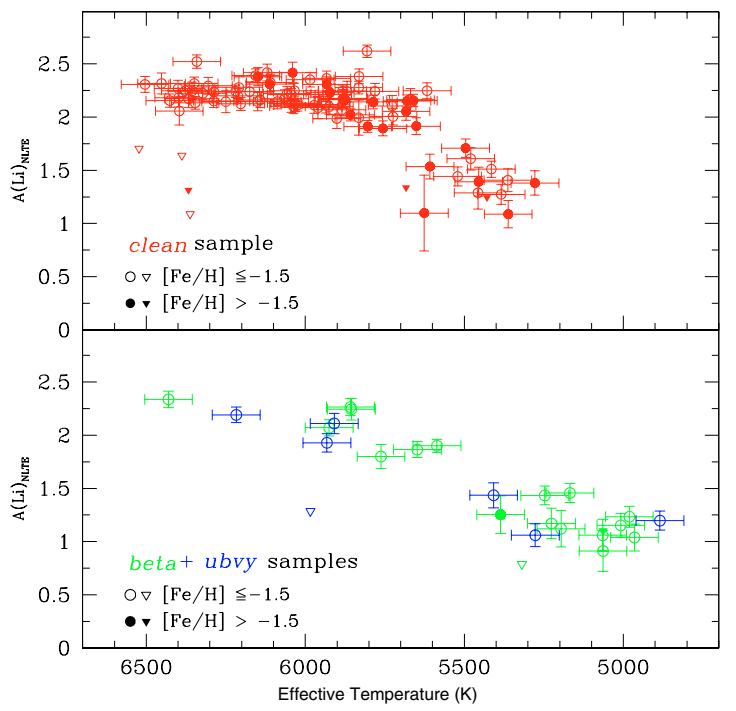

Fig. 14. Final lithium abundances with associated $1 \sigma$-errorbars as a function of effective temperature (using $T_{\text {eff }}(2)$ ) are shown for our clean sample (top) and beta + ubvy samples (bottom).

$A(\mathrm{Li})$-plateau requires the separation of the complete sample into the clean, $\beta$, and $u b v y$ sub-samples. In Figs. 14 and 15, we plot the $A(\mathrm{Li})_{\mathrm{NLTE}}$ values vs. our $T_{\text {eff }}$ scale (i.e. $\left.T_{\text {eff }}(2)\right)$ and $[\mathrm{Fe} / \mathrm{H}]$ respectively, with the clean sample always plotted in the top panel and the $\beta$ and $u b v y$ samples in the lower panel. Moreover, open symbols refer to objects with $[\mathrm{Fe} / \mathrm{H}] \leq-1.5$ in Fig. 14 and $T_{\text {eff }} \geq 5700 \mathrm{~K}$ in Fig. 15, and filled symbols represent respectively stars with $[\mathrm{Fe} / \mathrm{H}]>-1.5$ and $T_{\text {eff }}<5700 \mathrm{~K}$. Upside-down triangles always identify abundance upper limits.

In the rest of the paper, we give our results regarding the characteristics of the plateau for the clean sample on one hand, and for the complete (i.e., clean $+\beta+u b v y$ ) sample on the other

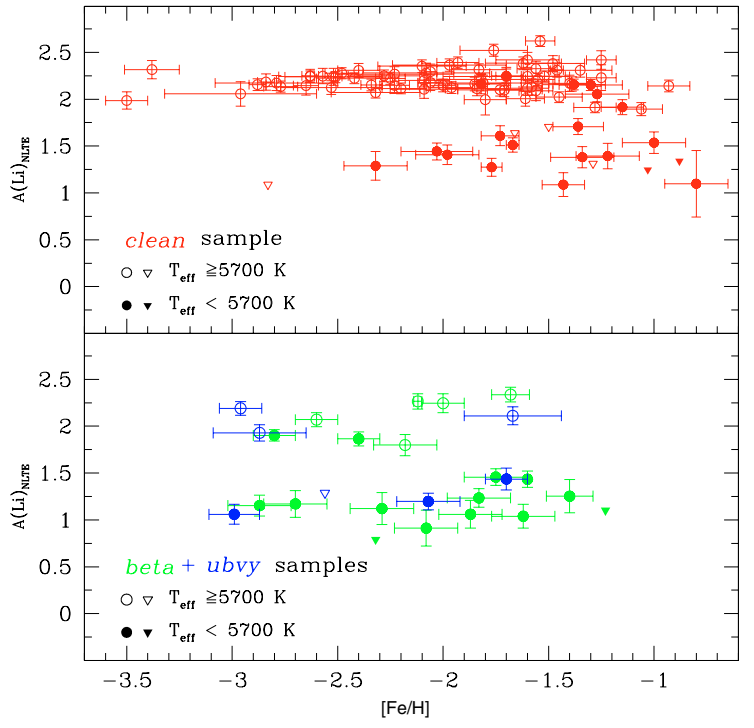

Fig. 15. Final lithium abundances with associated $1 \sigma$-errorbars as a function of metallicity (using $T_{\text {eff }}(2)$ ) are shown for our clean sample (top) and for our beta + ubvy samples (bottom).

hand. Additionally, we will consider as "plateau stars" those with $T_{\text {eff }} \geq 5700 \mathrm{~K}$ and $[\mathrm{Fe} / \mathrm{H}] \leq-1.5$. The metallicity limit is taken in order to avoid any contamination by lithium production from the various possible stellar sources (e.g., Travaglio et al. 2001). The cutoff in $T_{\text {eff }}$ is chosen for comparison reasons with previous analyses in the literature. However for the purposes of constraining stellar evolution models we will also discuss the cases where the lower limit in $T_{\text {eff }}$ is increased to $6000 \mathrm{~K}$ in order to avoid proto-stellar lithium destruction (in the case of the dwarfs, see Sect. 10) or dilution at the very beginning of the first dredge-up phase (in the case of the post-turnoff stars, see Sect. 11). Our results will be given considering the stars with lithium abundance determinations only, the case of stars with upper limits being considered separately in Sect. $13^{7}$.

\subsection{The mean lithium plateau abundance}

Considering the stars with $[\mathrm{Fe} / \mathrm{H}] \leq-1.5$ and in the case of the most relaxed $T_{\text {eff }}$ lower limit (5700 K, cf. Figs. 14 and 15) we obtain

$A(\mathrm{Li})_{\mathrm{NLTE}}=2.2138 \pm 0.0929$

and

$A(\mathrm{Li})_{\mathrm{NLTE}}=2.2080 \pm 0.0947$

for the clean and complete samples respectively, with rms values of 0.0587 and 0.0530 . This is compatible with a normal distribution (i.e., as would be expected from the observational errors).

For the stars with $T_{\text {eff }} \geq 6000 \mathrm{~K}$, we find a mean lithium abundance of

$A(\mathrm{Li})_{\mathrm{NLTE}}=2.2243 \pm 0.0748$

${ }^{7}$ For the metallicity and effective temperature range of the plateau, only three stars have Li upper limits, namely HIP 72561, 81276 and 100682. 
for the clean sample, and of

$A(\mathrm{Li})_{\mathrm{NLTE}}=2.2368 \pm 0.0840$

for the complete sample. In both cases the dispersion values are slightly higher than the rms of the estimated observational error (0.0587), but compatible with no dispersion on the plateau.

Similar conclusions on dispersion can be drawn if no lower limit on $[\mathrm{Fe} / \mathrm{H}]$ (in our specific case this was set to -2.1) is assumed in the $T_{\text {eff }}-(b-y)_{0}$ calibration (see Sect. 5 for more details). The higher effective temperatures thus derived and shown in Fig. 3 would have the only effect of increasing our mean $A(\mathrm{Li})$ plateau values by $0.025-0.035 \mathrm{dex}$.

We would like to note that the 6 stars that could be moved to the $u b v y$ sample (if we were to consider a 3 digit precision on $\beta$, cf. end of Sect. 5.2) are not relevant in the final discussion of the Li plateau spread as they are cool stars that have their lithium already depleted ( 5 of them) or a normal plateau lithium abundance ( 1 star with $A(\mathrm{Li})_{\mathrm{NLTE}}=2.22$ ).

The absence of intrinsic dispersion that we get is in agreement with the results of Molaro et al. (1995), Spite et al. (1996), Bonifacio \& Molaro (1997), Ryan et al. (1999) and Meléndez \& Ramírez (2004).

Furthermore, because all these works (ours included) find quite consistent $A(\mathrm{Li})$ plateau values (note that the apparently higher plateau value found by Meléndez \& Ramírez (2004, $\left.A(\mathrm{Li})_{\mathrm{NLTE}}=2.37\right)$ should be corrected downwards by $0.08 \mathrm{dex}$ because of the different Kurucz model atmospheres employed in their and our study, making it then in closer agreement with our findings), it seems that in general the relatively low lithium abundance (when compared to the $\mathrm{CMB}+\mathrm{SBBN}$ result) seen in metal poor halo stars is a very robust result. Assuming the correctness of the CMB constraint on the value of the baryonto-photon ratio we are then left with the conclusion that the $\mathrm{Li}$ abundance seen at the surface of halo stars is not the pristine one, but that these stars have undergone surface lithium depletion at some point during their evolution.

Let us try now to look for some constraints on the depletion mechanism(s).

\section{Evolutionary status of the stars}

Using the data we have gathered and homogenized in the first part of this paper, we will now look at the Li plateau by adding one extra dimension to the problem, namely by considering the evolutionary status of each star of our complete sample. Indeed not all our objects are dwarf stars. The contamination from evolved stars has thus to be evaluated in order to precisely determine the lithium abundance along the plateau and to look for the trends and for the depletion factor.

\subsection{Input quantities}

We use the HIPPARCOS (ESA 1997) trigonometric parallax measurements to locate precisely our objects in the HR diagram. Among the 118 stars of our complete sample, 3 objects (HIP 484, 48444, and 55852) have spurious Hipparcos parallaxes, and are thus rejected from further analysis.
Table 8. Quantities extracted from the HIPPARCOS catalogue for our sample stars $((B-V)$ and $E(B-V)$ are given in Table 4$)$. The whole table is available on-line.

\begin{tabular}{cccccc}
\hline \hline HIP & $\begin{array}{c}V \\
\text { mag }\end{array}$ & $\begin{array}{c}\text { Plx } \\
(\mathrm{mas})\end{array}$ & $\begin{array}{c}\text { e_Plx } \\
(\mathrm{mas})\end{array}$ & $\begin{array}{c}d \\
(\mathrm{pc})\end{array}$ & VI \\
\hline 911 & 11.80 & 6.13 & 5.67 & 163 & 0.64 \\
3026 & 9.25 & 9.57 & 1.38 & 104 & 0.54 \\
3446 & 12.10 & 15.15 & 3.24 & 66 & 0.58 \\
3564 & 10.60 & 2.07 & 2.16 & 483 & 0.67 \\
8572 & 10.34 & 3.22 & 1.75 & 310 & 0.50 \\
$\cdots$ & $\ldots$ & $\cdots$ & $\cdots$ & $\cdots$ & $\cdots$ \\
\hline
\end{tabular}

Intrinsic absolute magnitudes $M_{V}$ are derived from the $m_{V}$ and the parallaxes given in the Hipparcos catalogue. We determine the bolometric corrections $\mathrm{BC}$ by using the relations between $\mathrm{BC}$ and $V-I$ (these quantities being also taken from the Hipparcos catalogue) given by Lejeune et al. (1998) and which are metallicity-dependent. We use the values of $[\mathrm{Fe} / \mathrm{H}]$ derived in our final analysis (Table 6, Col. 2). We first iterate using the $\log g$ values available in the literature, and finally attribute to our stars the $\log g$ value derived from their position in the HR diagram (Table 9, Col. 10). Finally, we compute the stellar luminosity and the associated error from the one sigma error on the parallax. All the relevant quantities are given in Tables 8 and $9^{8}$.

\subsection{Determination of the stellar evolutionary status}

The resulting HR diagrams are shown in Fig. 16 for our complete sample split in four metallicity bins. The evolutionary status of each star has been determined on the basis of these HR diagrams, and is given in Table 9. Each star has been assigned to one of the following sub-classes: 5 identifies the dwarfs (i.e., main-sequence stars), 4.5 stands for the turnoff stars, 4 for the subgiants (i.e., stars crossing the Hertzsprung gap), 3.5 for the stars at the base of the RGB, and 3 for the stars on the RGB. "Post-main sequence stars" can be easily located on the HRD (see Fig. 16) as those stars with $\log \left(L / L_{\odot}\right)$ higher than $\sim 0.4$. In this luminosity range, we identify as turnoff and subgiant stars those with $T_{\text {eff }} \geq 5600 \mathrm{~K}$, whereas cooler stars are classified as "base RGB" or RGB stars depending on their luminosity. This limit in effective temperature is chosen because it corresponds to the approximate value where lithium dilution is expected to occur at the very beginning of the first dredge-up (i.e., Deliyannis et al. 1990; Charbonnel 1995). The distribution in these sub-classes is given in Table 10. For most of our objects, the classification was performed unambiguously. Some stars (21 in total) were first classified as "unsure", the uncertainty being due to the large errorbar on the derived luminosity inherited from the error on the Hipparcos parallax (see Fig. 16). However, by cross-checking the luminosity and the corresponding gravity obtained from Hipparcos data with the $M_{V}$ and gravity values quoted in the literature, we were able to attribute a relatively certain evolutionary status (given

\footnotetext{
8 Available in their entirety on-line.
} 
Table 9. Characteristics and evolutionary status of the sample stars. The whole table is available on-line.

\begin{tabular}{cccccccccc}
\hline \hline HIP & $M_{V}$ & $\begin{array}{c}M_{V} \\
\text { dered }\end{array}$ & BC & $M$ bol & $\log \left(\frac{L}{L_{\odot}}\right)$ & $\begin{array}{c}\log \left(\frac{L}{L_{\odot}}\right) \\
\text { dered }\end{array}$ & e_Log $\left(\frac{L}{L_{\odot}}\right)$ & $\log g$ & Status $^{s}$ \\
\hline 911 & 5.74 & 5.74 & -0.176 & 5.56 & -0.32 & -0.32 & 0.80 & 4.50 & 5 \\
3026 & 4.15 & 4.15 & -0.169 & 3.99 & 0.31 & 0.31 & 0.13 & 3.85 & 5 \\
3446 & 8.00 & 8.00 & -0.085 & 7.92 & -1.27 & -1.27 & 0.19 & 4.50 & 5 \\
3564 & 2.18 & 2.06 & -0.221 & 1.96 & 1.12 & 1.17 & 0.91 & 3.50 & 4 \\
8572 & 2.88 & 2.79 & -0.112 & 2.77 & 0.79 & 0.83 & 0.47 & 3.85 & 4 \\
$\ldots$ & $\ldots$ & $\ldots$ & $\ldots$ & $\ldots$ & $\ldots$ & $\cdots$ & $\cdots$ & $\cdots$ & $\cdots$ \\
\hline
\end{tabular}

(s) Status : 5:dwarf - 4.5:turnoff - 4: subgiant - 3.5:base RGB - 3:RGB.

(see the text for more details on the adopted definitions of these statuses).

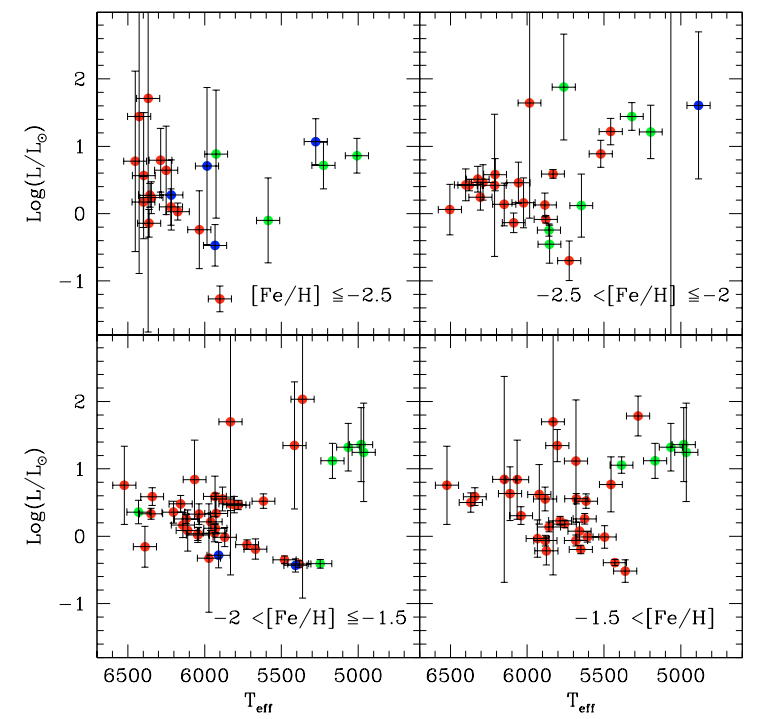

Fig. 16. HR diagram for our complete sample stars for separate metallicity bins (using $T_{\text {eff }}(2)$ ).

in the last row of Table 10) to all these objects, thus keeping them in our statistical analysis. As a result, the clean sample (respectively the complete sample) contains 49 (59) dwarfs, 5 (5) turnoff stars, 29 (31) subgiants, 5 (18) base RGB stars and 2 (5) RGB stars.

In Fig. 17 we plot $A(\mathrm{Li})_{\text {NLTE }}$ vs. $T_{\text {eff }}$ for four metallicity bins and indicate the evolutionary status of the stars by different symbols. It is interesting to note that for $[\mathrm{Fe} / \mathrm{H}] \leq-2$, all the coolest stars (i.e., with $T_{\text {eff }} \leq 5500 \mathrm{~K}$ ) are actually evolved stars (see also Fig. 18). This fact must be taken into account when comparing stellar evolution models and observations. In the $[\mathrm{Fe} / \mathrm{H}]$ range between -2 and -1.5 , the decrease of lithium relative to the plateau dwarf stars appears at $\sim 5600-5700 \mathrm{~K}$.

\subsection{Comparison with previous work}

In their careful analysis of the classical mechanisms ${ }^{9}$ that could alter the surface Li abundance of halo stars at different phases

\footnotetext{
9 Nuclear burning at the basis of the convective envelope on the pre- and early-main sequence, diffusion by gravitational settling on the
}

Table 10. Distribution of the evolutionary status in our different subclasses. The last line indicates the status that we could attribute to the stars which were first labelled as "unsure" (see the text for more details).

\begin{tabular}{ccc}
\hline \hline & \multicolumn{2}{c}{ Sample } \\
\hline total & 90 & Polluted (beta + ubvy $)$ \\
dwarfs & 44 & $27(20+7)$ \\
turnoff & 3 & $10(6+4)$ \\
subgiant & 18 & 0 \\
base RGB & 5 & $2(1+1)$ \\
RGB & 1 & $13(11+2)$ \\
unsure & 19 & 0 \\
& {$[5 \mathrm{dw}, 2$ to, $11 \mathrm{sg}, 1 \mathrm{rgb}]$} & $2(2+0)$ \\
\hline
\end{tabular}

of their evolution, Deliyannis et al. (1990, hereafter D90) focused on the separation of the halo population into pre- and post-turnoff groups as an essential prerequisite to understand the Li observations. At that time they considered a limited sample of halo stars. They used the trigonometric parallaxes and $V$-colors from the Yale Parallax Catalogue (van Altena et al. 1989) when available to determine $M_{V}$; otherwise $M_{V}$ was taken from the original observational papers. We have 36 objects in common with D90's sample. For 19 of them the same evolutionary status has been attributed in both D90 and our study. However 13 of the stars which were identified as "preturnoff" objects by D90 appear to be more evolved stars on the basis of their more precise and reliable Hipparcos parallaxes. Conversely 4 out of the 7 stars which were claimed to be "postturnoff" objects in D90's study are dwarf stars.

Ryan \& Deliyannis (1998) took advantage of the Hipparcos parallaxes (when available) in order to separate their sample of halo stars cooler than the plateau into dwarf and subgiant classes. The 14 stars that we have in common with their sample have been attributed the same evolutionary status in both analyses (theirs and ours).

Note that our definition of a subgiant is different and more strict, as far as the effective temperature range is concerned,

main sequence and at the turnoff, convective dredge-up on the early post-main sequence, and dilution on the post-main sequence. 


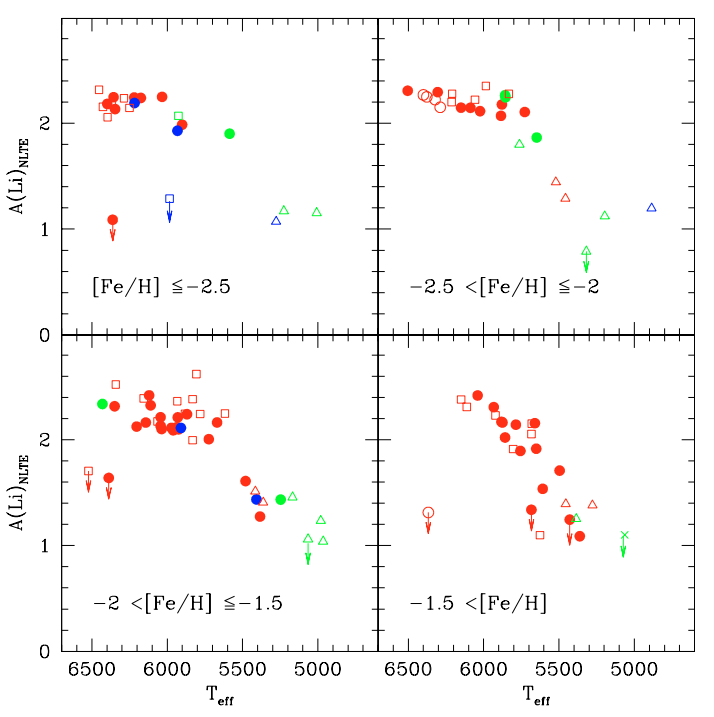

Fig. 17. $A(\mathrm{Li})_{\text {NLTE }}$ versus $T_{\text {eff }}$ (2) for our complete sample stars, for separate metallicity bins. The different symbols indicate the evolutionary status of the stars. Filled circles: dwarfs. Open circles: turnoff stars. Open squares: subgiants. Open triangles: stars at the base of the RGB. Crosses: RGB stars. The arrows indicate the lithium upper limits.

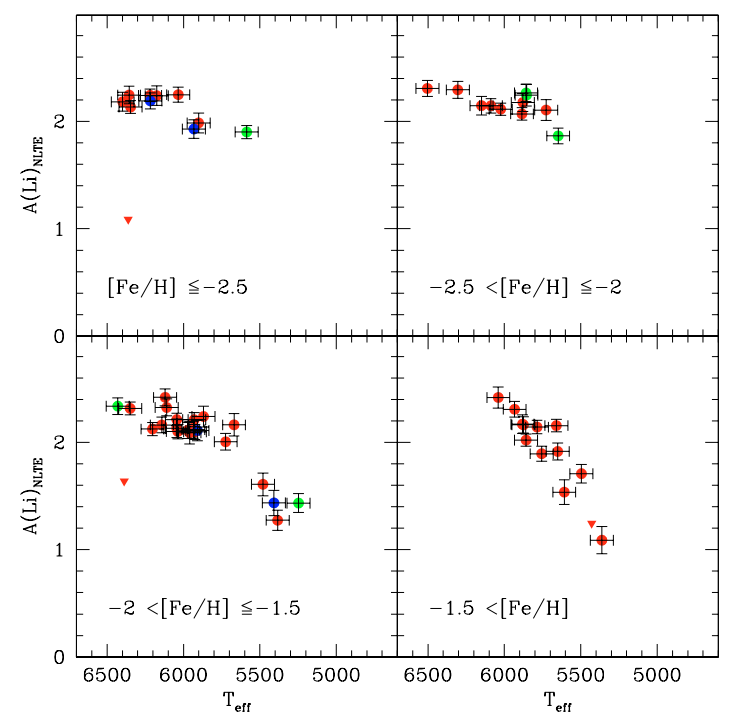

Fig. 18. $A(\mathrm{Li})_{\mathrm{NLTE}}$ versus $T_{\text {eff }}$ (2) for the dwarfs of our complete sample. Upside-down triangles represent lithium upper limits.

than the one adopted by PSB93, who studied the lithium abundances for 79 so-called halo subgiants. PSB93 constructed their sample on the basis of $u b v y$ photometry (more precisely from their location in the $c_{1}$ vs. ( $\left.b-y\right)$ plane) from several catalogs of metal-poor stars. Hipparcos parallaxes are now available for almost all their objects, allowing a more precise determination of their location in the HRD. In the present study we did not consider the most evolved stars of PSB93's sample, i.e., those with $T_{\text {eff }}$ below $4800 \mathrm{~K}$. These objects indeed experience some extra-mixing beyond the first dredge-up and lose then all the information about their initial lithium content (see Charbonnel 1995; Weiss \& Charbonnel 2004). They are thus of no help in discussing the initial lithium content.
Table 11. Number of dwarfs in each subsample.

\begin{tabular}{ccccc}
\hline \hline$[\mathrm{Fe} / \mathrm{H}]$ & Clean & $\begin{array}{c}\text { Polluted } \\
\text { (beta }+u b v y) \\
>5700\end{array}$ & $\begin{array}{c}\text { Clean } \\
>5700\end{array}$ & $\begin{array}{c}\text { Polluted } \\
\text { (beta }+u b v y) \\
>6000\end{array}$ \\
\hline$\leq-2.5$ & 8 & $0+2$ & 7 & $0+1$ \\
-2.5 to -2.0 & 9 & $2+0$ & 6 & $0+0$ \\
-2.0 to -1.5 & 16 & $1+1$ & 9 & $1+0$ \\
\hline
\end{tabular}

Table 12. Average value of $A(\mathrm{Li})_{\mathrm{NLTE}}$, average and standard deviation, and rms for the dwarf stars : mean $n_{ \pm s t a n d}^{ \pm}(\mathrm{rms})$. We separate the clean and the complete sample and give the results for 2 limits in effective temperature.

\begin{tabular}{ccc}
\hline \hline$[\mathrm{Fe} / \mathrm{H}]$ & \multicolumn{2}{c}{$T_{\text {eff }} \geq 5700 \mathrm{~K}$} \\
& clean & complete \\
\hline$\leq-2.5$ & $2.183_{ \pm 0.097}^{ \pm 0.070}(0.042)$ & $2.156_{ \pm 0.119}^{ \pm 0.093}(0.052)$ \\
-2.5 to -2.0 & $2.170_{ \pm 0.087}^{ \pm 0.067}(0.047)$ & $2.187_{ \pm 0.075}^{ \pm 0.072}(0.057)$ \\
-2.0 to -1.5 & $2.178_{ \pm 0.109}^{ \pm 0.088}(0.042)$ & $2.183_{ \pm 0.092}^{ \pm 0.092}(0.067)$ \\
$\leq-1.5$ & $2.177_{ \pm 0.098}^{ \pm 0.071}(0.047)$ & $2.177_{ \pm 0.104}^{ \pm 0.084}(0.052)$ \\
\hline \multicolumn{3}{c}{$T_{\text {eff }} \geq 6000 \mathrm{~K}$} \\
\hline \multicolumn{3}{c}{ clean } \\
\hline$\leq-2.5$ & $2.216_{ \pm 0.047}^{ \pm 0.038}(0.042)$ & $2.212_{ \pm 0.044}^{ \pm 0.037}(0.052)$ \\
-2.5 to -2.0 & $2.202_{ \pm 0.091}^{ \pm 0.079}(0.047)$ & $2.202_{ \pm 0.091}^{ \pm 0.079}(0.047)$ \\
-2.0 to -1.5 & $2.225_{ \pm 0.108}^{ \pm 0.088}(0.042)$ & $2.236_{ \pm 0.115}^{ \pm 0.100}(0.067)$ \\
$\leq-1.5$ & $2.215_{ \pm 0.088}^{ \pm 0.074}(0.047)$ & $2.200_{ \pm 0.088}^{ \pm 0.074}(0.052)$ \\
\hline
\end{tabular}

\section{The lithium abundance along the plateau for the dwarf stars}

We now concentrate our analysis on the dwarf stars. Their lithium abundance is plotted in Fig. 18.

\subsection{The analytical method}

For the reasons given in Sect. 8.1, we identify as "plateau stars" those with $[\mathrm{Fe} / \mathrm{H}] \leq-1.5$ and with $T_{\text {eff }}$ higher than 5700 or $6000 \mathrm{~K}$. The corresponding numbers of stars in each bin is given in Table 11. For the time being, we keep in our analysis the possible binary stars (but see Sect. 12), but we eliminate the stars with lithium upper limits, that will be discussed separetely in Sect. 13. We note that in the metallicity and effective temperature ranges we have chosen to define the plateau only two dwarf stars of the clean sample have Li upper limits, namely HIP 72561 and 100682. With $A(\mathrm{Li})_{\mathrm{NLTE}} \leq 1.639$ and $\leq 1.088$ respectively (with $[\mathrm{Fe} / \mathrm{H}]=-1.66$ and $-2.83, T_{\text {eff }}=6388$ and $6362 \mathrm{~K}$ ), both stars fall well below the plateau (see Fig. 18).

In order to investigate the existence of trends in the $\left[A(\mathrm{Li})_{\mathrm{NLTE}}, T_{\text {eff }}\right]$ and $\left[A(\mathrm{Li})_{\mathrm{NLTE}},[\mathrm{Fe} / \mathrm{H}]\right]$ planes we performed univariate fits by means of four estimators: (1) The leastsquares fit with errors in the independant variable only (routine FIT of Press et al. 1982); (2) The least-squares fit with errors in both variables (routine FITEXY of Press et al. 1982); (3) The BCES (bivariate correlated errors and intrinsic scatter) of Akritas \& Bershady (1996); (4) BCES simulations bootstrap based on 10000 samples. All univariate fits in the $A(\mathrm{Li})_{\mathrm{NLTE}}$ 
versus $T_{\text {eff }}$ plane were computed considering an error on $T_{\text {eff }}$ of both 75 and $100 \mathrm{~K}$.

\subsection{Mean value and dispersion}

The average value of the lithium abundance is given in Table 12, together with the average, standard deviation and root mean square of the estimated observational errors for several subsets of data (in terms of metallicity and effective temperature intervals, as well as in terms of clean vs. complete samples) of the dwarf sample.

If we consider the entire metallicity range $[\mathrm{Fe} / \mathrm{H}] \leq-1.5$, the straight average value of the lithium abundance for the plateau dwarf stars with $T_{\text {eff }} \geq 5700 \mathrm{~K}$ is

$A(\mathrm{Li})_{\mathrm{NLTE}}=2.1768 \pm 0.0711$

and

$A(\mathrm{Li})_{\mathrm{NLTE}}=2.1773 \pm 0.0840$

for the clean and complete samples respectively. The average dispersion is small, but slightly higher than the respective rms of 0.0474 and 0.0516 .

When we restrict our analysis to the dwarf stars with $T_{\text {eff }} \geq 6000 \mathrm{~K}$, the mean value increases to

$A(\mathrm{Li})_{\mathrm{NLTE}}=2.2154 \pm 0.0737$

and

$A(\mathrm{Li})_{\mathrm{NLTE}}=2.2200 \pm 0.0740$

for the clean and the complete samples respectively. Again the average dispersion is small but slightly higher than the rms (0.0474 and 0.0516 respectively).

We thus find no evidence of an intrinsic dispersion in the Li abundances along the plateau. As expected, this result which was already presented in Sect. 8.2, is now reinforced having eliminated the "pollution" by evolved stars and focussed on the dwarf stars only.

As we already mentioned in Sect. 8.2, the assumption of a lower limit on $[\mathrm{Fe} / \mathrm{H}]$ in our $T_{\text {eff }}-(b-y)_{0}$ calibration does not affect our conclusions on dispersion. The only effect when one does not consider this limit is to increase the mean $A(\mathrm{Li})$ value of the dwarf sample by less than 0.02 dex for the entire metallicity range. If we consider only our most metal-poor subsamples the increase in the mean $A(\mathrm{Li})$ value is of course slightly higher (from 0.02 up 0.08 dex depending on the lowest limit on $T_{\text {eff }}$ used for the plateau definition).

The mean lithium values for the dwarf stars are slightly lower, although fully compatible within the quoted errors, than the mean values given in Sect. 8.2 for the entire sample of stars (i.e., that in which we did not discriminate the stars according to their evolutionary status). This point will be discussed at length in Sect. 11.

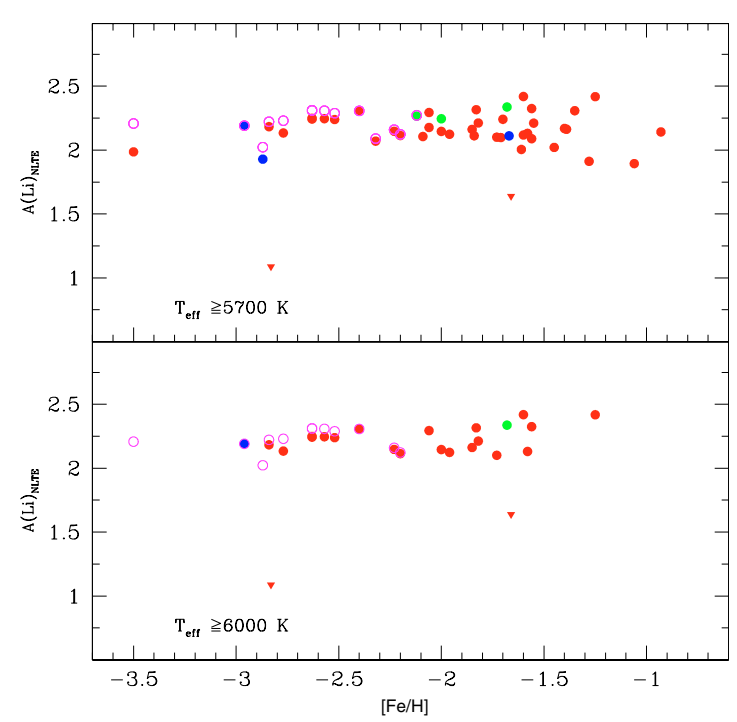

Fig. 19. $A(\mathrm{Li})_{\mathrm{NLTE}}$ versus $[\mathrm{Fe} / \mathrm{H}]$ only for the dwarfs with $T_{\text {eff }} \geq$ $5700 \mathrm{~K}$ (upper figure) and with $T_{\text {eff }} \geq 6000 \mathrm{~K}$ (lower figure) of our entire sample. Upside-down triangles represent abundance upper limits. The open circles correspond to the case where no lower limit on $[\mathrm{Fe} / \mathrm{H}]$ is assumed in the colour- $T_{\text {eff }}$ calibration, while the others correspond to the case where this limit is set to -2.1 .

\subsection{The $A(L i)_{\text {NLTE }}$ versus $T_{\text {eff }}$ correlation}

When we consider the dwarfs with $T_{\text {eff }} \geq 5700 \mathrm{~K}$ and $[\mathrm{Fe} / \mathrm{H}] \leq-1.5$, we find a small slope in the $A(\mathrm{Li})_{\mathrm{NLTE}}-T_{\text {eff }}$ plane of

\section{$0.019 / 100 \mathrm{~K}$ and $0.028 / 100 \mathrm{~K}$}

for the clean and complete samples respectively. These numbers are obtained when we use our standard error on $T_{\text {eff }}$ of $75 \mathrm{~K}$. In the more conservative case of an error on $T_{\text {eff }}$ of $100 \mathrm{~K}$, we get respectively $0.026 / 100 \mathrm{~K}$ and $0.033 / 100 \mathrm{~K}$.

This is very similar to the slope found by BM97 of $0.02 / 100 \mathrm{~K}$, and slightly lower than those found by Thorburn (1994) of $0.034 / 100 \mathrm{~K}$ and by R96 of $0.0408 / 100 \mathrm{~K}$. On the contrary, Meléndez \& Ramírez (2004) do not find any dependence of $A(\mathrm{Li})$ on either $T_{\text {eff }}$ or $[\mathrm{Fe} / \mathrm{H}]$.

In order to focus on the physical processes that may affect the surface lithium abundance only when the stars are on the main sequence (in other words, to avoid any pre-main sequence depletion; see e.g. D90, Richard et al. 2004) we made the same computations for the stars with $T_{\text {eff }} \geq 6000 \mathrm{~K}$. In this case and for the whole $[\mathrm{Fe} / \mathrm{H}] \leq-1.5$ range, we get a slightly smaller slope, i.e.

\section{$0.015 / 100 \mathrm{~K}$}

for the clean sample and a very similar slope, i.e.

\section{$0.029 / 100 \mathrm{~K}$}

for the complete sample, assuming an error on $T_{\text {eff }}$ of $75 \mathrm{~K}$. When we consider an error on $T_{\text {eff }}$ of $100 \mathrm{~K}$, we get respectively $0.027 / 100 \mathrm{~K}$ and $0.041 / 100 \mathrm{~K}$.

We note that if we split the $[\mathrm{Fe} / \mathrm{H}]$ interval as done for example in Fig. 18, we find a negative slope of $\sim-0.025$ for 
the stars with $[\mathrm{Fe} / \mathrm{H}] \leq-2.5$, both for the clean and complete samples for the stars with $T_{\text {eff }} \geq 6000 \mathrm{~K}$. Small but positive slopes are detected when we look at the $-2.5<[\mathrm{Fe} / \mathrm{H}] \leq-2.0$ and $-2.0<[\mathrm{Fe} / \mathrm{H}] \leq-1.5$ intervals.

\subsection{The $A(L i)_{\mathrm{NLTE}}$ versus $[\mathrm{Fe} / \mathrm{H}]$ correlation}

In Fig. 19 we plot $A(\mathrm{Li})_{\mathrm{NLTE}}$ vs. $[\mathrm{Fe} / \mathrm{H}]$ for the plateau dwarfs of our entire sample but we look for trends up to $[\mathrm{Fe} / \mathrm{H}]=-1.5$ only. The highest slope $(\sim 0.05$ up to $[\mathrm{Fe} / \mathrm{H}]=-1.5)$ is obtained for the most extended sample, i.e., for the dwarf stars of the complete sample with $T_{\text {eff }} \geq 5700 \mathrm{~K}$, whereas when we consider only the dwarf stars of the clean sample (still with $T_{\text {eff }} \geq 5700 \mathrm{~K}$ ) the correlation becomes slightly flatter (the slope is $\sim 0.02$ ). When we focus on the dwarf stars with $T_{\text {eff }} \geq 6000 \mathrm{~K}$, the dependence of $A(\mathrm{Li})$ with metallicity remains small (with a slope of $\sim 0.03$ and 0.02 dex up to $[\mathrm{Fe} / \mathrm{H}]=-1.5$ for the complete and clean samples respectively). In Fig. 19 we also show the values when no lower limit on $[\mathrm{Fe} / \mathrm{H}]$ is assumed in the $T_{\text {eff }}-(b-y)_{0}$ calibration. A slightly lower slope would have been derived under this assumption.

Our results are intermediate between those of BM97 $(-0.05$ to 0.00 , i.e., no slope) and those of R99 (+0.118) and Thorburn $(1994 ;+0.13)$. We note that the preliminary results of Asplund et al. (2005) indicate a dependence of $A(\mathrm{Li})$ on metallicity (the latter computed both as the abundance of iron and oxygen), characterised by a slope of 0.10 .

We remind the reader that lithium synthesis by galactic cosmic rays (GCR) leads indeed to an increase of its abundance with metallicity, but this contribution safely can be considered small up to $[\mathrm{Fe} / \mathrm{H}]=-1.5$ (Molaro et al. 1997) in agreement with our finding. This can be estimated using the observed correlation between ${ }^{9} \mathrm{Be}$ and metallicity together with the $\mathrm{Li} / \mathrm{Be}$ ratio predicted by GCR theory (Walker et al. 1985).

As discussed already in Sect. 7.2, the trends with metallicity here derived must be taken with caution, as we have gathered our metallicities from the literature. This does not guarantee the homogeneity required for an accurate study of the metallicity dependence. Additionally such an analysis would require homogeneous spectroscopic determinations from Fe II lines insensitive to NLTE effects rather than from neutral iron lines as it is the case for most of the currently available $\mathrm{Li}$ analyses (hence also for our values). For these reasons we will not continue the discussion nor derive any conclusions on the lithium-metallicity relation.

\subsection{The dwarf stars cooler than the plateau}

As already mentioned in Sect. 9.2, the current sample does not contain cool (i.e., with $T_{\text {eff }} \leq 5500 \mathrm{~K}$ ) dwarf stars at lowmetallicity (i.e., $[\mathrm{Fe} / \mathrm{H}] \leq-2$ ). This is the first surprise of our check on the evolutionary status of our sample stars. It thus appears that for the cooler and more metal-deficient dwarfs we have no direct clue to the lithium behavior. We expect these objects to exhibit the same pattern as that seen in cool open cluster and Pop I field stars as well as in our sample stars at higher metallicity (bottom panels of Fig. 18), although this remains to

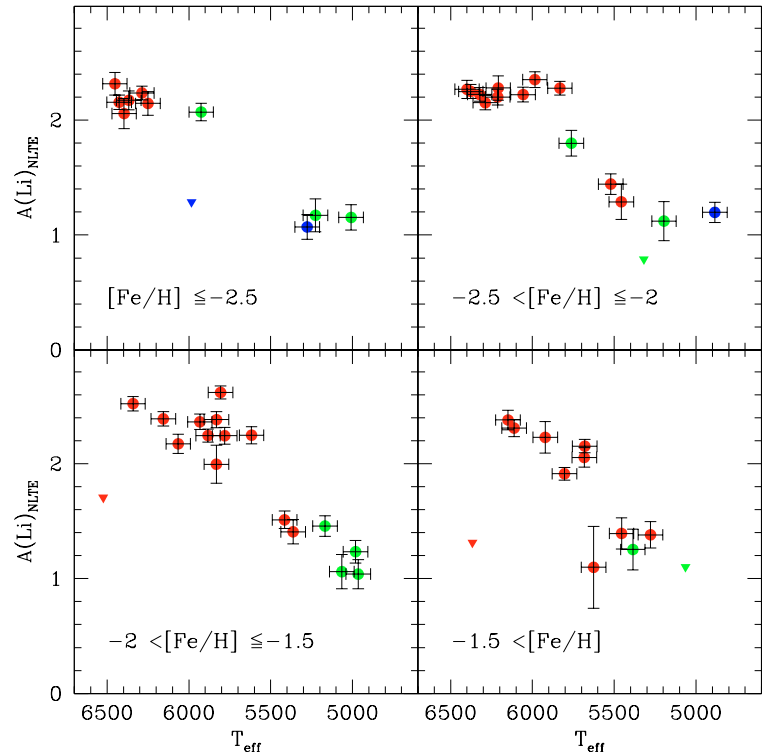

Fig. 20. $A(\mathrm{Li})_{\mathrm{NLTE}}$ versus $T_{\text {eff }}$ (2) for the turnoff and more evolved stars of our entire sample for four metallicity bins. Triangles are for lithium upper limits. Effective temperature is here an indicator of the evolutionary status of the stars.

Table 13. Straight average value of $A(\mathrm{Li})_{\mathrm{NLTE}}$, average and standard deviation for the turnoff and subgiant stars: mean $_{ \pm \text {stand }}^{ \pm \text {aver }}(\mathrm{rms})$. We separate the clean and the complete samples and present the results for 2 limits in effective temperature.

\begin{tabular}{ccc}
\hline \hline$[\mathrm{Fe} / \mathrm{H}]$ interval & \multicolumn{2}{c}{$T_{\text {eff }} \geq 5700 \mathrm{~K}$} \\
& clean & complete \\
\hline$\leq-2.5$ & $2.180_{ \pm 0.088}^{ \pm 0.064}(0.093)$ & $2.164_{ \pm 0.090}^{ \pm 0.066}(0.053)$ \\
-2.5 to -2.0 & $2.253_{ \pm 0.058}^{ \pm 0.045}(0.075)$ & $2.247_{ \pm 0.057}^{ \pm 0.042}(0.075)$ \\
-2.0 to -1.5 & $2.326_{ \pm 0.187}^{ \pm 0.144}(0.059)$ & $2.326_{ \pm 0.187}^{ \pm 0.144}(0.059)$ \\
$\leq-1.5$ & $2.260_{ \pm 0.136}^{ \pm 0.098}(0.059)$ & $2.252_{ \pm 0.138}^{ \pm 0.099}(0.053)$ \\
\hline \multicolumn{3}{c}{$T_{\text {eff }} \geq 6000 \mathrm{~K}$} \\
\hline \multicolumn{3}{c}{ clean } \\
\hline$\leq-2.5$ & $2.180_{ \pm 0.088}^{ \pm 0.064}(0.093)$ & $2.180_{ \pm 0.088}^{ \pm 0.064}(0.093)$ \\
-2.5 to -2.0 & $2.232_{ \pm 0.046}^{ \pm 0.033}(0.075)$ & $2.227_{ \pm 0.043}^{ \pm 0.032}(0.075)$ \\
-2.0 to -1.5 & $2.362_{ \pm 0.126}^{ \pm 0.126}(0.059)$ & $2.362_{ \pm 0.176}^{ \pm 0.126}(0.059)$ \\
$\leq-1.5$ & $2.235_{ \pm 0.109}^{ \pm 0.077}(0.059)$ & $2.235_{ \pm 0.109}^{ \pm 0.077}(0.059)$ \\
\hline
\end{tabular}

be confirmed observationally. In the $[\mathrm{Fe} / \mathrm{H}]$ range between -2 and -1.5 , substantial lithium depletion relative to the plateau value sets in at $\sim 5600-5700 \mathrm{~K}$. Between 5700 and $5100 \mathrm{~K}$, the lithium depletion slope is $\sim 0.21$ dex per $100 \mathrm{~K}$.

\section{The lithium abundance in evolved stars}

We now address the case of more evolved stars. Their lithium abundance is plotted in Fig. 20 for four metallicity bins as a function of $T_{\text {eff }}$ which is now an indicator of the evolutionary status. We analyse the lithium behaviour in two separate groups of objects: turnoff and subgiant stars on one hand, and RGB stars on the other hand. We compare our results with the lithium data in globular clusters. 


\subsection{The mean lithium value in turnoff and subgiant stars}

As already mentioned in Sect. 10, we put a lower limit on the effective temperature $(5700$ and $6000 \mathrm{~K})$ for our analysis, this time in order to avoid any lithium depletion due to the first dredge-up. We consider stars both at the turnoff and on the subgiant branch as defined in Sect. 9.2. Two stars are excluded from the analysis, namely HIP 81276 $\left([\mathrm{Fe} / \mathrm{H}]=-1.50, T_{\text {eff }}=6523 \mathrm{~K}\right)$ of the clean sample and HIP $83320\left([\mathrm{Fe} / \mathrm{H}]=-2.56, T_{\text {eff }}=5984 \mathrm{~K}\right)$ of the $u b v y$ sample, because of their $A(\mathrm{Li})_{\mathrm{NLTE}}$ upper limits (of 1.705 and 1.287 respectively, see Fig. 20). The average value of $A(\mathrm{Li})_{\mathrm{NLTE}}$, the average and standard deviation and the root mean square of the observational errors are given in Table 13 for various metallicity and effective temperature intervals and for the clean and complete samples.

A very surprising result emerges here for the first time: Whatever the subsample we consider (i.e., clean or complete sample, $T_{\text {eff }} \geq 5700$ or $\left.6000 \mathrm{~K}\right)$, the mean value of $A(\mathrm{Li})_{\text {NLTE }}$ always appears to be higher for the subgiant stars than for the dwarfs, except for the most metal-deficient objects (i.e., with $[\mathrm{Fe} / \mathrm{H}] \leq-2.5)$ where the mean lithium abundance is very similar in both evolutionary statuses.

In the case of the evolved stars with $T_{\text {eff }} \geq 5700 \mathrm{~K}$ and $[\mathrm{Fe} / \mathrm{H}] \leq-1.5$,

$A(\mathrm{Li})_{\mathrm{NLTE}}=2.2599 \pm 0.0997$

and

$2.2524 \pm 0.0990$

for the clean and complete samples respectively. These values have to be compared respectively with $2.1768 \pm 0.0711$ and $2.1773 \pm 0.0840$ for the dwarfs the same range of effective temperature.

When we restrict our inspection to the stars with $T_{\text {eff }} \geq$ $6000 \mathrm{~K}$ and $[\mathrm{Fe} / \mathrm{H}] \leq-1.5$, the mean value of $A(\mathrm{Li})_{\mathrm{NLTE}}$ for the evolved stars is

$2.2349 \pm 0.0769$

for both the clean and the complete samples ${ }^{10}$, which has to be compared with $2.2154 \pm 0.0737$ and $2.2200 \pm 0.0740$ for the dwarf stars.

Once again, we note that our conclusions are not affected by having assumed -2.1 as the lower limit on the metallicity to be used in our colour- $T_{\text {eff }}$ calibration (see Sect. 5). The only effect of not considering this limit would be a slight increase of the Li mean value by $\sim 0.04-0.05$ dex.

Although the numbers for the dwarf and evolved stars are fully compatible within the quoted errors, the difference between the mean lithium values of both populations is stricking. As already discussed in Sect. 7.1, the dependence of the lithium abundance on gravity is weaker than that on effective temperature. At $T_{\text {eff }}=6000 \mathrm{~K}$ and $[\mathrm{Fe} / \mathrm{H}]=-1.5$, the typical effect is at most $+/-0.01$ dex in $A(\mathrm{Li})$ for $-/+1$ dex in $\log g$. We can thus see that even an error of 1 dex on the attributed

\footnotetext{
10 The $u b v y$ and $\beta$ samples do not contain additional objects.
}

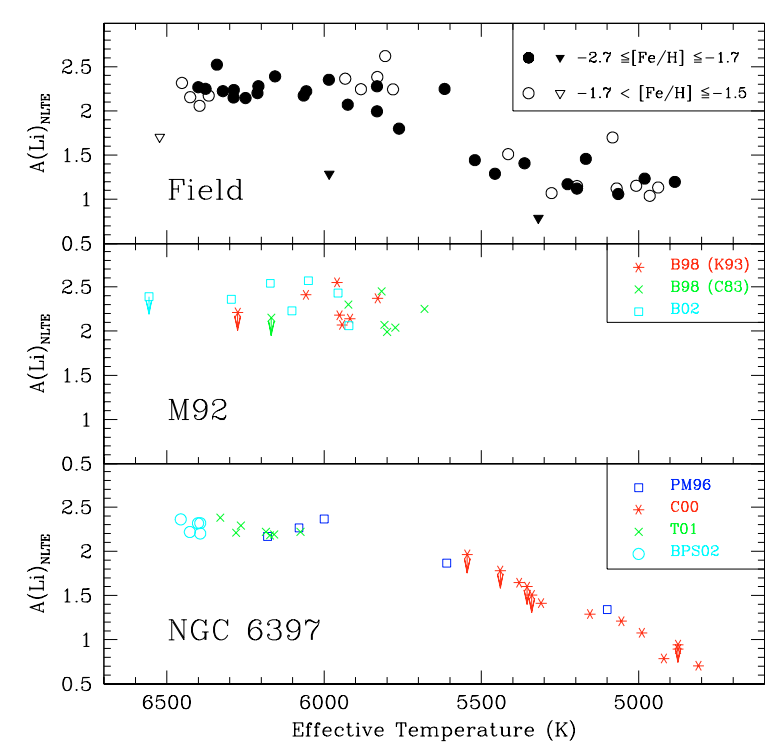

Fig. 21. Lithium abundance versus $T_{\text {eff }}$ for the turnoff and more evolved stars of our sample (upper panel, $T_{\mathrm{eff}}(2)$ ) and for their counterparts in the globular clusters M92 and NGC 6397 (lower and middle panel respectively). Effective temperature is here an indicator of the evolutionary status of the stars. For the field stars we focus on our sample stars in a limited metallicity range around that of the two globular clusters discussed here $([\mathrm{Fe} / \mathrm{H}]=-2.52$ for $\mathrm{M} 92$, King et al. 1998; $[\mathrm{Fe} / \mathrm{H}]=-2.02$ for NGC 6397, Thévenin et al. 2001). All the Li values are NLTE (see the text). For M 92, the original data are from Boesgaard et al. (1998, B98) and Bonifacio (2002, B02). For B98 study we plot the data both on the Carney (1983, C83) and King (1993, K93) temperature scales. The data for NGC 6397 are from Pasquini \& Molaro (1996, PM96), Castilho et al. (2000, C00), Thévenin et al. (2001, T01) and Bonifacio et al. (2002, BPS02).

gravity (which is very unlikely in view of the good precision of the Hipparcos parallax for most of the stars) cannot explain the difference on the mean lithium abundance that we obtain between the dwarf and subgiant stars. Neither can the dependence of our colour- $T_{\text {eff }}$ calibration on $\log g$. There is indeed a dependence for giant stars because $(b-y)$ measures the slope of the Paschen continuum, which in turn is affected by a change downwards of the gravity (cf. RM05b). However, what we call "evolved" stars are objects that have just passed the turn-off or that are located on the subgiant branch: they are not real giants, as defined in RM05b.

\subsection{Dispersion in evolved stars}

Our analysis reaveals another remarkable feature: Post-main sequence Population II stars appear to exhibit a non negligible Li dispersion. As can be seen for example in Fig. 20, this is already true at the turnoff and all along the evolution traced by the effective temperature.

For the subgiants near $6000 \mathrm{~K}$, PSB93 found a small spread in the lithium abundance around a mean value of 2.1. We see from the previously quoted numbers that the dispersion is actually not negligible in our sample of slightly evolved stars, independently of the adopted limit in effective temperature (5700 or $6000 \mathrm{~K}$ ). 
For the more evolved (i.e., cooler) stars, the lithium abundance is expected to decrease due to the first dredge-up at the effective temperature of $\sim 5700 \mathrm{~K}$, this dilution episode being completed around $5200 \mathrm{~K}$ (i.e., Deliyannis et al. 1990; Charbonnel 1995). This is what we observe, even though this region is not very well sampled. However if all the stars had left the main sequence with the same lithium content, they should share a common lithium abundance after the first dredge-up (remember that these stars have approximately the same initial mass). Instead, the evolved stars with $T_{\text {eff }}$ lower than $\sim 5500 \mathrm{~K}$ which have already undergone the first dredge-up dilution exhibit a relatively large lithium dispersion.

\subsection{Comparison with globular cluster stars}

The question of the lithium dispersion among metal-poor stars has already been discussed in the context of globular cluster studies. In Fig. 21 we show the data in the only two globular clusters for which $\mathrm{Li}$ abundances have been reported in stars down to the turnoff, namely M92 and NGC 6397 (middle and lower panel respectively). These are NLTE values, i.e. we corrected the LTE values reported in the literature for NLTE corrections which were computed following the same prescriptions we used for our field stars sample. Unfortunately, the unavailability of Strömgren photometry for all the globular cluster stars analysed in M92 and NGC 6397 did not allow us to re-derive their lithium abundances based on our temperature scale. For M92 we rely on two analyses: i) Boesgaard et al. (1998, hereafter B98), from which we report the $\mathrm{Li}$ values for both temperature scales (Carney 1983, C83; and King 1993, K93) discussed in their original paper; ii) Bonifacio (2002, hereafter B02) which is based on B98 equivalent widths and on the temperature calibration by Bonifacio et al. (2002, hereafter BSP02). For NGC 6397, the Li abundances are taken from Pasquini \& Molaro (1996), Castilho et al. (2000), Thévenin et al. (2001) and Bonifacio et al. (2002, BSP02). In Fig. 21 the globular cluster data are compared with the NLTE Li abundances of our evolved field stars in the metallicity range around that of the clusters $(-2.7 \leq[\mathrm{Fe} / \mathrm{H}] \leq-1.7$, black symbols in the upper panel).

B98 investigated the lithium abundance in seven stars near the turnoff of the old and metal-poor cluster M92 $([\mathrm{Fe} / \mathrm{H}]=-2.52$, King et al. 1998). They reported a dispersion of a factor of $\sim 2.6$ for the subgiant stars in a region around $T_{\text {eff }} \sim 5800 \mathrm{~K}$ or $5950 \mathrm{~K}$ (depending on the adopted temperature scale, C83 or K93 respectively) as can be seen in the middle panel of Fig. 21. Contrary to B98, B02 concluded that there is no strong evidence for intrinsic dispersion in $\mathrm{Li}$ abundances among the M92 stars, although a dispersion as large as 0.18 dex is possible. B02 actually warned the reader that no definitive conclusion can be drawn on the intrinsic dispersion in this stellar cluster on the basis of the currently available spectra. Better observations with higher $S / N$ ratios are still needed for these very faint M 92 stars.

Although the data points are relatively scarce in this region, one sees from Fig. 21 that some dispersion indeed exists among field stars in the $T_{\text {eff }}$ range of B98's data. The

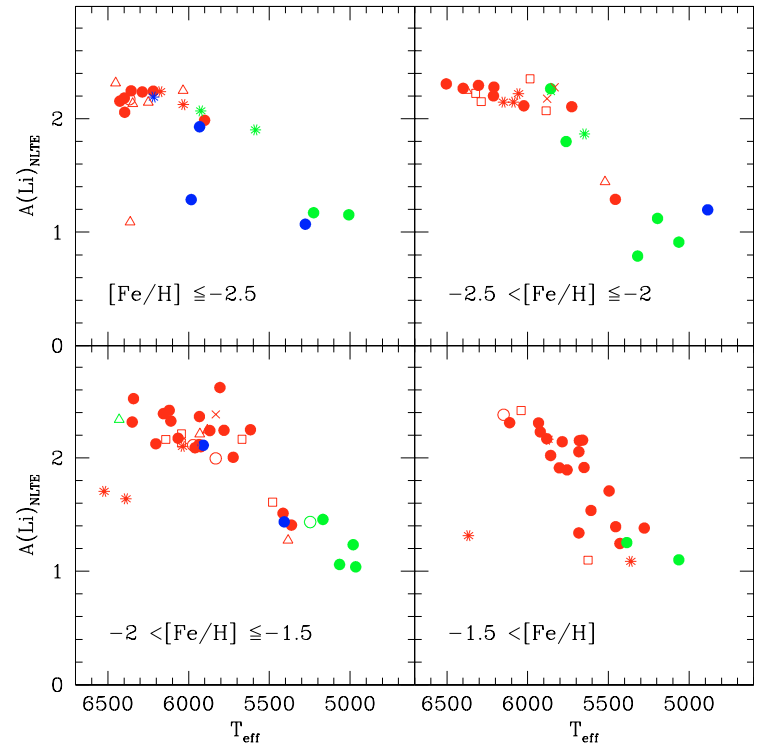

Fig. 22. Duplicity and variability among our complete sample. Asterisks: confirmed single- or double-lined binaries (Latham et al. 2002; Carney et al. 2003, 1994). Open squares: stars marked as spectroscopic binaries in Bonifacio \& Molaro (1997). Open triangles: suspected binaries (Latham et al. 2002). Crosses: binaries or stars in double/multiple system as reported in SIMBAD. Filled circles: single stars. Open circles: variable stars as reported in SIMBAD.

stars HIP 36430, 92775 and 102718 which have respectively $[\mathrm{Fe} / \mathrm{H}]=-2.10,-2.18$ and $-1.80, T_{\text {eff }}=5985,5762$ and $5832 \mathrm{~K}$, and very similar $\log \left(L / L_{\odot}\right)=1.69 \pm 1.71,1.88 \pm 0.79$ and $1.71 \pm 2.27$, present significant differences in their NLTE-Li abundance: $2.352 \pm 0.068,1.799 \pm 0.113$ and $1.995 \pm 0.165$. This corresponds to the dispersion claimed by B98 in M92. In addition at approximatively the same effective temperature the slightly more metal-poor star HIP $83320([\mathrm{Fe} / \mathrm{H}]=-2.56$, $\left.T_{\text {eff }}=5984 \mathrm{~K}, \log \left(L / L_{\odot}\right)=0.77 \pm 1.17\right)$ shows only an upper limit for $\mathrm{Li}(A(\mathrm{Li}) \leq 1.287)^{11}$. B98 discussed the case of the halo subgiant $\mathrm{BD}+23^{\circ} 3912$ (HIP 99423) which was found by King et al. (1996) to have a remarkably high lithium abundance of $2.56 \pm 0.07$. This star is also in our sample, with the following parameters: $[\mathrm{Fe} / \mathrm{H}]=-1.54, T_{\text {eff }}=5806 \mathrm{~K}$, and $\log \left(L / L_{\odot}\right)=0.48 \pm 0.12$. We find a relatively high NLTE $\mathrm{Li}$ abundance of 2.620 for this object. Although its relatively high metallicity makes this star not very relevant when compared to M 92 stars, we think that it reinforces the case for dispersion.

The lithium behavior in NGC $6397([\mathrm{Fe} / \mathrm{H}]=-2.02$, Thévenin et al. 2001) is instead completely different: stars with $T_{\text {eff }} \geq 6000 \mathrm{~K}$ share the same lithium abundance with essentially no intrinsic scatter. No lithium abundance has been reported up to now in this cluster for stars over the $\sim 5600$ to $6000 \mathrm{~K}$ range. Observations in this region would be useful.

11 We note that the error on the Hipparcos parallax is relatively high for HIP 36430, 92775 and 83320, which turns into a significant error bar on the determined luminosities. The status of subgiant can however be attributed relatively safely to these three objects (see also Fig. 16). 
Table 14. Average value of $A(\mathrm{Li})_{\mathrm{NLTE}}$ and average deviation for $[\mathrm{Fe} / \mathrm{H}] \leq-1.5$ : When all the stars are considered regardless of their possible duplicity or variability (Col. a), when all the suspected and confirmed binaries are omitted (Col. b), and when only the confirmed binaries are excluded (Col. c). In cases (b) and (c) the stars reported as variable in SIMBAD are not considered. The first and second lines contain the values for the clean and the complete samples respectively.

\begin{tabular}{|c|c|c|c|}
\hline & (a) & (b) & (c) \\
\hline \multicolumn{4}{|c|}{ Dwarfs } \\
\hline$T_{\text {eff }}$ & $2.177 \pm 0.071$ & $2.184 \pm 0.098$ & $2.186 \pm 0.092$ \\
\hline$\leq 5700 \mathrm{~K}$ & $2.177 \pm 0.084$ & $2.176 \pm 0.108$ & $2.185 \pm 0.102$ \\
\hline$T_{\text {eff }}$ & $2.215 \pm 0.074$ & $2.243 \pm 0.076$ & $2.235 \pm 0.075$ \\
\hline$\leq 6000 \mathrm{~K}$ & $2.220 \pm 0.074$ & $2.257 \pm 0.075$ & $2.253 \pm 0.074$ \\
\hline \multicolumn{4}{|c|}{ turnoff and subgiant stars } \\
\hline$T_{\text {eff }}$ & $2.260 \pm 0.098$ & $2.297 \pm 0.119$ & $2.307 \pm 0.127$ \\
\hline$\leq 5700 \mathrm{~K}$ & $2.252 \pm 0.099$ & $2.301 \pm 0.126$ & $2.284 \pm 0.106$ \\
\hline$T_{\text {eff }}$ & $2.235 \pm 0.077$ & $2.260 \pm 0.105$ & $2.289 \pm 0.129$ \\
\hline$\leq 6000 \mathrm{~K}$ & $2.235 \pm 0.077$ & $2.260 \pm 0.105$ & $2.253 \pm 0.085$ \\
\hline
\end{tabular}

\section{Duplicity and variability}

After a careful inspection of the literature in order to identify single- and double-lined binaries (we mainly used the extensive surveys and listings by Latham et al. 2002; Carney et al. 1994, 2003) we then tested if any of our above conclusions on the mean lithium abundance and on the dispersion may have been affected by the inclusion of binary stars. This is especially important because the literature-based $E W$ values may have been derived at a time when the binary nature of the star was still unknown. In order to get the most conservative answer, we also checked the SIMBAD database for binary and variable stars, and we included also the stars marked as "binaries" in BM97. All the stars thus identified are marked with different symbols in Table 6 (2nd column, see the legend at the bottom of the version of the table provided on-line) and the situation can be visualized in Fig. 22. Over the whole metallicity and effective temperature ranges sampled in this paper, $27(10,3)$ stars from the clean sample are confirmed binaries (suspected binaries, variable stars). The corresponding numbers for the $u b v y$ and $\beta$ samples are $1(0,0)$ and $4(1,3)$.

We compute the average value of $A(\mathrm{Li})_{\mathrm{NLTE}}$ and the average deviation of the observational errors for two cases: We first omit all the suspected and confirmed binaries, and then we exclude only the confirmed binaries. In both cases, the stars reported as variable in SIMBAD are not considered.

The results are reported in Table 14 for the dwarf stars on one hand and for the turnoff and subgiant stars together on the other hand. In each case the first and second lines refer respectively to the values of the clean and complete samples. We also recall the values discussed previously, which were obtained regardless of the duplicity or variability of the stars (see Col. a).

First, we see that in all the considered cases, the absolute numbers for the mean lithium abundance are always slightly higher (although fully compatible within the errors) when
Table 15. Sample stars with Li upper limits. The stars with a $*$ lie in the ranges in effective temperature and metallicity chosen to delimitate the Li plateau. The others are either cooler or more metal-rich. The \# indicates the stars for which a $v$ sini value higher than $4.5 \mathrm{~km} \mathrm{~s}^{-1}$ could be derived by Ryan et al. (2002) or Ryan \& Elliott (2004). See the text for more details.

\begin{tabular}{|c|c|c|c|c|c|c|c|}
\hline HIP & & & $T_{\text {eff }}$ & $\log L / L_{\odot}$ & {$[\mathrm{Fe} / \mathrm{H}]$} & Li u.l. & $\begin{array}{l}\text { single } \\
\text { /binary }\end{array}$ \\
\hline \multicolumn{8}{|l|}{ Dwarfs } \\
\hline 72561 & $*$ & $\#$ & 6388 & $-0.12 \pm 0.30$ & -1.66 & 1.639 & $\mathrm{~b}$ \\
\hline 100682 & $*$ & $\#$ & 6362 & $-0.12 \pm 0.20$ & -2.83 & 1.088 & $\mathrm{~b}$ \\
\hline 67655 & & & 5429 & $-0.38 \pm 0.04$ & -1.03 & 1.245 & $\mathrm{~s}$ \\
\hline 67863 & & & 5683 & $-0.04 \pm 0.04$ & -0.88 & 1.338 & $\mathrm{~s}$ \\
\hline \multicolumn{8}{|c|}{ Subgiants } \\
\hline 81276 & * & $\#$ & 6523 & $0.76 \pm 0.58$ & -1.50 & 1.705 & $\mathrm{~b}$ \\
\hline 83320 & * & \# & 5984 & $0.71 \pm 0.77$ & -2.56 & 1.265 & $\mathrm{~s}$ \\
\hline 55022 & & $\#$ & 6367 & $0.50 \pm 0.14$ & -1.29 & 1.313 & s \\
\hline 60719 & & & 5319 & $1.44 \pm 0.21$ & -2.32 & 0.789 & s \\
\hline
\end{tabular}

binary and variable stars are excluded. Secondly, the dispersion increases but only slightly. This confirms the early finding by Molaro (1991) that known binaries do not exhibit lithium abundances significantly different than the other stars and that they do not introduce significant scatter into the plateau. Finally, the finding that plateau dwarf stars exhibit a lower lithium mean value (although fully compatible within the quoted errors) than their evolved counterparts is robust and resists the duplicity check.

\section{Stars with extreme Li abundances}

\subsection{Plateau stars with Li upper limits}

In all the above discussions we have quoted the values for the mean lithium abundance and dispersion as well as for the trends with effective temperature and metallicity obtained without taking into account the stars with $\mathrm{Li}$ upper limits. The main characteristics of these Li-depleted stars are summarized in Table 15 . In the metallicity and effective temperature ranges we have chosen to delimit the plateau, we have two dwarfs (HIP 72561 and 100682) and two more evolved stars (HIP 81276 and 83320). The first 3 of these objects have relatively high effective temperatures and are either dwarfs or subgiants lying very close to the turnoff, while the last one is clearly crossing the Hertzsprung gap.

Several studies have been devoted to these so-called ultralithium-deficient (hereafter ULDs) halo stars ${ }^{12}$. Understanding their nature is crucial. They should indeed be excluded from the investigations on the primordial $\mathrm{Li}$ abundance if they belong to

\footnotetext{
12 Additional ULDs which can be found in the literature do not have Hipparcos parallaxes and are thus excluded from our sample and from the present discussion. See references in Ryan et al. (2002).
} 
a very special class of objects. However if they appear to be the extreme representatives of a process that has affected all the stars along the plateau, they would be precious clues on Li depletion in halo stars. Up to now, no mechanism has been unambiguously identified as responsible for the ULD phenomenon.

Norris et al. (1997) looked at the abundances of many elements in the spectra of HIP 72561, 83320 and 100682 and found no common abundance anomaly that could be associated with the Li deficiency (see also Ryan et al. 1998; and Ryan \& Elliot 2004). HIP 100682 presents an overabundance of the heavy-neutron capture elements (a feature which is shared by $\sim 25 \%$ of halo objects with similar $[\mathrm{Fe} / \mathrm{H}])$ whereas none of the other two ULDs shows such an enrichment.

Hobbs et al. (1991) and Thorburn (1992) suggested that HIP 83320 and 100682 might be the progeny of blue stragglers that had depleted their Li to undetectable levels earlier on and are now evolving redwards, through the temperature range of the plateau. However Thorburn noticed that there are too many ULDs compared to the number of known halo blue stragglers.

The possible role of duplicity in producing the observed Li depletion in the ULDs via mass transfer has been discussed several times in the literature. Ryan et al. (2001a) proposed that the same mass transfer processes responsible for the field blue stragglers should also produce sub-turnoff-mass objects which would be indistinguishable from normal stars except for their $\mathrm{Li}$ abundance. This could in principle lead to Li depletion and Ba enhancement as observed in HIP 100682 if the donor companion was an AGB star. On the other hand, there also exist the possibility that the mass transfer from an RGB or an AGB star is not always accompanied by other abundance anomalies. HIP 72561 and 81276 appear to be multiple systems and HIP 100682 is a suspected binary (Carney et al. 1994, 2003; Norris et al. 1997). HIP 83320 however is a single star.

Ryan et al. (2002, hereafter R02) looked in detail at a sample of 18 halo main-sequence turnoff stars including 4 ULDs $^{13}$. They discovered that 3 out of these 4 Li-depleted stars (but none of the Li-normal stars) exhibited unusually broad absorbtion lines that could be attributed to rotational broadening. Among these objects are HIP 72561 and 81276 for which values of $5.5 \pm 0.6$ and $8.3 \pm 0.4 \mathrm{~km} \mathrm{~s}^{-1}$ were respectively inferred for $v \sin i$ (Note that the "Li-normal" stars have undetectable rotation, generally below $3 \mathrm{~km} \mathrm{~s}^{-1}$ ). The 3 broadened objects of R02's sample all have relatively high effective temperature and they lie close to the turnoff. Furthermore, they are all confirmed binaries with orbits that are not tidally synchronised. These complementary features lead $\mathrm{R} 02^{14}$ to draw a connection between Li depletion, rapid rotation and mass and angular momentum transfer from a companion, now the white dwarf remnant of a star initially more massive than the present plateau stars, as confirmed by the projected companion masses inferred by Carney et al. (2001). Ryan \& Elliott (2004) looked again at

\footnotetext{
13 Two of these objects do not appear in our study, one of them (CD-31 $\left.{ }^{\circ} 19466\right)$ because it does not have an Hipparcos parallax, the other because of its relatively high metallicity $\left(\mathrm{BD}+51^{\circ} 1817\right.$, $[\mathrm{Fe} / \mathrm{H}]=-0.88)$.

14 This followed Fuhrmann \& Bernkopf (1999) who suggested such a relation in the case of thick-disk, binary blue stragglers.
}

line-broadening in ULDs and showed that 5 out of 8 have rotation velocities in excess of $4 \mathrm{~km} \mathrm{~s}^{-1}$ and that 4 out of 5 are confirmed binaries. The invoked mass transfer thus has not resulted in the merger of the components.

In the scenario proposed by Ryan and collaborators, the Li depletion could be due either to mixing triggered during the accretion event, or to Li deficiency of the donor only or of both companions prior to the mass transfer. If additional evidence could be found in favor of this scenario for the formation of ULDs, it would become clear that these objects are not useful to infer the primordial abundance nor to constrain the classical depletion mechanism(s). However, although this explanation is appealing and may work for the line-broadened and hot ULD stars, the unbroadened and/or single ULDs still await a plausible interpretation. As we shall discuss in more detail later on, the fact that all these stars lie on or originate from the hottest side of the plateau may reveal an alternative explanation.

\subsection{Li upper limits outside the plateau range}

In our sample some other stars that are cooler and/or more metal-rich than the plateau also have Li upper limits, namely the dwarfs HIP 67655 and 67863 and the post-turnoff stars HIP 55022 and 60719 (see Table 15).

HIP 55022 is a relatively hot and metal-rich subgiant that lies very close to the turnoff. It is a confirmed binary, and could be the high-metallicity counterpart of the ULDs previously described. Ryan \& Elliott (2004) give a $v \sin i$ value of $10.4 \pm 0.2 \mathrm{~km} \mathrm{~s}^{-1}$ for this star.

All the other three objects are single stars. HIP 67655 and 67863 are cool dwarf stars that lie in the effective temperature region where the lithium depletion relative to the plateau is strong (see Sect. 10.5). Therefore, the non-detection of $\mathrm{Li}$ in these two stars does not appear to be a real abnormalty.

HIP 60719 is a single star at the base of the RGB that is undergoing Li dilution. Although this could explain its Li upper limit, this star clearly lies below the other evolved stars of our sample indicating that it has undergone a more severe lithium depletion. It is slightly more evolved than the single subgiant HIP 83320 which was discussed previously. For none of these two stars can mass transfer be advocated to explain the Li behavior. Note that HIP 60719 and 83320 additionally contribute to the Li dispersion observed in evolved stars and discussed in Sect. 11.2.

\subsection{Stars with abnormally high Li abundances}

On the other extreme, some stars of our sample present relatively high lithium abundances, namely HIP 86694 and 99423. Both of them are post-turnoff stars. We have tried to identify in our sample other stars that share the same characteristics as these objects, but differ in their Li abundance.

No real pair star could be attributed to HIP 86694. This star appears in several independent studies which all confirm its high $\mathrm{Li}$ content. $A(\mathrm{Li})_{\mathrm{NLTE}}$ values between 2.481 and 2.554 are obtained on the basis of the extreme literature $E W$ determinations. 
HIP 99423 has the same characteristics as HIP 73385 and 114962 (Teff of 5806, 5831, 5883 K; [Fe/H] of -1.54 , $-1.62,-1.52 ; \log L / L_{\odot}$ of $\left.0.482,0.552,0.554\right)$ but a significantly higher Li abundance (2.620, 2.383 and 2.245). Although HIP 99423 seems to be a single star, its "pairs" are suspected or confirmed binaries.

\subsection{Clues for another Li history in stars originating from the hot side of the plateau?}

We summarise our findings concerning the stars that present $\mathrm{Li}$ abnormalities, i.e., Li deficiencies or high $\mathrm{Li}$ values:

- Except for HIP 67655 and 67863 which are cool dwarf stars lying in the effective temperature range where substantial lithium depletion is expected to occur, all the other stars of our sample for which only an upper limit for Li could be derived are either

1. dwarf or turnoff stars at the extreme hot end of the plateau; or

2. slightly more massive subgiants that have evolved from this blue region.

- The stars with abnormally high Li are all post-turnoff stars.

In other words, all these objects lie on or originate from the hot side of the plateau, and are thus more massive than the plateau dwarf stars for which no Li dispersion nor any other anomalies have been detected. This has to be related to the non-negligible lithium dispersion and the higher lithium mean value that we get for post-main sequence stars compared to plateau dwarfs in a given range in effective temperature (see Sect. 11). It is thus tempting to suggest that the most massive of the halo stars still observable exhibit a Li dispersion together with some "extreme" Li behavior (i.e., Li over-depletion or Li preservation) which reflect a different Li history to that of the less massive plateau dwarf stars. We will come back to this crucial point in Sect. 14.

\subsection{Stars with ${ }^{6}$ Li detection}

Although we do not aim to homogeneize the ${ }^{6} \mathrm{Li} /{ }^{7} \mathrm{Li}$ data available in the literature, we need to comment on the very few halo stars in which ${ }^{6} \mathrm{Li}$ has been detected. This isotope is extremely difficult to observe, being only a weak component, blending with the much stronger ${ }^{7} \mathrm{Li}$ doublet at $670.7 \mathrm{~nm}$. The isotopic separation is $0.16 \AA$ only. So far it has been detected in only a few stars which all belong to our sample, namely HIP 8572 (G271-162), HIP 48152 (HD 84937) and HIP 96115 (BD + $26^{\circ} 3578$ ). For all the other halo stars that have been looked at, only upper limits could be derived (see Smith et al. 1993, 1998, 2001; Hobbs \& Thorburn 1994, 1997; Cayrel et al. 1999a; Hobbs et al. 1999; Nissen et al. 2000).

In Table 16 we recall the main characteristics of our sample stars for which positive detections of ${ }^{6} \mathrm{Li}$ have been reported and published. Note that the 3 stars under scrutiny are at the turnoff (i.e., with relatively high $\log T_{\text {eff }}$ ) and have relatively
Table 16. Sample stars with published ${ }^{6} \mathrm{Li}$ detection. The data are from Smith et al. (1998, sln98), Cayrel et al. (1999a, c99) and Nissen et al. (2000, n00). Columns 2 and 3 give the $T_{\text {eff }}(2)$ and $[\mathrm{Fe} / \mathrm{H}]$ values that we attributed to the stars while Cols. 4 and 5 display the values quoted in the original papers. For completeness, we note that a re-analysis by Asplund et al. (2005) of HIP 8572 and HIP 96115 from very high resolution and $S / N$ UVES (Dekker et al. 1999) spectra finds a slightly less than $2 \sigma$ detection for HIP 8572 and no ${ }^{6} \mathrm{Li}$ detection for HIP 96115.

\begin{tabular}{cccccccc}
\hline \hline \multirow{2}{*}{$\mathrm{HIP}$} & $T_{\text {eff }}$ & {$[\mathrm{Fe} / \mathrm{H}]$} & $T_{\text {eff }}$ & {$[\mathrm{Fe} / \mathrm{H}]$} & $\mathrm{Li}$ & ${ }^{6} \mathrm{Li} /{ }^{7} \mathrm{Li}$ & Ref \\
& $(2)$ & & lit & lit & NLTE & & \\
\hline 8572 & 6287 & -2.51 & 6295 & -2.15 & 2.236 & $0.02 \pm 0.01$ & n00 \\
48152 & 6377 & -2.28 & 6300 & -2.30 & 2.249 & $0.05 \pm 0.02$ & $\mathrm{c} 99$ \\
& & & 6300 & -2.25 & & $0.06 \pm 0.02$ & $\mathrm{n} 00$ \\
96115 & 6322 & -2.42 & 6280 & -2.60 & 2.223 & $0.05 \pm 0.03$ & $\sin 98$ \\
\hline
\end{tabular}

low $[\mathrm{Fe} / \mathrm{H}]$ values ${ }^{15}$. They have no additional peculiarities except for ${ }^{6} \mathrm{Li}$ when compared to other stars in which this isotope has been looked for but not detected (see e.g. Smith et al. 1998).

Additionally, recent results from Asplund et al. (2005) indicate nine new ${ }^{6} \mathrm{Li}$ detections, in stars spanning a range in metallicity from $[\mathrm{Fe} / \mathrm{H}]=-1.3$ down to -2.7 . Four out of these nine objects belong to our sample and again turn out to be stars with relatively high effective temperatures, close to the turnoff, and with relatively low metallicity.

${ }^{6} \mathrm{Li}$, together with ${ }^{9} \mathrm{Be}$, and ${ }^{10} \mathrm{~B}$ (the exact contribution from $v$-spallation in supernovae to ${ }^{11} \mathrm{~B}$ being still under debate), is believed to originate primarily (plus some extra contribution by $\alpha-\alpha$ reactions and other stellar sources, the latter important at higher metallicites) from spallation reactions in the interstellar medium between cosmic-ray (CR) $\alpha$-particles and protons and heavy nuclei like CNO (Reeves et al. 1973) . Basic considerations about the production rate and environment of these light nuclides predict a quadratic slope in the logarithmic plane ((light nuclide, e.g. $\left.{ }^{6} \mathrm{Li}\right)$,metallicity), whereas the almost linear slope derived from spectroscopic analyses of Be and B abundances in stars of the Galactic halo (e.g. Boesgaard et al. 1999 for Be, Primas et al. 1999 for B) seems instead to indicate a primary (instead of secondary) origin, which in turn requires the need for a production mechanism independent of the metallicity in the ISM. One can generally refer to the two abovementioned scenarios as to classical vs. reverse spallation reactions, with the latter having several implementations based on different assumptions.

Despite the small existing number of ${ }^{6} \mathrm{Li}$ detections, this light nuclide seems to have a different history, showing a much flatter evolution (i.e. most of GCR-based models underproduce the amount of ${ }^{6} \mathrm{Li}$ observed in halo stars).

\footnotetext{
15 Standard models of Pop II stars predict only a slight depletion of ${ }^{6} \mathrm{Li}$ (which occurs mainly during the pre-main sequence phase) for stars which are now at the turnoff. The depletion factor increases for lower mass dwarf stars (see e.g. Deliyannis \& Malaney 1995; and Cayrel et al. 1999b). This explains why ${ }^{6} \mathrm{Li}$ has been found so far only in turnoff stars relatively metal-poor.
} 
Because ${ }^{6} \mathrm{Li}$ is the most fragile isotope to proton destruction, its presence in the atmosphere of Pop II stars is usually considered as a very severe limit on the amount of ${ }^{7} \mathrm{Li}$ depletion. This argument is very often used in favor of the Li plateau abundance being the primordial value (Brown \& Schramm 1988), a conclusion that is challenged now by the $\mathrm{CMB}$ constraint. In this context several questions remain open. First, what is the pre-stellar value of ${ }^{6} \mathrm{Li} /{ }^{7} \mathrm{Li}$ (Probably not the one we observe now, because of the different sensitivity of both isotopes to nuclear destruction)? How can we explain the presence of ${ }^{6} \mathrm{Li}$ in some of the plateau stars? Why do some turnoff stars exhibit some ${ }^{6} \mathrm{Li}$ in their spectra while the majority does not?

One possibility calls for some ${ }^{6} \mathrm{Li}$ production at the stellar surface by either stellar flares (Deliyannis \& Malaney 1995, but see the criticism by Lemoine et al. 1997) or Galactic cosmic rays (Lambert 1995). The other possibility is more related to the questions about the origin of ${ }^{6} \mathrm{Li}$ in metal-poor stars and about a possible scatter in the ${ }^{6} \mathrm{Li} /{ }^{7} \mathrm{Li}$ ratio in the ISM at a given metallicity. The type of cosmic ray sources and the production mechanims operating in the early, forming galaxy are still very controversial (see e.g. Vangioni-Flam et al. 1999). Some models for the formation of light elements by cosmic ray processes predict a scatter of one order of magnitude in the abundances of ${ }^{6} \mathrm{Li}, \mathrm{Be}$ and $\mathrm{B}$ relative to $\mathrm{Fe}$. This is the case of the bimodal superbubble model by Parizot \& Drury (1999) and of the supernovae-driven chemical evolution model for the Galactic halo by Suzuki et al. (1999). The superbubble scenario is actually challenged by several observations, for example the fact that there seems to exist no association of core collapse SN with superbubbles but rather to HII regions which reflect the metallicity of the ambiant interstellar medium rather than that of the SN (see the discussion in Prantzos 2004).

Suzuki \& Inoue (2004) recently presented an additional mechanism for cosmic ray production of ${ }^{6} \mathrm{Li}$ by virialisation shocks during hierarchical structure formation of the Galaxy. In their model, cosmic rays accelerated by this source dominate the production of ${ }^{6} \mathrm{Li}$ (compared to $\mathrm{SNe}$ as the main source of acceleration), without co-producing $\mathrm{Be}$ and $\mathrm{B}$. This seems to better account for the observed ${ }^{6} \mathrm{Li}$ data in halo stars, and in particular for the "plateau" of ${ }^{6} \mathrm{Li} / \mathrm{H}$ reported by Asplund et al. (2005), than the SN-driven cosmic ray scenarios. Large ${ }^{6} \mathrm{Li}$ scatter should be a natural consequence of this process, a higher initial content being expected in stars belonging to the inner halo compared to those of the outer halo. This is an attractive scenario, although very difficult to estimate quantitatively due to the many uncertainties on the physics of structure formation and on the energetics of the implied cosmic rays.

Clearly the detection of ${ }^{6} \mathrm{Li}$ in low metallicity halo stars constitutes a challenge to cosmic ray spallation and/or stellar mixing along the plateau. Alternatively it may be the case that the lithium isotope ratio probes Physics beyond the Standard Model. Indeed Jedamzik (2004) found that the decay of a supersymetric particle like the gravitino or neutralino around $10^{3} \mathrm{~s}$ after the Big Bang may at the same time yield to a reduction of the primordial ${ }^{7} \mathrm{Li} / \mathrm{H}$ by a factor $2-3$ and produce ${ }^{6} \mathrm{Li}$ to the magnitude observed in halo stars. However before we turn to such possibilities, the astrophysical uncertainties and solutions must be critically assessed.

\section{Implications for stellar structure and evolution}

Since the discovery of lithium in Pop II stars, not only the observers have been very active on the subject. Theoreticians, too, have taken advantage of the lithium diagnostic to probe the interior and the evolution of Pop II stars. Although these low-mass objects seem to be very simple at first sight, their Li behavior is paradoxal and has still not been fully understood. Part of the difficulty comes from the fact that nothing is known about the initial conditions of an important quantity like the angular momentum distribution. We do not aim here to discuss all the literature published on the subject (see the review by Pinsonneault et al. 2000 and Talon \& Charbonnel 2004, for more recent references). We rather use the specific findings of our analysis to propose a synthesis, i.e., to extract from the theoretical debate some of the most adapted and promising directions that require further investigation.

The (incorrectly) so-called "standard" stellar models ${ }^{16}$ have long been very popular in the plateau debate, mainly because they predict no variation of the surface $\mathrm{Li}$ abundance with time and consequently no Li dispersion from star to star along the plateau. The corresponding $\mathrm{Li}$ isochrones thus naturally support the conclusion that the Spite plateau reflects the cosmological $\mathrm{Li}$ abundance. This belief has been shaken by the recent CMB measurements.

In addition to and maybe even more fundamental (from the stellar physicist point of view) than the CMB result, the validity of these stellar models can be refuted on the basis of some of their assumptions, the most critical of which being the absence of transport of chemicals except in the convection zones. This hypothesis denies the fundamental nature of a star which is a gaseous mixture of elements with various atomic masses. As a result the stellar gas cannot be in equilibrium as a whole until each component reaches its own equilibrium via gravitational separation and thermal diffusion (Eddington 1929). Thanks to helioseismology it is now fully recognised that atomic diffusion must be an integral part of stellar evolution computations and in particular of the standard model ${ }^{17}$ of the Sun (i.e., Richard et al. 1996; Bahcall et al. 1997) and of low-mass stars.

The relative unpopularity of the Pop II standard models rests mainly on the fact that pure atomic diffusion leads to a degree of surface $\mathrm{Li}$ depletion which increases with effective temperature along the plateau (Michaud et al. 1984; Deliyannis et al. 1990; Proffitt \& Michaud 1991; Chaboyer \& Demarque 1994; Vauclair \& Charbonnel 1995; Salaris \& Weiss 2001; Richard et al. 2002). As confirmed again in the present analysis, this feature is not observed, although some of the hottest dwarf

\footnotetext{
16 This refers to the modeling of non-rotating, non-magnetic stars in which convection is the only transport process considered.

17 It is now widely accepted that the standard stellar models are those in which the effects of atomic diffusion are taken into account and not counterbalanced by any macroscopic process. They are calculated from first principles without any arbitrary parameter except for the mixing length.
} 
stars with Li deficiency may exhibit the effects of atomic diffusion (see Sect. 13.1, and discussion below). This difficulty can be simply read as the signature of some macroscopic processes that minimize the effects of atomic diffusion which is always present in stellar interiors and cannot be arbitrarily turned off. Such a suggestion was already made in a more general context by Eddington (1929) who pointed out that some mixing must occur in the stellar radiative zone in order to prevent the gravitational settling and the thermal diffusion of heavy elements, the effects of which were not always observed. Consistently, such a process is also required in Pop II stars to counteract the settling of heavier elements in order to explain the close similarity of iron abundances in near turnoff, sub-giant and lower RGB stars in globular clusters, and to reproduce the observed morphologies of globular cluster color-magnitude diagrams (see VandenBerg et al. 2002).

Several processes have been invoked to counteract atomic diffusion in stellar interiors. In the case of Pop II stars the possible candidates that have been studied (in models including or not atomic diffusion) are : Rotation (Vauclair 1988; Chaboyer \& Demarque 1994; Pinsonneault et al. 1991, 1999, 2002; Vauclair \& Charbonnel 1995), stellar wind (Vauclair \& Charbonnel 1995), interaction between meridional circulation and helium settling (Théado \& Vauclair 2001). All these models have real difficulties, unless some adhoc assumptions are made, in reconciling a non-negligible Li destruction with both the flatness and the extremely small dispersion on the plateau as definitively needed in view of the difference between the WMAP constraint and the plateau value discussed in this paper. This does not mean of course that the physical processes invoked are not at work in Pop II stars but it indicates at least that their theoretical description is still incorrect or incomplete.

Richard et al. (2002, hereafter Ri02, 2004) re-investigated the case of the Li plateau with a new generation of Pop II models that include self-consistently all the effects of atomic diffusion in the presence of weak turbulence whose nature is not postulated a priori. They discuss in great detail the parametrization of turbulence that has to be included in order to reproduce the constancy of the Li plateau (see also Proffitt \& Michaud 1991 and Vauclair \& Charbonnel 1998 and references therein). The mean $\mathrm{Li}$ value we derive in the present paper for the plateau dwarf stars favors such a model with an intermediate efficiency of turbulence (actually between the so-called T6.0 and T6.25 models of Ri02 ${ }^{18}$ ). In addition Ri02 show that acceptable variations in the turbulence (i.e., from no macroscopic motion to that needed to fit the plateau) can lead to variations of the $\mathrm{Li}$ abundance as high as $0.5-0.6 \mathrm{dex}$ at the turnoff. As can be seen in their Figs. 14 and 16 the Li abundance at that evolutionary stage is indeed a very sensitive function of the exact position in the HR diagram and of the adopted turbulence. The abundance variations and in particular the Li deficiencies that we find in our data at or just after the turnoff as well as those found by B98 in M92 are similar to those expected at this evolutionary stage by Ri02 in the case of variations of turbulence from star to star. However in Ri02's models these abundance

\footnotetext{
18 In a $T x . y$ model, the turbulent diffusion coefficient is 400 times larger than the He atomic diffusion at $\log T=x . y$ and varies as $\rho^{-3}$.
}

variations are theoretically erased by dilution in the subgiants when they reach $\sim 6000 \mathrm{~K}$, although the dispersion persists in the data at and below this effective temperature. This difficulty may be alleviated by assuming that some stars have undergone even stronger turbulence that lead to stronger Li destruction.

As previously mentioned, Richard and collaborators do not postulate the physical mechanism that causes the turbulence required by their models. Our present results bring a very important piece to the puzzle. We could show that the turnoff and more evolved Pop II stars present a slightly higher Li mean value as well as a larger Li dispersion than the less massive dwarfs. This points towards a mechanism, the efficiency of which changes as one reaches the extreme blue edge of the Li plateau. Such behavior corresponds to that of the generation and filtering of internal gravity waves in Pop II stars. As shown by Talon \& Charbonnel (2004) gravity waves are indeed very efficient in dwarf stars along the plateau up to an effective temperature of $\sim 6300 \mathrm{~K}$. There they dominate the transport of angular momentum and should lead to a quasi-solid rotation state of the stellar radiative zones on very short time-scales. As a result the surface Li depletion is expected to be independant of the initial angular momentum distribution in this range of effective temperatures. This should alleviate the difficulty encountered by the classical rotating models which predict that a range of initial angular momenta generates a range of $\mathrm{Li}$ depletion and that the scatter increases with the average Li depletion. In more massive stars however the efficiency of the gravity waves strongly decreases and internal differential rotation is expected to be maintained under the effect of meridional circulation and turbulence induced by rotation ${ }^{19}$. Consequently variations of the original angular momentum from star to star would lead to more Li dispersion and to more frequent abnormalities in the case of the most massive stars where gravity waves are not fully efficient. In other words internal gravity waves are expected to dominate completely the transport of angular momentum and should lead to higher Li homogeneity in the less massive, rigid rotators than in the stars lying on or originating from the hot side of the plateau. The proper treatment of the effects of gravity waves together with those of atomic diffusion, meridional circulation and shear turbulence has now to be undertaken in stellar evolution models in order to test the real and interactive consequences of all these complex mechanisms (Talon \& Charbonnel 2005).

\section{Summary and conclusions}

The Spite \& Spite (1982a,b) discovery has set the stage for analyses to follow focusing on the determination of the lithium abundances in the most metal-poor, thus the oldest stars of our Galaxy. In view of the crucial importance of this problem for

\footnotetext{
19 The mass dependance of the gravity waves efficiency leads to a natural explanation of the fast horizontal branch rotators. It also provides a solution to the enigma of the so-called $\mathrm{Li}$ dip observed in Pop I stars in terms of rotational mixing, forming a coherent picture of mixing in main sequence stars of all masses. Also, gravity waves are able to shape the Sun's flat rotation profile deduced from helioseismology. See Talon \& Charbonnel $(2003,2004)$ and Charbonnel \& Talon (2005) for more details.
} 
cosmological, galactic and stellar implications, all the observational and theoretical aspects have been the subject of very lively debates. In the present paper we tackled further the most critical issues by revisiting the $\mathrm{Li}$ data available in the literature. Our sample was assembled following strict selection criteria on the quality of the original analysis, i.e., high resolution and high signal to noise spectra.

In the first part we focused on the systematic uncertainties affecting the determination of $\mathrm{Li}$ abundances. We explored in detail the temperature scale issue and put special emphasis on reddening with the aim of deriving a tool as consistent as possible for all our sample stars. In order to do so, we chose to derive photometric temperatures using Strömgren $u v b y-\beta$ photometry which was the only one available for our entire sample. During these steps, we identified one of the major drawbacks of such determinations, namely an accurate estimate of the reddening affecting each of our stars. We derived four sets of effective temperatures based on different assumptions for the interstellar reddening excess values, $E(b-y)$. We tried to evaluate the effect of using reddening values taken from different sources on the derived temperature scales and in turn on the derived $A(\mathrm{Li})$ abundance, showing that an unpredictable mix of different reddening sources could be held responsible for opposite findings, on the same dataset, about the presence of dispersion and/or slope on the Li plateau. Finally, we selected as our best and final $T_{\text {eff }}$ scale the one ( $\left.T_{\text {eff }}(2)\right)$ that has been derived from de-reddening all the stars, except those with negative $E(b-y)$ values.

In order to keep as many stars as possible in our analysis we had to make some compromises on the derivation of the effective temperature of some objects. This contaminated our complete sample of 118 stars which was finally subdivided as follows: 1) the clean sample which contains 91 stars for which the complete set of Strömgren photometric indices are available and for which the Schuster \& Nissen (1989) calibration for the interstellar reddening excess is applicable; 2$)$ the $\beta$ sample which includes 20 stars for which the reddening $E(b-y)$ value was derived from averaging different sources of $E(B-V)$ via the Crawford's formula; 3 ) the $u b v y$ sample which contains 7 stars for which one of the $u b v y$ photometric indices was found to fall just slightly outside the allowed intervals for the application of the Schuster \& Nissen calibrations. We made several tests to quantify the influence of these compromises on the statistical analysis and on our final assessments. In order to guarantee the absence of spurious differences and conclusions due to the use of different criteria in the determination of the effective temperature, all our results (summarised hereafter) regarding the characteristics of the plateau were given for the clean sample on one hand and for the complete (i.e., clean $+\beta+u b v y$ ) sample on the other hand. This approach should provide as high accuracy and reliability as possible for one of the largest sample yet studied.

We then derived the lithium abundances for the various subsamples using the different sets of temperature. This was done using the arithmetic mean of the equivalent widths and the $1 \sigma$ uncertainty of the $670.7 \mathrm{~nm}$ line as reported in the literature from which we had assembled the sample. The lithium abundance was first derived under LTE assumptions for all the $T_{\text {eff }}$ scales studied here and then NLTE corrections were applied. With these NLTE Li abundances we determined the mean $\mathrm{Li}$ value and dispersion along the plateau for our sample as a whole. In order to avoid any contamination by lithium production from various stellar sources we then restricted our discussion only to those stars with $[\mathrm{Fe} / \mathrm{H}]$ lower than -1.5 . In this metallicity range, we considered as plateau stars those with an effective temperature higher than 5700 or $6000 \mathrm{~K}$. Stars with Li upper limits were excluded from the analysis and are discussed separately later. We found sligthly different results for the mean lithium abundance and for the dispersion depending on the lowest limit on $T_{\text {eff }}$ and on the sample under consideration, i.e. clean or complete. We note however that the pollution due to our compromises on the derivation of the effective temperature of some of our objects has a negligible impact on our conclusions. The effect of having assumed a lower limit on $[\mathrm{Fe} / \mathrm{H}]$ in the colour $-T_{\text {eff }}$ calibration used here to derive our sets of effective temperatures is also negligible. This assumption has only the effect of raising the $A(\mathrm{Li})$ plateau abundances by $0.03 \mathrm{dex}$, on average, making our conclusions quite robust.

In the case of the stars of the clean sample with $T_{\text {eff }} \geq 6000 \mathrm{~K}$ we obtain

$A(\mathrm{Li})_{\mathrm{NLTE}}=2.2243 \pm 0.0748$.

This is a factor of 2.48 to 2.74 lower (depending on the SBBN study we rely on, i.e., Coc et al. 2004; Cyburt 2004; or Serpico et al. 2004) than the prediction for a standard Big Bang corresponding to the WMAP estimate of $\Omega_{\mathrm{b}} h^{2}$. The relatively low lithium abundance seen in metal-poor halo stars is a very robust result. Assuming the correctness of the CMB constraint on the value of the baryon-to-photon ratio we are then left with the conclusion that the $\mathrm{Li}$ abundance seen at the surface of halo stars is not the pristine one, but that these stars have undergone surface lithium depletion at some point during their evolution.

In the second part of the present paper we further pushed the constraints on lithium depletion in halo stars. Using our homogeneized data we looked at the Li plateau by considering the evolutionary status of each star. This could be done using the Hipparcos parallaxes which were available for almost all our initial sample stars. This step of the analysis proved to be crucial since a contamination exists from post-main sequence stars, which has to be removed in order to precisely determine the depletion factor along the plateau. Several conclusions could then be drawn.

Again the mean lithium abundance for the dwarf stars depends on the lowest effective temperature chosen to delimit the plateau and slightly varies when one considers the clean sample only or the complete one. The mean lithium plateau value for the dwarf stars of the clean sample with $T_{\text {eff }} \geq 6000 \mathrm{~K}$ is

$A(\mathrm{Li})_{\mathrm{NLTE}}=2.2154 \pm 0.0737$.

This is a factor of 2.53 to 2.8 lower than the WMAP + SBBN primordial $\mathrm{Li}$ value. Note that for the dwarf stars of the clean sample with $T_{\text {eff }} \geq 5700 \mathrm{~K}$ we derive a mean value of

$A(\mathrm{Li})_{\mathrm{NLTE}}=2.1768 \pm 0.0711$ 
which is 2.76 to 3.06 times lower than the CMB-derived value. We find no evidence of intrinsic Li dispersion along the plateau when only the dwarf stars are considered.

A very surprising result was found for the first time: Whatever the subsample we considered, the mean value of $A(\mathrm{Li})_{\text {NLTE }}$ always appears to be higher (although compatible within the errors) for the subgiant stars than for the dwarfs, except for the most metal-deficient objects (i.e., with $[\mathrm{Fe} / \mathrm{H}] \leq$ -2.5) where the mean lithium abundance is very similar in both evolutionary status. The mean lithium value for the post-main sequence stars of the clean sample with $T_{\mathrm{eff}} \geq 6000 \mathrm{~K}$ is

$A(\mathrm{Li})_{\mathrm{NLTE}}=2.2349 \pm 0.0769$

and with $T_{\text {eff }} \geq 5700 \mathrm{~K}$ is

$A(\mathrm{Li})_{\mathrm{NLTE}}=2.2599 \pm 0.0997$.

Additionally the post-main sequence stars show a nonnegligible Li dispersion. This is true at the turnoff and all along the Hertzsprung gap. This feature recalls that observed in subgiant stars of the M92 globular cluster.

We checked that the above conclusions do not change when we exclude the stars that belong to multiple systems or show variability. We could confirm that binary stars do not exhibit lithium abundances significantly different to their single counterparts.

We finished our close examination of our sample by looking at the stars that present deviant Li abundances, i.e., the stars with strong Li deficiency (the so-called ULDs) and those with an abnormally high Li content. We found that all of them lie on or originate from the hot side of the plateau. This agrees with our finding that the turnoff and subgiant stars present a slightly higher Li mean value and dispersion than the dwarfs. These results indicate that the post-main sequence halo stars experienced a Li history slightly different from that of the less massive plateau dwarfs. We suggest that such a behavior may be the signature of a transport process of the chemicals and of angular momentum whose efficiency changes on the blue edge of the plateau. This in agreement with the fact that most of the ULDs are presently rotating faster than the Li-normal stars. Our analysis provided thus some crucial clues to the internal processes that may be involved in modifying the surface $\mathrm{Li}$ abundances in halo stars. Since internal gravity waves coupled with rotationinduced mixing are expected to lead to higher Li homogeneity with $T_{\text {eff }}$ in the plateau stars than in the more massive stars lying on or originating from the hot side of the plateau, such a model is favoured. Although we excluded the ULDs stars from the analysis and focused on the "normal-Li" stars to derive the Li mean values and the trends with $T_{\text {eff }}$ and metallicity, these objects should not be excluded from hydrodynamical studies of the Li depletion mechanisms that affect the Pop II stars.

Acknowledgements. We dedicate this paper to the two new little lithium boys Angel David and Martin Lou. F.P. is indebted to CNRS and the University Paul Sabatier in Toulouse for the Visiting Researcher contracts awarded. F.P. warmly thanks the Laboratoire d'Astrophysique de Toulouse et Tarbes, France for the warm hospitality received during her extended and frequent visits. C.C. thanks the french Programme National de Physique Stellaire and Programme
National Galaxies for financial support. Part of this work has been carried out at the University of Washington (Seattle, USA) where both F.P. and C.C. participated in the INT Programme on Nucleosynthesis (April 2002). We used the SIMBAD data base operated at the CDS (Strasbourg, France).

\section{References}

Akritas, M. G., \& Bershady, M. A. 1996, ApJ, 470, 706

Alonso, A., Arribas, S., \& Martínez-Roger, C. 1996, A\&A, 313, 873

Alonso, A., Arribas, S., \& Martínez-Roger, C. 1999, A\&AS, 140, 261

Alonso, A., Arribas, S., \& Martínez-Roger, C. 2001, A\&A, 376, 1039 (Erratum)

Ardeberg, A., \& Lidgren, H. 1991, A\&A, 244, 310

Asplund, M., Carlsson, M., \& Botnen, A. V. 2003, A\&A, 399, L31

Asplund, M., Nissen, P. E., Lambert, D. L., Primas, F., \& Smith, V. V. 2005, ApJ, to be submitted

Bahcall, J. N., Pinsonneault, M. H., Basu, S., \& Christensen-Dalsgaard, J. 1997, Phys. Rev. Lett., 78, 171

Bania, T. M., Rood, R. T., \& Balser, D. S. 2002, Nature, 415, 54

Barklem, P. S., Belyaev, A. K., \& Asplund, M. 2003, A\&A, 409, L1

Bennett, C. L., Halpern, M., Hinshaw, G., et al. 2003, ApJS, 148, 1

Boesgaard, A. M., Deliyannis, C. P., Stephens, A., \& King, J. K. 1998, ApJ, 493, 206 (B98)

Boesgaard A. M., King, J. K., Deliyannis, C. P., \& Vogt, S. S. 1999, AJ, 117, 492

Boesgaard, A. M., \& Steigman, G. 1985, ARA\&A, 23, 319

Bonifacio, P. 2002, A\&A, 395, 515

Bonifacio, P., \& Molaro, P. 1997, MNRAS, 285, 847 (BM97)

Bonifacio, P., \& Molaro, P. 1998, ApJ, 500, L175

Bonifacio, P., Pasquini, L., Spite, F., et al. 2002, A\&A, 390, 91 (BSP02)

Brown, L., \& Schramm, D. N. 1988, ApJ, 329, L103

Burstein, D., \& Heiles, C. 1982, AJ, 87, 1165

Carlsson, M., Rutten, R. J., Bruls, J. H. M. J., \& Shcchukina, N. G. 1994, A\&A, 288, 860

Carney, B. W. 1983, AJ, 88, 610 (C83)

Carney, B. W., Latham, D. W., Laird, J. B., \& Aguilar, L. A. 1994, AJ, 107,2240

Carney, B. W., Latham, D. W., Laird, J. B., Grant, C. E., \& Morse, J. A. 2001, AJ, 122, 3419

Carney, B. W., Latham, D. W., Stefanik, R. P., Laird, J. B., Morse, J. A. 2003, AJ, 125, 293

Castelli, F., Gratton, R., \& Kurucz, R. L. 1997, A\&A, 328, 841

Castilho, B.V., Gregorio-Hetem, J., Spite, F., Barbuy, B., \& Spite, M. 2000, A\&A, 364, 674

Cayrel, R., Spite, M., Spite, F., et al. 1999a, A\&A, 343, 923

Cayrel, R., Lebreton, Y., \& Morel, P. 1999b, Ap\&SS, 265, 87

COBE Diffuse Infrared Background Experiment (DIRBE) Explanatory Supplement 1995, ed. M. G. Hauser, et al. (Greenbelt, MD: NASA/GSFC)

Chaboyer, B., \& Demarque, P. 1994, ApJ, 433, 510

Charbonnel, C. 1995, ApJ, 453, L41

Charbonnel, C. 2002, Nature, 415, 27

Charbonnel, C., \& Talon, S. 2005, Science, in press

Coc, A., Vangioni-Flam, E., Descouvemont, P., Adahchour, A., \& Angulo, C. 2004, ApJ, 600, 544

Crawford, D. L. 1975, PASP, 87, 481

Croft, R. Q., Weinberg, D. H., Bolte, M., et al. 2002, ApJ, 581, 20

Cyburt, R. H. 2004, Phys. Rev. D, 70, 023505

Dekker, H., D’Odorico, S., Kaufer, A., Delabre, B., \& Kotzlowski, H. 2000, SPIE, 4008, 534

Deliyannis, C. P., Demarque, P., \& Kawaler, S. D. 1990, ApJS, 73,21 
Deliyannis, C. P., \& Malaney, R. A. 1995, ApJ, 453, 810

Deliyannis, C. P., Pinsonneault, M. H., \& Duncan, D. K. 1993, ApJ, 414,740

Eddington, A. S. 1929, MNRAS, 90, 54

ESA 1997, The Hipparcos and Tycho Catalogues, ESA SP-1200

Ford, A., Jeffries, R. D., Smalley, B., et al. 2002, A\&A, 393, 617

Fulbright, P. 2000, AJ, 120, 1841

Fuhrmann, K., Axer, M., \& Gehren, T. 1994, A\&A, 285, 585

Fuhrmann, K., \& Bernkopf, J. 1999, A\&A, 347, 897

Gnedin, N. Y., \& Hamilton, A. J. 2002, MNRAS, 334, 107

Gutierrez, C. M., García López, R. J., Rebolo, R., Martín, E. L., \& Francois, P. 1999, A\&AS, 137, 93

Hakkila, J., Myers, J. M., Stidham, B. J., \& Hartmann, D. H. 1997, AJ, 114, 2043

Hauck, B., \& Mermilliod, M. 1998, A\&AS, 129, 431

Hobbs, L. M., \& Thorburn, J. A. 1991, ApJ, 375, 116

Hobbs, L. M., \& Thorburn, J. A. 1994, ApJ, 428, L25

Hobbs, L. M., \& Thorburn, J. A. 1997, ApJ, 491, 722

Hobbs, L. M., Thorburn, J. A., \& Welty, D. E. 1991, ApJ, 373, L47

Hobbs, L. M., Thorburn, J. A., \& Rebull, L. M. 1999, ApJ, 523, 797

IRAS Catalogs and Atlases: Explanatory Supplement 1988, ed. Beichman, et al.

Izotov, Y. I., \& Thuan, T. X. 2004, ApJ, 602, 200

Jedamzik, K. 2004, Phys. Rev. D, 70, 063524

King, J. R. 1993, AJ, 106, 1206 (K93)

King, J. R., Deliyannis, C. P., \& Boesgaard, A. M. 1996, AJ, 112, 2839

King, J. R., Stephens, A., Boesgaard, A. M., \& Deliyannis, C. P. 1998, AJ, 115, 666

Kirkman, D., Tytler, D., Suzuki, N., O’Meara, J. M., \& Lubin, D. 2003, ApJS, 149, 1

Knude, J. 1979, A\&A, 77, 198

Kuo, C. L., Ade, P., Bock, J. J., et al. 2004, ApJ, 600, 32

Kurucz, R. L. 1993, CD-ROMS \#1,13,18 (Washington, DC: US Government Printing Office)

Laird, J. B., Carney, B. W., \& Latham, D. W. 1988, AJ, 95, 1843

Lallement, R., Welsh, B. Y., Vergely, J. L., Crifo, F., \& Sfeir, D. 2003, A\&A, 411, 447

Lambert, D. L. 1995, A\&A, 301, 478

Latham, D. W., Stefanik, R. P., Torres, G., et al. 2002, AJ, 124, 1144

Lejeune, T., Cuisinier, F., Buser, R. 1998, A\&AS, 130, 65

Lemoine, M., Schramm, D. N., Truran, J. W., Copi, C. J. 1997, ApJ, 478, 554

Meléndez, J., \& Ramírez, I. 2004, ApJ, 615, L33

Michaud, G., Fontaine, G., \& Beaudet, G. 1984, ApJ, 282, 206

Molaro, P. 1991, MmSAI, 62, 189

Molaro, P., Primas, F., \& Bonifacio, P. 1995, A\&A, 295, L47

Molaro, P., Bonifacio, P., Castelli, F., \& Pasquini, L. 1997, A\&A, 319, 593

Nissen, P. E. 1994, Rev. Mex. Astron. Astrofis., 29, 129

Nissen, P. E., Asplund, M., Hill, V., \& D’Odorico, S. 2000, A\&A, 357, L52

Nissen, P. E., Primas, F., Asplund, M., \& Lambert, D. L. 2002, A\&A, 390, 235

Norris, J. E., Ryan, S. G., \& Stringfellow, G. S. 1994, ApJ, 423, 386

Norris, J. E., Ryan, S. G., Beers, T. C., \& Deliyannis, C. P. 1997, ApJ, 485,370

Olive, K. A., \& Skillman, E. D. 2004, ApJ, 617, 290

Olive, K. A., Steigman, G., \& Walker, T. P. 2000, Phys. Rep., 333, 389

Parizot, E., \& Drury, L. 1999, A\&A, 349, 673

Pasquini, L., \& Molaro, P. 1996, A\&A, 307, 761

Pearson, T. J., Mason, B. S., Readhead, A. C. S., et al. 2003, ApJ, 591, 556

Percival, W. J., Baugh, C. M., \& Bland-Hawthorn, J. 2001, MNRAS, 327,1297
Pilachowski, C. A., Sneden, C., \& Booth, J. 1993, ApJ, 407, 699 (PSB93)

Pinsonneault, M. H., Deliyannis, C. P., \& Demarque, P. 1991, ApJ, 967, 239

Pinsonneault, M. H., Walker, T. P., Steigman, G., \& Narayanan, V. K. 1999, ApJ, 527, 180

Pinsonneault, M. H., Charbonnel, C., \& Deliyannis, C. P. 2000, IAU Symp., 198, p.74

Pinsonneault, M. H., Steigman, G., Walker, T. P., \& Narayanan, V. K. 2002, ApJ, 574, 398

Prantzos, N. 2004, ESO Astrophysics Symposium on Chemical abundances and mixing in stars in the Milky Way and its satellites (ed. L. Pasquini, \& S. Randich) [arXiv: astro-ph/0411569]

Press, W. H., Teukolski, A. A., Vetterling, W. P., \& Flannery, B. P. 1992, Numerical Recipes, 2nd edn. (Cambridge: Cambridge Univ. Press)

Primas, F., Duncan, D. K., Peterson, R. C., \& Thorburn, J. A. 1999, A\&A, 343, 545

Proffitt, C. R., \& Michaud, G. 1991, ApJ, 371, 584

Ramírez, I., \& Meléndez, J. 2005a, ApJ, in press [arXiv:astro-ph/0503108] (RM05a)

Ramírez, I., \& Meléndez, J. 2005b, ApJ, in press [arXiv: astro-ph/0503110] (RM05b)

Reeves, H., Audouze, J., Fowler, W. A., \& Schramm, D. N. 1973, ApJ, 179, 909

Richard, O., Vauclair, S., Charbonnel, C., \& Dziembowski, W. A. 1996, A\&A, 312, 1000

Richard, O., Michaud, G., Richer, J., et al. 2002, ApJ, 568, 979 (Ri02)

Richard, O., Michaud, G., \& Richer, J. 2005, ApJ, 619, 538

Romano, D., Matteucci, F., Molaro, P., \& Bonifacio, P. 1999, A\&A, 352, 117

Romano, D., Tosi, M., Matteucci, F., \& Chiappini, C. 2003, MNRAS, 346, 295

Ryan, S. G., \& Deliyannis, C. P. 1998, ApJ, 500, 398

Ryan, S. G., \& Elliott, L. 2004, Proc. ESO/Arcetri Conf. Chem. Abundances and Mixing in Stars in the Milky Way and its Satellites, ed. L. Pasquini, \& S. Randich [arXiv:astro-ph/0410472]

Ryan, S. G., Beers T. C., Deliyannis, C. P., \& Thorburn, J. A. 1996, ApJ, 458, 543 (R96)

Ryan, S. G., Norris, J. E., \& Beers, T. C. 1998, ApJ, 506, 892

Ryan, S. G., Norris, J. E., \& Beers, T. C. 1999, AJ, 523, 654 (R99)

Ryan, S. G., Beers, T. C., Kajino, T., \& Rosolankova, K. 2001a, ApJ, 547, 231

Ryan, S. G., Kajino, T., Beers, T. C., et al. 2001b, ApJ, 549, 55

Ryan, S. G., Gregory, S. G., Kolb, U., Beers, T. C., \& Kajino, T. 2002, ApJ, 571, 501 (R02)

Salaris, M., \& Weiss, A. 2002, A\&A, 388, 492, SW02

Schlegel, D. J., Finkbeiner, D. P., \& Davis, M. 1998, ApJ, 500, 525

Serpico, P. D., Esposito, S., Iocco, F., et al. 2004, JCAP, 12, 10S

Schuster, W. J., \& Nissen, P. E. 1988, A\&AS, 73, 225

Schuster, W. J., \& Nissen, P. E. 1989, A\&A, 221, 65 (Paper II)

Smith, V. V., Lambert, D. L., \& Nissen, P. E. 1993, ApJS, 408, 262

Smith, V. V., Lambert, D. L., \& Nissen, P. E. 1998, ApJ, 506, 405

Smith, V. V., Vargas-Ferro, O., Lambert, D. L., \& Olgin, J. G. 2001, ApJ, 121, 453

Spergel, D. N., Verde, L., Peiris, H. V., et al. 2003, ApJS, 148, 175

Spite, M., \& Spite, F. 1982a, Nature, 297, 483

Spite, F., \& Spite, M. 1982b, A\&A, 115, 357

Spite, M., Francois, P., Nissen, P. E., \& Spite, F. 1996, A\&A, 307, 172

Suzuki, T. K., \& Inoue, S. 2004, Publ. Astron. Soc. Austr., 21, 148

Suzuki, T. K., Yoshii, Y., \& Kajino, T. 1999, ApJ, 522, L125

Suzuki, T. K., Yoshii, Y., \& Beers, T. C. 2000, ApJ, 540, 99 
Talon, S., \& Charbonnel, C. 2003, A\&A, 405, 1025

Talon, S., \& Charbonnel, C. 2004, A\&A, 418, 1051

Talon, S., \& Charbonnel, C. 2005, A\&A, 440, 981

Thorburn, J. A. 1992, ApJ, 399, L83

Thorburn, J. A. 1994, ApJ, 421, 318

Théado, S., \& Vauclair, S. 2001, A\&A, 375, 70

Thévenin, F., Charbonnel, C., de Freitas Pacheco, J. A., et al. 2001, A\&A, 373, 905

Tosi, M. 1998, Space Science Rev., 84, 207

Travaglio, C., Randich, S., Galli, D., et al. 2001, A\&A, 559, 909 van Altena, W. F., Lee, J. T., \& Hoffleit, D. 1989, BAAS, 21, 1135

VandenBerg, D. A., Richard, O., Michaud, G., \& Richer, J. 2002, ApJ, 571,487

Vangioni-Flam, E., Cassé, M., Cayrel, R., et al. 1999, New Astron., 4, 245

Vauclair, S. 1988, ApJ, 335, 971

Vauclair, S., \& Charbonnel, C. 1995, A\&A, 295, 715

Vauclair, S., \& Charbonnel, C. 1998, ApJ, 502, 372

Walker, T. P., Viola, V. E., \& Mathews, G. J. 1985, ApJ, 299, 745

Weiss, A., \& Charbonnel, C. 2004, MmSAI, 75, 347 


\section{Online Material}


Table 3. The data sample and its main characteristics, as found in the literature.

\begin{tabular}{|c|c|c|c|c|c|c|c|c|c|}
\hline HIP & $\mathrm{HD}$ & $\mathrm{BD} / \mathrm{CD}$ & $G$ & $\begin{array}{r}V \\
\text { mag }\end{array}$ & $\begin{array}{c}T_{\text {eff Lit }} \\
\quad \mathrm{K}\end{array}$ & $\begin{array}{l}\log g_{\text {Lit }} \\
\mathrm{cm} \mathrm{s}^{-1}\end{array}$ & $\begin{array}{c}{[\mathrm{Fe} / \mathrm{H}]_{\mathrm{Lit}}} \\
\quad \operatorname{dex}\end{array}$ & $\begin{array}{c}E W_{\mathrm{Lit}} \\
\mathrm{m} \AA\end{array}$ & $\operatorname{Ref}^{a}$ \\
\hline 484 & 97 & -206718 & & 9.660 & 5000 & & -1.23 & 12.1 & 5 \\
\hline 911 & & & $266-060$ & 11.800 & 5890 & 2.2 & -1.84 & 30.8 & 3 \\
\hline 2413 & 2665 & +5670 & & 7.729 & $5050 \ldots 5100$ & 3.6 & $-1.80 \ldots-1.89$ & $15.0 \ldots 20.0$ & 5,12 \\
\hline 3026 & 3567 & $-09122 \mathrm{~B}$ & $270-023$ & 9.252 & $5858 \ldots 5930$ & 3.7 & $-1.20 \ldots-1.34$ & 45.0 & 1,4 \\
\hline 3430 & & +7131 & $242-065$ & 10.202 & $6026 \ldots 6170$ & & $-1.91 \ldots-2.20$ & 31.0 & 1,4 \\
\hline 3446 & & & $268-032$ & 12.105 & 5910 & & -3.50 & 27.0 & 1 \\
\hline 3554 & 4306 & -10155 & & 9.017 & 4950 & & -2.87 & 17.7 & 5 \\
\hline 3564 & & & $033-009$ & 10.598 & 5500 & 3.0 & -1.27 & 35.2 & 5 \\
\hline 4343 & 5426 & $-34347 *$ & & 9.629 & 5100 & & -2.08 & 9.1 & 5 \\
\hline 5445 & 6755 & +60170 & $244-016$ & 7.717 & 5200 & & $-1.39 \ldots-1.52$ & 15.3 & 5 \\
\hline 8314 & & +7294 & $245-032$ & 9.936 & $6160 \ldots 6460$ & 3.0 & $-1.60 \ldots-1.80$ & 27.0 & $1,3,5$ \\
\hline 8572 & & -10388 & $271-162$ & 10.342 & $5950 \ldots 6040$ & 4.0 & -2.51 & 27.0 & 1,5 \\
\hline 10140 & & +29366 & $074-005$ & 8.768 & $5425 \ldots 5760$ & $3.8 \ldots 4.1$ & -1.00 & 14.0 & 8 \\
\hline 11952 & 16031 & -13482 & & 9.771 & $6020 \ldots 6114$ & $3.9 \ldots 4.2$ & $-1.60 \ldots-2.20$ & 28.0 & $1,3,4,12$ \\
\hline 12529 & & +09352 & 076-021 & 10.172 & $5931 \ldots 6080$ & 4.0 & $-2.15 \ldots-2.30$ & 34.0 & 1,4 \\
\hline 12807 & & & $004-037$ & 11.310 & $6050 \ldots 6337$ & 4.4 & $-2.73 \ldots-3.31$ & $20.0 \ldots 25.9$ & $1,2,4$ \\
\hline 13749 & & $-301121^{*}$ & & 10.436 & 5100 & & -1.62 & 14.6 & 5 \\
\hline 14594 & 19445 & +25495 & $037-026$ & 8.053 & $5825 \ldots 6050$ & $4.2 \ldots 4.45$ & $-2.00 \ldots-2.15$ & $34.0 \ldots 41.0$ & $1,4,12$ \\
\hline 15481 & & $-331173^{*}$ & & 10.920 & $6250 \ldots 6260$ & 4.5 & $-3.14 \ldots-3.30$ & $12.0 \ldots 17.2$ & $1,2,9$ \\
\hline 16072 & & & $005-036$ & 10.806 & 5600 & & -1.35 & 44.2 & 5 \\
\hline 16214 & 21581 & -00552 & & 8.708 & 4900 & 3.8 & -1.73 & 17.3 & 5 \\
\hline 16404 & & +66268 & $246-038$ & 9.912 & 5279 & 4.5 & -2.94 & 10.0 & 4 \\
\hline 17001 & & $-241782^{*}$ & & 9.920 & 5300 & & -2.70 & 12.6 & 5 \\
\hline 18082 & 24289 & -04680 & 080-028 & 9.969 & $5570 \ldots 5866$ & 3.7 & $-1.64 \ldots-2.22$ & $45.0 \ldots 51.0$ & $1,4,5,10,11,13$ \\
\hline 18802 & 25704 & $-57806^{*}$ & & 8.112 & 5625 & & -1.06 & 23.0 & 10 \\
\hline 19797 & 284248 & +21607 & $008-016$ & 9.227 & $5960 \ldots 6034$ & $4.05 \ldots 4.2$ & $-1.50 \ldots-1.60$ & $25.0 \ldots 31.6$ & $1,4,12,13$ \\
\hline 21609 & 29907 & -65253 & & 9.850 & $5200 \ldots 5350$ & 3.8 & -1.60 & $16.0 \ldots 26.0$ & 10,12 \\
\hline 22632 & 31128 & $-271935^{*}$ & & 9.131 & 5825 & 4.3 & -1.40 & 35.0 & 12 \\
\hline 23344 & & +03740 & 084-029 & 9.810 & $6075 \ldots 6340$ & 3.7 & $-2.01 \ldots-2.90$ & $19.5 \ldots 21.0$ & $1,2,4,5,12$ \\
\hline 24316 & 34328 & $-591024^{*}$ & & 9.430 & $5725 \ldots 5830$ & $4.4 \ldots 4.6$ & $-1.50 \ldots-1.73$ & $29.0 \ldots 36.0$ & $9,10,12$ \\
\hline 25659 & & $-292277^{*}$ & & 11.610 & 5660 & & -2.00 & 43.0 & 1 \\
\hline 28887 & 251611 & & $102-047$ & 10.313 & 5200 & & $-1.64 \ldots-1.69$ & 18.0 & 5,10 \\
\hline 29759 & & +371458 & $098-058$ & 8.916 & $5200 \ldots 5510$ & $3.0 \ldots 3.8$ & $-1.86 \ldots-2.39$ & $11.0 \ldots 16.0$ & $4,5,7,12$ \\
\hline 29992 & 44007 & -141399 & & 8.053 & 4900 & & -1.50 & 18.7 & 5 \\
\hline 30098 & & +381456 & $101-034$ & 10.800 & & & -1.98 & 16.0 & 11 \\
\hline 30668 & 45282 & +031247 & & 8.027 & 5400 & 3.9 & $-1.29 \ldots-1.51$ & $9.9 \ldots 16.0$ & 5,11 \\
\hline 32567 & & & $192-043$ & 10.321 & 6130 & & $-1.43 \ldots-1.49$ & $34.5 \ldots 73.0$ & 3,11 \\
\hline 34630 & & & $088-010$ & 11.855 & $5856 \ldots 6010$ & 3.85 & $-2.20 \ldots-2.70$ & 30.0 & 1,4 \\
\hline 36174 & & +191730 & $088-027$ & 10.740 & 6058 & 3.8 & $-1.47 \ldots-1.65$ & $35.8 \ldots 45.0$ & 3,11 \\
\hline 36430 & & & 090-003 & 10.414 & $5786 \ldots 5900$ & $3.0 \ldots 3.55$ & $-1.93 \ldots-2.47$ & $43.5 \ldots 45.0$ & $1,4,5,7,11$ \\
\hline 36513 & & +241676 & $088-032$ & 10.800 & $6170 \ldots 6300$ & 3.78 & $-2.59 \ldots-2.71$ & $19.0 \ldots 27.0$ & $1,2,4,6,7,11$ \\
\hline 37671 & & $+002058 \mathrm{~A}$ & $112-043$ & 10.208 & 6034 & & -1.48 & 37.6 & 3 \\
\hline 38541 & 64090 & +311684 & $090-025$ & 8.309 & $5294 \ldots 5441$ & $4.5 \ldots 4.7$ & $-1.70 \ldots-1.82$ & $11.0 \ldots 13.0$ & $4,7,12,13$ \\
\hline 40778 & 233511 & +541216 & $194-022$ & 9.728 & 5900 & 4.2 & -1.60 & 31.0 & 12 \\
\hline 42592 & 74000 & -152546 & $043-043$ & 9.674 & $6040 \ldots 6224$ & 4.08 & $-1.80 \ldots-2.02$ & $22.1 \ldots 25.0$ & $1,2,4,13$ \\
\hline 42887 & & +251981 & $009-016$ & 9.316 & 6780 & & -1.30 & $<3.7$ & 3 \\
\hline 44605 & & & $115-049$ & 11.603 & $5710 \ldots 5950$ & 5.0 & $-1.86 \ldots-2.20$ & 38.0 & 1,4 \\
\hline 44716 & 78050 & +211969 & 009-047 & 7.679 & $4800 \ldots 4835$ & & $-1.02 \ldots-1.93$ & $<3.0 \ldots<4.0$ & 7,10 \\
\hline 46516 & & +092190 & $041-041$ & 11.147 & $6260 \ldots 6333$ & 3.83 & $-2.80 \ldots-2.90$ & $18.0 \ldots 22.0$ & $1,4,7,11$ \\
\hline 47480 & 83769 & $+012341 p$ & $048-029$ & 10.176 & $6260 \ldots 6313$ & 3.9 & $-2.70 \ldots-2.82$ & $16.7 \ldots 25.0$ & $1,2,4,9,13$ \\
\hline 48152 & 84937 & +142151 & $043-003$ & 8.324 & $6090 \ldots 6375$ & $3.9 \ldots 4.1$ & $-2.00 \ldots-2.49$ & $24.0 \ldots 26.7$ & $1,2,4,5,9,12,13$ \\
\hline
\end{tabular}


Table 3. continued.

\begin{tabular}{|c|c|c|c|c|c|c|c|c|c|}
\hline HIP & HD & $\mathrm{BD} / \mathrm{CD}$ & $G$ & $\begin{array}{r}V \\
\text { mag }\end{array}$ & $\begin{array}{c}T_{\text {eff Lit }} \\
\mathrm{K}\end{array}$ & $\begin{array}{l}\log g_{\text {Lit }} \\
\mathrm{cm} \mathrm{s}^{-1}\end{array}$ & $\begin{array}{c}{[\mathrm{Fe} / \mathrm{H}]_{\text {Lit }}} \\
\quad \operatorname{dex}\end{array}$ & $\begin{array}{c}E W_{\text {Lit }} \\
\mathrm{m} \AA\end{array}$ & Ref. $^{a}$ \\
\hline 48444 & 237846 & +581218 & & 9.960 & 5000 & & -2.66 & & 5 \\
\hline 49371 & 87140 & +551362 & & 8.995 & 5000 & & -1.75 & 24.5 & 5 \\
\hline 49616 & 89499 & $-84102 *$ & & 8.660 & 4875 & & -2.23 & 45.0 & 10 \\
\hline 52771 & & $+292091 b$ & $119-032$ & 10.260 & $5720 \ldots 5815$ & 4.8 & $-1.80 \ldots-1.86$ & 45.0 & 1,4 \\
\hline 53070 & 94028 & +212247 & $058-025$ & 8.222 & $5800 \ldots 6001$ & $3.0 \ldots 4.15$ & $-1.50 \ldots-1.70$ & $35.0 \ldots 36.6$ & $1,4,5,13$ \\
\hline 54639 & & & 010-004 & 11.385 & 5000 & & -2.49 & $<5.0$ & 10 \\
\hline 55022 & 97916 & +022406 & & 9.208 & $6000 \ldots 6124$ & & $-1.10 \ldots-1.38$ & $<3.0$ & 3,5 \\
\hline 55852 & & +042466 & & 10.532 & 5000 & & -2.15 & $<6.0$ & 5 \\
\hline 57244 & & & $122-043$ & 12.020 & 5600 & & -2.80 & 34.0 & 1 \\
\hline 57983 & & -012582 & & 9.610 & 5100 & & -2.29 & 10.2 & 5 \\
\hline 58357 & 103912 & +492098 & $122-057$ & 8.390 & 4744 & & -1.71 & $<4.0$ & 7 \\
\hline 59109 & & -043208 & 013-009 & 9.999 & $6120 \ldots 6200$ & & -2.40 & $25.0 \ldots 25.6$ & 1,9 \\
\hline 59376 & & & $011-044$ & 11.093 & $5930 \ldots 5943$ & 4.08 & $-1.80 \ldots-2.40$ & 29.0 & 1,4 \\
\hline 60387 & 107752 & +122458 & & 10.010 & 4700 & & -2.88 & $<4.0$ & 5 \\
\hline 60632 & 108177 & +022538 & $013-035$ & 9.663 & $5990 \ldots 6200$ & $4.0 \ldots 4.3$ & $-1.50 \ldots-1.98$ & $29.8 \ldots 33.0$ & $1,4,12,13$ \\
\hline 60719 & 108317 & +062613 & & 8.038 & 5300 & & -2.32 & $<4.0$ & 5 \\
\hline 61361 & & & $059-024$ & 12.009 & $5970 \ldots 6108$ & 4.28 & $-2.60 \ldots-2.69$ & 36.0 & 1,2 \\
\hline 61545 & & +282137 & $059-027$ & 10.889 & $6050 \ldots 6107$ & 3.85 & $-2.20 \ldots-2.34$ & $27.0 \ldots 28.0$ & $1,4,7,11$ \\
\hline 61802 & & +132567 & $060-026$ & 9.806 & 5400 & & -1.22 & 13.6 & 5 \\
\hline 62747 & 111721 & -123709 & & 7.972 & 5000 & & -1.34 & 17.2 & 5 \\
\hline 63385 & & +102495 & & 9.723 & 5000 & & -1.83 & 21.3 & 5 \\
\hline 63559 & 113083 & $-269470^{*}$ & & 8.043 & 5750 & & -0.93 & 37.0 & 10 \\
\hline 64786 & 115444 & +372388 & & 8.967 & 4800 & & -2.72 & $<3.0$ & 5 \\
\hline 65201 & 116064 & $-388457 *$ & & 8.797 & $5770 \ldots 5880$ & & $-2.01 \ldots-2.40$ & $26.0 \ldots 30.0$ & $1,9,10$ \\
\hline 65206 & & & $255-032$ & 11.660 & 5760 & & -2.60 & 30.0 & 1 \\
\hline 66354 & & +012831 & $062-052$ & 10.890 & 5150 & & -1.43 & 8.0 & 10 \\
\hline 66673 & & & $064-012$ & 11.468 & $6110 \ldots 6468$ & 4.1 & $-3.17 \ldots-3.50$ & $21.2 \ldots 28.0$ & $1,2,4,7,9,11$ \\
\hline 67655 & 120559 & $-565169^{*}$ & & 7.975 & 5325 & & -1.03 & $<6.0$ & 10 \\
\hline 67863 & 121004 & $-458786^{*}$ & & 9.034 & 5525 & & -0.88 & $<5.0$ & 10 \\
\hline 68321 & & $+342476 b$ & $165-039$ & 10.050 & $6220 \ldots 6282$ & 3.88 & $-2.30 \ldots-2.41$ & 23.0 & 1,4 \\
\hline 68464 & 122196 & $-379083^{*}$ & & 8.729 & 5850 & & -1.93 & 40.0 & 10 \\
\hline 68592 & & & $064-037$ & 11.134 & $6150 \ldots 6432$ & 4.15 & $-2.51 \ldots-3.38$ & $14.0 \ldots 18.2$ & $1,2,4,7,9,11$ \\
\hline 70647 & 126587 & -213903 & & 9.113 & 4750 & & -3.06 & 14.5 & 5 \\
\hline 71087 & & +182890 & & 9.840 & 5000 & & -1.61 & $<0.3$ & 5 \\
\hline 71458 & 128279 & $-2810826^{*}$ & & 8.022 & 5100 & & -2.32 & 11.4 & 5 \\
\hline 72461 & & +262606 & $166-045$ & 9.731 & $5875 \ldots 6053$ & & $-2.30 \ldots-2.70$ & $29.0 \ldots 36.0$ & $1,4,5,7,11,12$ \\
\hline 72561 & & & $066-030$ & 11.049 & 6269 & & -1.66 & $<6.3$ & 3,9 \\
\hline 72920 & & +262621 & $166-054$ & 11.000 & $6090 \ldots 6150$ & & $-2.80 \ldots-2.88$ & $20.0 \ldots 22.5$ & 1,2 \\
\hline 73385 & 132475 & $-214009 v$ & & 8.547 & $5575 \ldots 5788$ & $3.6 \ldots 3.65$ & $-1.50 \ldots-1.93$ & $50.0 \ldots 58.0$ & $4,7,12$ \\
\hline 74079 & 134169 & +042969 & & 7.691 & 5800 & 3.0 & -1.60 & 44.0 & 5 \\
\hline 74234 & 134440 & $-154041 v$ & & 9.445 & 4750 & & $-1.44 \ldots-1.52$ & $<0.9 \ldots<6.0$ & 10,11 \\
\hline 74235 & 134439 & -154042 & & 9.066 & & & -1.92 & $<10.0$ & 11 \\
\hline 76059 & & & $152-035$ & 12.130 & $5491 \ldots 5700$ & $3.8 \ldots 5.1$ & $-1.61 \ldots-1.75$ & 53.7 & 4,9 \\
\hline 76976 & 140283 & -104149 & & 7.211 & $5650 \ldots 5742$ & 3.4 & $-2.37 \ldots-2.60$ & $46.0 \ldots 48.1$ & $2,4,5,7,9,12,13$ \\
\hline 78640 & & +422667 & $180-024$ & 9.859 & $5880 \ldots 6059$ & 4.1 & $-1.50 \ldots-1.67$ & 28.0 & 1,4 \\
\hline 79537 & 145417 & $-576303^{*}$ & & 7.531 & $4850 \ldots 4990$ & & $-1.38 \ldots-2.44$ & $<4.0 \ldots<5.0$ & 7,10 \\
\hline 80679 & & & $180-058$ & 11.304 & & & -2.36 & $<10.0$ & 11 \\
\hline 81170 & 149414 & -033968 & $153-067$ & 9.597 & & & -1.54 & 10.0 & 11 \\
\hline 81276 & & & $202-065$ & 11.220 & 6390 & & -1.50 & $<6.0$ & 3 \\
\hline 83320 & & & $139-008$ & 11.460 & 6025 & & -2.56 & $<5.0$ & 3,7 \\
\hline 85855 & & +233130 & $170-047$ & 8.935 & $5000 \ldots 5250$ & 2.2 & $-2.42 \ldots-2.91$ & $12.0 \ldots 13.0$ & $5,7,12$ \\
\hline 86443 & & +023375 & 020-008 & 9.940 & $5700 \ldots 5891$ & 3.83 & $-2.26 \ldots-2.60$ & $32.0 \ldots 33.0$ & $1,4,5,9$ \\
\hline 86694 & 160617 & -401175 & & 8.723 & $5800 \ldots 5900$ & 3.0 & $-1.60 \ldots-1.91$ & $38.1 \ldots 43.0$ & $2,5,9$ \\
\hline 87062 & & -084501 & $020-015$ & 10.602 & $5600 \ldots 5900$ & $3.0 \ldots 4.0$ & $-1.40 \ldots-2.79$ & $28.3 \ldots 35.1$ & $4,5,9,11$ \\
\hline 87101 & 161770 & -094604 & $154-021$ & 9.682 & 5050 & & -1.38 & $42.0 \ldots 44.9$ & 5,11 \\
\hline
\end{tabular}


Table 3. continued.

\begin{tabular}{|c|c|c|c|c|c|c|c|c|c|}
\hline HIP & HD & $\mathrm{BD} / \mathrm{CD}$ & $G$ & $\begin{array}{r}V \\
\text { mag }\end{array}$ & $\begin{array}{c}T_{\text {eff Lit }} \\
\mathrm{K}\end{array}$ & $\begin{array}{l}\log g_{\text {Lit }} \\
\mathrm{cm} \mathrm{s}^{-1}\end{array}$ & $\begin{array}{c}{[\mathrm{Fe} / \mathrm{H}]_{\mathrm{Lit}}} \\
\quad \operatorname{dex}\end{array}$ & $\begin{array}{c}E W_{\text {Lit }} \\
\mathrm{m} \AA\end{array}$ & Ref. $^{a}$ \\
\hline 87467 & & +362964 & $182-031$ & 10.600 & 6050 & & $-2.53 \ldots-2.60$ & $25.0 \ldots 26.0$ & 1,11 \\
\hline 87693 & & +203603 & $183-011$ & 9.770 & $6000 \ldots 6441$ & $4.0 \ldots 4.3$ & $-2.00 \ldots-2.30$ & $27.0 \ldots 28.0$ & $1,4,5,12$ \\
\hline 88010 & 163810 & -134807 & $154-036$ & 9.633 & $5200 \ldots 5400$ & 4.0 & $-1.23 \ldots-1.55$ & $22.0 \ldots 28.0$ & $5,11,12$ \\
\hline 88827 & & +013597 & $020-024$ & 11.093 & $6150 \ldots 6464$ & & $-0.91 \ldots-2.10$ & $20.0 \ldots 24.5$ & $1,4,9$ \\
\hline 89554 & 166913 & $-596824^{*}$ & & 8.213 & 5955 & 3.3 & -1.60 & 40.0 & 8 \\
\hline 90957 & & +133683 & $141-019$ & 10.571 & $5138 \ldots 5493$ & 3.2 & $-2.39 \ldots-2.91$ & $31.8 \ldots 34.0$ & $4,5,7,11$ \\
\hline 91129 & & & $206-034$ & 11.390 & $6180 \ldots 6191$ & 4.18 & $-2.88 \ldots-3.10$ & $27.0 \ldots 28.0$ & 1,11 \\
\hline 92167 & 175305 & +74792 & $259-035$ & 7.181 & 5190 & & -1.50 & 13.7 & 5 \\
\hline 92775 & & +423187 & $205-042$ & 10.100 & $5251 \ldots 5300$ & 3.7 & $-1.51 \ldots-2.18$ & $15.0 \ldots 24.0$ & $5,7,11$ \\
\hline 95333 & 181743 & $-451317 *$ & & 9.669 & $5790 \ldots 5900$ & $3.5 \ldots 4.5$ & -1.70 & 40.6 & 9 \\
\hline 96115 & 338529 & +263578 & & 9.364 & $6000 \ldots 6310$ & $3.6 \ldots 3.88$ & $-2.30 \ldots-2.58$ & $23.0 \ldots 24.6$ & $1,2,4,5,12$ \\
\hline 98020 & 188510 & +104091 & $143-017$ & 8.830 & $5325 \ldots 5564$ & $4.6 \ldots 4.88$ & $-1.60 \ldots-1.80$ & $18.0 \ldots 20.4$ & $4,12,13$ \\
\hline 98532 & 189558 & -125613 & & 7.728 & $5600 \ldots 5663$ & 3.68 & -1.30 & 42.0 & 4,5 \\
\hline 98974 & 190287 & $-3513902 *$ & & 8.532 & 5100 & & -1.33 & 15.0 & 5 \\
\hline 98989 & & & $024-003$ & 10.463 & $5800 \ldots 5960$ & 3.88 & $-1.60 \ldots-1.81$ & $28.5 \ldots 32.0$ & $1,4,5,11$ \\
\hline 99267 & & +423607 & $125-064$ & 10.110 & 5850 & & $-2.10 \ldots-2.13$ & $43.0 \ldots 47.0$ & 1,11 \\
\hline 99423 & 345957 & +233912 & & 8.889 & $5600 \ldots 5788$ & $3.0 \ldots 3.73$ & $-1.45 \ldots-1.59$ & $77.6 \ldots 80.8$ & 4,5 \\
\hline 100568 & 193901 & -215703 & & 8.647 & $5710 \ldots 5750$ & 4.40 & $-1.13 \ldots-1.20$ & $26.5 \ldots 30.0$ & $1,4,13$ \\
\hline 100682 & 340279 & & $186-026$ & 10.828 & $6180 \ldots 6241$ & & -2.83 & $<2.0$ & $2,3,5$ \\
\hline 100792 & 194598 & +094529 & $024-015$ & 8.345 & $5900 \ldots 6017$ & 4.15 & $-1.37 \ldots-1.60$ & $28.0 \ldots 30.3$ & $1,4,13$ \\
\hline 102337 & & $-3018140 *$ & & 9.936 & 6270 & & -2.10 & $28.8 \ldots 32.4$ & 3,9 \\
\hline 102718 & & +044551 & & 9.710 & $5780 \ldots 5819$ & 3.78 & -1.80 & 29.0 & 1,4 \\
\hline 103269 & & +413931 & $212-007$ & 10.280 & 5300 & 4.6 & -1.70 & 16.0 & 12 \\
\hline 103337 & & -145890 & & 10.210 & 4950 & & -2.07 & 25.8 & 5 \\
\hline 104191 & 200654 & $-5013237^{*}$ & & 9.087 & $5090 \ldots 5203$ & & $-2.91 \ldots-3.16$ & $8.0 \ldots .0 .0$ & 7,9 \\
\hline 104660 & 201889 & +234264 & & 8.056 & $5635 \ldots 5645$ & $3.8 \ldots 4.1$ & -1.10 & $<4.0 \ldots 5.0$ & 4,8 \\
\hline 104658 & 201891 & +174519 & & 8.500 & $5797 \ldots 5909$ & $4.1 \ldots 4.4$ & $-1.22 \ldots-1.40$ & $24.0 \ldots 26.3$ & $1,4,8,13$ \\
\hline 106468 & & $-3514849 *$ & & 10.561 & 6060 & & -2.63 & $28.8 \ldots \ldots 30.8$ & 2,9 \\
\hline 108200 & & & $188-030$ & 11.072 & & & -1.86 & 11.0 & 11 \\
\hline 109558 & & +174708 & $126-062$ & 9.457 & $5941 \ldots 6025$ & $3.58 \ldots 4.0$ & $-1.50 \ldots-2.00$ & $25.0 \ldots 30.0$ & $1,3,4,12$ \\
\hline 110140 & & +074841 & 018-039 & 10.383 & $5976 \ldots 6030$ & 4.0 & $-1.20 \ldots-1.34$ & 37.0 & 1,4 \\
\hline 111195 & & & $018-054$ & 10.706 & $5762 \ldots 5912$ & 3.75 & $-1.30 \ldots-1.52$ & 35.0 & $1,4,5$ \\
\hline 111372 & 213657 & $-4215968 *$ & & 9.654 & 5860 & 3.8 & -2.08 & 31.0 & 9 \\
\hline 114271 & 218502 & -156355 & & 8.254 & $6000 \ldots 6200$ & $3.0 \ldots 4.1$ & $-1.70 \ldots-2.00$ & $27.0 \ldots 30.0$ & $1,5,12$ \\
\hline 114502 & 218857 & -176692 & & 8.943 & 5100 & & -1.87 & 12.1 & 5 \\
\hline 114661 & & +384955 & $190-015$ & 11.021 & $5115 \ldots 5125$ & 5.0 & $-2.69 \ldots-3.00$ & $4.8 \ldots 8.0$ & 4,10 \\
\hline 114962 & 219617 & -146437 & $273-001$ & 8.160 & $5825 \ldots 6012$ & 4.3 & $-1.40 \ldots-1.63$ & $38.0 \ldots 40.0$ & $1,4,12,13$ \\
\hline 115167 & & +024651 & $029-023$ & 10.213 & $5990 \ldots 6102$ & 3.8 & $-1.50 \ldots-2.30$ & $27.0 \ldots 34.0$ & $1,4,11,12$ \\
\hline 115704 & & +592723 & $217-008$ & 10.488 & $6050 \ldots 6134$ & 4.25 & $-1.91 \ldots-2.05$ & 29.0 & $1,4,5$ \\
\hline 117522 & & & $029-071$ & 11.341 & 5640 & & -2.40 & 28.0 & 1 \\
\hline
\end{tabular}

${ }^{a}$ The references are those given in Table $2 . *$ Identifies CD stars. 
Table 4. Photometry and reddening excesses.

\begin{tabular}{|c|c|c|c|c|c|c|c|c|c|c|c|c|c|c|}
\hline HIP & $(b-y)$ & $c 1$ & $m 1$ & $\beta$ & Ref. $^{a}$ & $\begin{array}{r}E(b-y) \\
\beta\end{array}$ & $\begin{array}{c}(B-V) \\
\text { Hip }\end{array}$ & $\begin{array}{c}(B-V) \\
\text { Lit }\end{array}$ & $\begin{array}{c}E(B-V) \\
S 98\end{array}$ & $\begin{array}{c}E(b-y) \\
\mathrm{S} 98\end{array}$ & $\begin{array}{c}E(B-V) \\
\text { H97 }\end{array}$ & $\begin{array}{c}E(b-y) \\
\text { H97 }\end{array}$ & $\begin{array}{c}E(B-V) \\
\quad \text { Lit }\end{array}$ & $\begin{array}{c}E(B-V) \\
\mathrm{BH}\end{array}$ \\
\hline 484 & 0.513 & 0.349 & 0.155 & .. & 2 &. & 0.787 & & 0.021 & 0.016 & & & & \\
\hline 911 & 0.341 & 0.278 & 0.067 & 2.582 & 1 & -0.008 & 0.570 & 0.450 & 0.020 & 0.015 & 0.022 & 0.016 & 0.000 & 0.015 \\
\hline 2413 & 0.549 & 0.360 & 0.078 & 2.730 & 2 & 0.167 & 0.792 & 0.793 & 0.395 & & 0.184 & 0.134 & $\ldots$ & $\ldots$ \\
\hline 3026 & 0.332 & 0.334 & 0.087 & 2.598 & 1 & -0.002 & 0.465 & 0.460 & 0.036 & 0.026 & 0.034 & 0.025 & 0.000 & 0.015 \\
\hline 3430 & 0.309 & 0.360 & 0.040 & $\ldots$ & 2 & $\ldots$ & 0.401 & 0.390 & 0.723 & $\ldots$ & 0.034 & 0.025 & 0.000 & $\ldots$ \\
\hline 3446 & 0.350 & 0.198 & 0.057 & 2.597 & 1 & 0.001 & 0.509 & 0.470 & 0.019 & 0.014 & 0.028 & 0.020 & 0.000 & 0.015 \\
\hline 3554 & 0.518 & 0.348 & 0.052 & 2.529 & 1 & 0.057 & 0.750 & 0.736 & 0.036 & 0.027 & 0.032 & 0.023 & 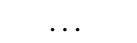 & 0.015 \\
\hline 3564 & 0.411 & 0.283 & 0.102 & 2.573 & 1 & 0.029 & 0.604 & 0.570 & 0.101 & 0.074 & 0.039 & 0.028 & & 0.030 \\
\hline 4343 & 0.498 & 0.326 & 0.080 & $\ldots$ & 2 & $\ldots$ & 0.724 & $\ldots$ & 0.017 & 0.013 & 0.016 & 0.012 & $\ldots$ & $\ldots$ \\
\hline 5445 & 0.486 & 0.281 & 0.118 & 2.541 & 1 & 0.049 & 0.742 & 0.718 & 0.897 & $\ldots$ & 0.082 & 0.060 & $\ldots$ & $\ldots$ \\
\hline 8314 & 0.309 & 0.263 & 0.087 & $\ldots$ & 2 & $\ldots$ & 0.417 & 0.420 & 0.526 & $\ldots$ & 0.050 & 0.036 & 0.040 & $\ldots$ \\
\hline 8572 & 0.326 & 0.359 & 0.049 & 2.602 & 1 & 0.020 & 0.432 & 0.420 & 0.024 & 0.017 & 0.028 & 0.020 & 0.010 & 0.015 \\
\hline 10140 & 0.390 & 0.213 & 0.130 & 2.562 & 1 & -0.013 & 0.580 & 0.570 & 0.099 & 0.073 & 0.019 & 0.014 & 0.000 & 0.060 \\
\hline 11952 & 0.324 & 0.304 & 0.062 & 2.604 & 1 & 0.005 & 0.437 & 0.450 & 0.022 & 0.016 & 0.012 & 0.009 & 0.010 & 0.015 \\
\hline 12529 & 0.345 & 0.245 & 0.054 & 2.609 & 1 & 0.015 & 0.510 & 0.442 & 0.213 & & 0.030 & 0.022 & 0.030 & 0.090 \\
\hline 12807 & 0.363 & 0.307 & 0.031 & 2.570 & 1 & 0.011 & 0.605 & 0.468 & 0.182 & 0.133 & 0.020 & 0.015 & 0.040 & 0.090 \\
\hline 13749 & 0.516 & 0.339 & 0.108 & $\ldots$ & 2 & $\ldots$ & 0.766 & 0.759 & 0.018 & 0.013 & 0.011 & 0.008 & $\ldots$ & 0.015 \\
\hline 14594 & 0.352 & 0.203 & 0.051 & 2.583 & 1 & -0.001 & 0.475 & 0.460 & 0.174 & 0.127 & 0.016 & 0.012 & 0.000 & 0.160 \\
\hline 15481 & 0.292 & 0.356 & 0.011 & 2.618 & 1a & 0.030 & 0.375 & 0.360 & 0.013 & 0.009 & 0.020 & 0.015 & 0.000 & $\ldots$ \\
\hline 16072 & 0.395 & 0.277 & 0.069 & 2.601 & 1 & 0.052 & 0.544 & 0.529 & 0.261 & $\ldots$ & 0.054 & 0.039 & $\ldots$ & 0.120 \\
\hline 16214 & 0.560 & 0.341 & 0.129 & 2.539 & 2 & 0.099 & 0.841 & 0.825 & 0.107 & 0.079 & 0.037 & 0.027 & $\ldots$ & $\ldots$ \\
\hline 16404 & 0.455 & 0.102 & 0.095 & 2.503 & 1 & -0.005 & 0.667 & 0.650 & 1.154 & $\ldots$ & 0.036 & 0.026 & 0.000 & $\ldots$ \\
\hline 17001 & 0.466 & 0.287 & 0.040 & 2.526 & 1 & 0.018 & 0.647 & 0.633 & 0.020 & 0.014 & 0.018 & 0.013 & $\ldots$ & 0.000 \\
\hline 18082 & 0.388 & 0.288 & 0.049 & 2.582 & 1 & 0.040 & 0.532 & 0.517 & 0.214 & $\ldots$ & 0.022 & 0.016 & 0.030 & 0.090 \\
\hline 18802 & 0.371 & 0.275 & 0.118 & 2.570 & 1 & -0.016 & 0.566 & 0.550 & 0.014 & 0.010 & 0.015 & 0.011 & 0.000 & 0.015 \\
\hline 19797 & 0.328 & 0.272 & 0.075 & 2.596 & 1 & -0.010 & 0.442 & 0.450 & 0.479 & $\ldots$ & 0.020 & 0.015 & 0.000 & 0.180 \\
\hline 21609 & 0.452 & 0.132 & 0.106 & 2.538 & 1 & 0.025 & 0.639 & 0.637 & 0.047 & 0.034 & 0.011 & 0.008 & 0.020 & 0.000 \\
\hline 22632 & 0.353 & 0.244 & 0.068 & 2.589 & 2 & 0.004 & 0.497 & 0.490 & 0.037 & 0.027 & 0.018 & 0.013 & $\ldots$ & 0.000 \\
\hline 23344 & 0.312 & 0.354 & 0.065 & 2.617 & 1 & 0.014 & 0.360 & 0.360 & 0.049 & 0.036 & 0.039 & 0.028 & 0.010 & 0.090 \\
\hline 24316 & 0.371 & 0.205 & 0.060 & 2.567 & 1 & -0.001 & 0.503 & 0.486 & 0.027 & 0.020 & 0.014 & 0.010 & 0.000 & 0.010 \\
\hline 25659 & 0.365 & 0.170 & 0.081 & $\ldots$ & 1 & $\ldots$ & 0.510 & 0.508 & 0.018 & 0.013 & 0.023 & 0.017 & 0.000 & 0.015 \\
\hline 28887 & 0.455 & 0.232 & 0.102 & 2.547 & 1 & 0.039 & 0.676 & 0.645 & 0.351 & $\ldots$ & 0.120 & 0.088 & 0.020 & $\ldots$ \\
\hline 29759 & 0.435 & 0.218 & 0.070 & 2.553 & 1 & 0.038 & 0.611 & 0.596 & 0.282 & $\ldots$ & 0.072 & 0.053 & $\ldots$ & $\ldots$ \\
\hline 29992 & 0.559 & 0.364 & 0.137 & 2.532 & 2 & 0.072 & 0.829 & 0.840 & 0.227 & $\ldots$ & 0.026 & 0.019 & $\ldots$ & $\ldots$ \\
\hline 30098 & 0.506 & 0.228 & 0.123 & 2.547 & 1 & 0.080 & 0.750 & 0.740 & 0.210 & $\ldots$ & 0.212 & 0.155 & 0.010 & $\ldots$ \\
\hline 30668 & 0.451 & 0.277 & 0.108 & 2.544 & 1 & 0.025 & 0.667 & 0.664 & 0.819 & $\ldots$ & 0.036 & 0.026 & $\ldots$ & $\ldots$ \\
\hline 32567 & 0.322 & 0.304 & 0.078 & 2.614 & 1 & 0.002 & 0.444 & 0.430 & 0.057 & 0.041 & 0.077 & 0.056 & 0.010 & 0.090 \\
\hline 34630 & 0.343 & 0.255 & 0.053 & 2.596 & 1 & 0.009 & 0.480 & 0.440 & 0.073 & 0.053 & $\ldots$ & .. & 0.020 & 0.090 \\
\hline 36174 & 0.336 & 0.310 & 0.054 & 2.599 & 1 & 0.015 & 0.440 & 0.442 & 0.058 & 0.043 & 0.034 & 0.025 & 0.010 & 0.060 \\
\hline 36430 & 0.370 & 0.291 & 0.041 & 2.581 & 1 & 0.027 & 0.540 & 0.480 & 0.066 & 0.048 & 0.105 & 0.077 & 0.030 & 0.060 \\
\hline 36513 & 0.311 & 0.356 & 0.039 & 2.591 & 1 & -0.002 & 0.350 & 0.357 & 0.057 & 0.041 & 0.095 & 0.069 & 0.010 & 0.060 \\
\hline 37671 & 0.335 & 0.339 & 0.074 & 2.609 & 1 & 0.018 & 0.536 & 0.453 & 0.061 & 0.044 & 0.013 & 0.009 & 0.020 & 0.120 \\
\hline 38541 & 0.428 & 0.126 & 0.110 & 2.545 & 1 & 0.008 & 0.621 & 0.610 & 0.050 & 0.037 & 0.000 & 0.000 & 0.000 & 0.030 \\
\hline 40778 & 0.342 & 0244 & 0.072 & 2.586 & 1 & -0.010 & 0.484 & 0.476 & 0.044 & 0.032 & 0.022 & 0.016 & 0.000 & 0.020 \\
\hline 42592 & 0.311 & 0.295 & 0.067 & 2.596 & 1 & -0.017 & 0.431 & 0.421 & 0.053 & 0.038 & 0.032 & 0.023 & 0.000 & 0.060 \\
\hline 42887 & 0.237 & 0.489 & 0.103 & 2.677 & 1 & 0.004 & 0.316 & 0.307 & 0.030 & 0.022 & 0.011 & 0.008 & 0.000 & $\ldots$ \\
\hline 44605 & 0.371 & 0.141 & 0.062 & 2.576 & 1 & -0.003 & 0.527 & 0.506 & 0.022 & 0.016 & 0.022 & 0.016 & 0.000 & 0.015 \\
\hline 44716 & 0.522 & 0.440 & 0.168 & 2.547 & 2 & 0.019 & 0.822 & 0.796 & 0.041 & 0.030 & 0.024 & 0.018 & 0.020 & 0.015 \\
\hline 46516 & 0.306 & 0.378 & 0.055 & 2.614 & 1 & 0.017 & 0.390 & 0.385 & 0.046 & 0.034 & 0.004 & 0.003 & 0.020 & 0.015 \\
\hline 47480 & 0.299 & 0.348 & 0.056 & 2.613 & 1 & 0.004 & 0.405 & 0.375 & 0.100 & 0.073 & 0.007 & 0.005 & 0.020 & 0.060 \\
\hline 48152 & 0.303 & 0.354 & 0.056 & 2.613 & 1 & 0.009 & 0.421 & 0.393 & 0.042 & 0.031 & 0.004 & 0.003 & 0.010 & 0.015 \\
\hline 48444 & 0.515 & 0.360 & 0.030 & $\ldots$ & 2 & $\ldots$ & 0.835 & $\ldots$ & 0.011 & 0.008 & 0.072 & 0.053 & $\ldots$ & $\ldots$ \\
\hline 49371 & 0.479 & 0.278 & 0.116 & 2.539 & 1 & 0.073 & 0.719 & 0.700 & 0.006 & 0.005 & 0.037 & 0.027 & $\ldots$ & 0.015 \\
\hline
\end{tabular}


Table 4. continued.

\begin{tabular}{|c|c|c|c|c|c|c|c|c|c|c|c|c|c|c|}
\hline HIP & $(b-y)$ & $c 1$ & $m 1$ & $\beta$ & Ref. $^{a}$ & $\begin{array}{r}E(b-y) \\
\beta\end{array}$ & $\begin{array}{c}(B-V) \\
\text { Hip }\end{array}$ & $\begin{array}{c}(B-V) \\
\text { Lit }\end{array}$ & $\begin{array}{c}E(B-V) \\
\quad S 98\end{array}$ & $\begin{array}{c}E(b-y) \\
\text { S } 98\end{array}$ & $\begin{array}{c}E(B-V) \\
\text { H97 }\end{array}$ & $\begin{array}{c}E(b-y) \\
\text { H97 }\end{array}$ & $\begin{array}{c}E(B-V) \\
\quad \text { Lit }\end{array}$ & $\begin{array}{c}E(B-V) \\
\text { BH }\end{array}$ \\
\hline 49616 & 0.502 & 0.210 & 0.083 & 2.535 & 1 & 0.040 & 0.735 & 0.725 & 0.177 & 0.129 & 0.024 & 0.018 & 0.010 & 0.100 \\
\hline 52771 & 0.377 & 0.153 & 0.064 & 2.566 & 1 & -0.003 & 0.506 & 0.500 & 0.028 & 0.021 & 0.011 & 0.008 & 0.000 & 0.011 \\
\hline 53070 & 0.343 & 0.251 & 0.078 & 2.586 & 1 & -0.010 & 0.480 & 0.471 & 0.027 & 0.020 & 0.004 & 0.003 & 0.000 & 0.000 \\
\hline 54639 & 0.496 & 0.102 & 0.115 & 2.514 & 1 & 0.040 & 0.734 & 0.720 & 0.037 & 0.028 & 0.002 & 0.001 & 0.030 & \\
\hline 55022 & 0.293 & 0.407 & 0.104 & 2.638 & 1 & 0.002 & 0.425 & 0.417 & 0.050 & 0.037 & 0.002 & 0.001 & 0.000 & 0.015 \\
\hline 55852 & 0.523 & 0.242 & 0.178 & $\ldots$ & 2 & $\ldots$ & 0.820 & & 0.041 & 0.030 & $\ldots$ & $\ldots$ & . & 0.015 \\
\hline .44 & 0.402 & 0.154 & 0.069 & $\ldots$ & 2 & $\ldots$ & 0.535 & 0.535 & 0.013 & 0.010 & 0.016 & 0.012 & 0.000 & 0.015 \\
\hline 57983 & 0.476 & 0.209 & 0.120 & $\ldots$ & 2 & $\ldots$ & 0.725 & $\ldots$ & 0.023 & 0.016 & 0.031 & 0.023 & $\ldots$ & 0.015 \\
\hline 58357 & 0.527 & 0.306 & 0.273 & $\ldots$ & 3 & $\ldots$ & 0.860 & 0.860 & 0.029 & 0.021 & 0.027 & 0.020 & & 0.015 \\
\hline 59109 & 0.311 & 0.373 & 0.048 & 2.609 & 1 & 0.019 & 0.413 & 0.402 & 0.049 & 0.036 & 0.023 & 0.017 & 0.010 & 0.015 \\
\hline 59376 & 0.335 & 0.248 & 0.061 & 2.588 & 1 & -0.009 & 0.449 & 0.434 & 0.026 & 0.019 & 0.022 & 0.016 & 0.000 & 0.015 \\
\hline 60387 & 0.583 & 0.462 & 0.068 & $\ldots$ & 2 & 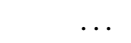 & 0.810 & 0.750 & 0.029 & 0.022 & 0.024 & 0.018 & & 0.015 \\
\hline 60632 & 0.330 & 0.287 & 0.059 & 2.594 & 1 & -0.001 & 0.445 & 0.432 & 0.023 & 0.016 & 0.015 & 0.011 & 0.000 & 0.015 \\
\hline 60719 & 0.448 & 0.306 & 0.053 & & 2 & & 0.631 & & 0.018 & 0.013 & 0.022 & 0.016 & & 0.015 \\
\hline 61361 & 0.332 & 0.225 & 0.054 & 2.593 & 1 & -0.009 & 0.448 & 0.423 & 0.037 & 0.027 & 0.021 & 0.015 & 0.000 & 0.015 \\
\hline 61545 & 0.321 & 0.301 & 0.058 & 2.595 & 1 & -0.005 & 0.448 & 0.408 & 0.018 & 0.013 & 0.011 & 0.008 & 0.000 & $\ldots$ \\
\hline 61802 & 0.433 & 0.236 & 0.137 & 2.555 & 1 & 0.017 & 0.652 & 0.653 & 0.040 & 0.029 & 0.022 & 0.016 & $\ldots$ & 0.015 \\
\hline 62747 & 0.511 & 0.296 & 0.177 & 2.549 & 1 & 0.065 & 0.800 & 0.799 & 0.052 & 0.038 & 0.006 & 0.004 & $\ldots$ & 0.015 \\
\hline 63385 & 0.516 & 0.351 & 0.091 & $\ldots$ & 2 & $\ldots$ & 0.749 & $\ldots$ & 0.022 & 0.016 & 0.011 & 0.008 & $\ldots$ & $\ldots$ \\
\hline 63559 & 0.376 & 0.250 & 0.110 & 2.598 & 1 & 0.011 & 0.550 & 0.544 & 0.080 & 0.059 & 0.000 & 0.000 & 0.010 & 0.090 \\
\hline 64786 & 0.586 & 0.467 & 0.056 & $\ldots$ & 2 & $\ldots$ & 0.792 & 0.784 & 0.014 & 0.010 & 0.024 & 0.018 & & \\
\hline 65201 & 0.349 & 0.278 & 0.050 & & 1 & & 0.450 & 0.468 & 0.074 & 0.054 & 0.000 & 0.000 & 0.010 & 0.060 \\
\hline 65206 & 0.366 & 0.208 & 0.062 & $\ldots$ & 3 & $\ldots$ & 0.480 & 0.483 & 0.024 & 0.018 & 0.041 & 0.030 & 0.000 & 0.015 \\
\hline 66354 & 0.430 & 0.190 & 0.167 & 2.553 & 1 & 0.002 & 0.670 & 0.667 & 0.025 & 0.018 & 0.006 & 0.004 & 0.000 & 0.015 \\
\hline 66673 & 0.312 & 0.336 & 0.037 & 2.622 & 1 & 0.029 & 0.405 & 0.381 & 0.028 & 0.020 & 0.011 & 0.008 & 0.000 & 0.015 \\
\hline 67655 & 0.424 & 0.207 & 0.173 & 2.567 & 1 & 0.005 & 0.663 & 0.662 & 0.692 & $\ldots$ & 0.013 & 0.009 & 0.010 & $\ldots$ \\
\hline 67863 & 0.395 & 0.268 & 0.140 & 2.588 & 1 & 0.012 & 0.605 & 0.604 & 0.113 & 0.083 & 0.035 & 0.026 & 0.010 & 0.120 \\
\hline 68321 & 0.309 & 0.359 & 0.056 & 2.602 & 1 & 0.003 & 0.410 & 0.404 & 0.015 & 0.011 & 0.016 & 0.012 & 0.010 & 0.015 \\
\hline 68464 & 0.343 & 0.340 & 0.055 & 2.597 & 2 & 0.023 & 0.463 & 0.462 & 0.092 & 0.067 & 0.156 & 0.114 & 0.010 & 0.060 \\
\hline 68592 & 0.299 & 0.329 & 0.057 & 2.626 & 1 & 0.009 & 0.397 & 0.370 & 0.028 & 0.020 & 0.006 & 0.004 & 0.020 & 0.040 \\
\hline 70647 & 0.597 & 0.392 & 0.050 & & 2 & & 0.824 & 0.818 & 0.099 & 0.074 & 0.012 & 0.009 & & \\
\hline 71087 & 0.506 & 0.382 & 0.115 & $\ldots$ & 2 & & 0.818 & $\cdots$ & 0.020 & 0.015 & 0.011 & 0.008 & $\ldots$ & $\ldots$ \\
\hline 71458 & 0.466 & 0.261 & 0.056 & 2.545 & 2 & 0.057 & 0.660 & 0.638 & 0.099 & 0.073 & 0.652 & 0.476 & $\ldots$ & 0.090 \\
\hline 72461 & 0.336 & 0.280 & 0.050 & 2.612 & 1 & 0.020 & 0.437 & 0.425 & 0.031 & 0.023 & 0.011 & 0.008 & 0.020 & 0.015 \\
\hline 72561 & 0.305 & 0.358 & 0.072 & 2.634 & 1 & 0.018 & 0.413 & 0.399 & 0.044 & 0.032 & 0.006 & 0.004 & 0.030 & 0.030 \\
\hline 72920 & 0.324 & 0.322 & 0.034 & 2.619 & 4 & 0.035 & 0.400 & 0.410 & 0.033 & 0.024 & 0.011 & 0.008 & 0.000 & 0.015 \\
\hline 73385 & 0.401 & 0.285 & 0.063 & 2.578 & 1 & 0.041 & 0.554 & 0.557 & 0.111 & 0.081 & 0.011 & 0.008 & $\ldots$ & 0.010 \\
\hline 74079 & 0.370 & 0.312 & 0.119 & 2.582 & 2 & -0.003 & 0.565 & 0.535 & 0.041 & 0.030 & 0.006 & 0.004 & $\ldots$ & $\ldots$ \\
\hline 74234 & 0.524 & 0.173 & 0.297 & 2.523 & 1 & 0.029 & 0.850 & 0.853 & 0.088 & 0.065 & 0.013 & 0.009 & 0.000 & 0.110 \\
\hline 74235 & 0.484 & 0.165 & 0.224 & 2.528 & 1 & 0.014 & 0.770 & 0.775 & 0.086 & 0.063 & 0.013 & 0.009 & $\cdots$ & 0.110 \\
\hline 76059 & 0.422 & 0.170 & 0.065 & 2.565 & 1 & 0.037 & 0.600 & 0.565 & 0.187 & 0.137 & 0.011 & 0.008 & $\ldots$ & 0.150 \\
\hline 76976 & 0.380 & 0.284 & 0.033 & 2.564 & 1 & 0.015 & 0.520 & 0.490 & 0.164 & 0.120 & 0.012 & 0.009 & $\ldots$ & 0.180 \\
\hline 78640 & 0.340 & 0.271 & 0.071 & 2.582 & 1 & -0.011 & 0.481 & 0.470 & 0.013 & 0.009 & 0.025 & 0.018 & 0.000 & 0.015 \\
\hline 79537 & 0.509 & 0.172 & 0.282 & 2.534: & 1 & 0.026 & 0.815 & 0.819 & 0.484 & $\ldots$ & 0.010 & 0.007 & 0.020 & $\ldots$ \\
\hline 80679 & 0.488 & 0.104 & 0.072 & 2.527 & 1 & 0.060 & 0.740 & 0.683 & 0.064 & 0.047 & 0.018 & 0.013 & 0.000 & $\ldots$ \\
\hline 81170 & 0.476 & 0.162 & 0.202 & 2.528 & 1 & 0.012 & 0.736 & 0.741 & 0.401 & $\ldots$ & 0.046 & 0.034 & $\cdots$ & 0.180 \\
\hline 81276 & 0.271 & 0.401 & 0.087 & 2.616 & $1 \mathrm{a}$ & -0.024 & 0.360 & 0.360 & 0.015 & 0.011 & 0.031 & 0.023 & 0.000 & 0.000 \\
\hline 83320 & 0.375 & 0.261 & 0.029 & 2.582 & $1 \mathrm{a}$ & 0.034 & 0.460 & 0.475 & 0.098 & 0.071 & 0.022 & 0.016 & 0.060 & 0.090 \\
\hline 85855 & 0.461 & 0.275 & 0.061 & 2.538 & $1 \mathrm{a}$ & 0.037 & 0.607 & 0.640 & 0.010 & 0.047 & 0.070 & 0.051 & 0.000 & 0.080 \\
\hline 86443 & 0.356 & 0.251 & 0.047 & 2.574 & 1 & 0.001 & 0.458 & 0.450 & 0.214 & $\ldots$ & 0.072 & 0.053 & 0.010 & 0.150 \\
\hline
\end{tabular}


Table 4. continued.

\begin{tabular}{|c|c|c|c|c|c|c|c|c|c|c|c|c|c|c|}
\hline HIP & $(b-y)$ & $c 1$ & $m 1$ & $\beta$ & $\operatorname{Ref}^{a}$ & $\begin{array}{r}E(b-y) \\
\beta\end{array}$ & $\begin{array}{c}(B-V) \\
\text { Hip }\end{array}$ & $\begin{array}{c}(B-V) \\
\text { Lit }\end{array}$ & $\begin{array}{c}E(B-V) \\
\quad \mathrm{S} 98\end{array}$ & $\begin{array}{c}E(b-y) \\
\text { S } 98\end{array}$ & $\begin{array}{c}E(B-V) \\
\text { H97 }\end{array}$ & $\begin{array}{c}E(b-y) \\
\text { H97 }\end{array}$ & $\begin{array}{c}E(B-V) \\
\quad \text { Lit }\end{array}$ & $\begin{array}{c}E(B-V) \\
\text { BH }\end{array}$ \\
\hline 86694 & 0.347 & 0.331 & 0.051 & 2.636 & 1 & 0.055 & 0.456 & 0.456 & 0.525 & $\cdots$ & 0.051 & 0.037 & & $\ldots$ \\
\hline 87062 & 0.452 & 0.274 & 0.032 & 2.587 & 1 & 0.105 & 0.605 & 0.601 & 0.919 & - & 0.142 & 0.104 & 0.100 & $\ldots$ \\
\hline 87101 & 0.489 & 0.301 & 0.036 & 2.568 & 1 & 0.117 & 0.672 & 0.665 & 0.922 & & 0.059 & 0.043 & & $\ldots$ \\
\hline 87467 & 0.323 & 0.329 & 0.042 & 2.615 & $1 \mathrm{a}$ & 0.028 & 0.520 & 0.420 & 0.041 & 0.040 & 0.037 & 0.027 & 0.010 & 0.030 \\
\hline 87693 & 0.319 & 0.310 & 0.050 & 2.602 & 1 & 0.006 & 0.442 & 0.440 & 0.092 & 0.067 & 0.063 & 0.046 & 0.010 & 0.090 \\
\hline 88010 & 0.423 & 0.199 & 0.114 & 2.558 & 1 & 0.017 & 0.616 & 0.606 & 0.888 & - & 0.052 & 0.038 & 0.030 & $\ldots$ \\
\hline 88827 & 0.362 & 0.374 & 0.034 & 2.627 & 1 & 0.084 & 0.454 & 0.455 & 0.308 & & 0.137 & 0.100 & 0.080 & $\ldots$ \\
\hline 89554 & 0.327 & 0.309 & 0.074 & 2.610 & 2 & 0.007 & 0.460 & 0.451 & 0.117 & 0.085 & 0.035 & 0.026 & $\ldots$ & 0.100 \\
\hline 90957 & 0.505 & 0.259 & 0.006 & 2.564 & 1 & 0.136 & 0.667 & 0.649 & 0.294 & & 0.119 & 0.087 & & $\ldots$ \\
\hline 91129 & 0.340 & 0.261 & 0.029 & 2.617 & 1 & 0.031 & 0.450 & 0.416 & 0.185 & 0.135 & 0.047 & 0.034 & 0.050 & 0.180 \\
\hline 92167 & 0.504 & 0.290 & 0.152 & 2.541 & 2 & 0.055 & 0.763 & 0.754 & 0.109 & 0.079 & 0.133 & 0.097 & $\ldots$ & 0.060 \\
\hline 92775 & 0.430 & 0.231 & 0.100 & $\ldots$ & 3 & $\ldots$ & 0.680 & 0.610 & 0.086 & 0.063 & 0.081 & 0.059 & $\ldots$ & 0.070 \\
\hline 95333 & 0.351 & 0.224 & 0.052 & 2.582 & 1 & 0.000 & 0.473 & 0.461 & 0.091 & 0.066 & 0.009 & 0.007 & $\cdots$ & 0.060 \\
\hline 96115 & 0.308 & 0.366 & 0.045 & 2.600 & 1 & 0.006 & 0.399 & 0.389 & 0.975 & & 0.062 & 0.045 & 0.020 & \\
\hline 98020 & 0.416 & 0.163 & 0.100 & 2.553 & 1 & 0.009 & 0.599 & 0.592 & 0.279 & $\ldots$ & 0.029 & 0.021 & 0.000 & $\ldots$ \\
\hline 98532 & 0.386 & 0.284 & 0.111 & 2.572 & 2 & 0.003 & 0.575 & 0.565 & 0.246 & $\cdots$ & 0.037 & 0.027 & $\ldots$ & 0.120 \\
\hline 98974 & 0.506 & 0.305 & 0.134 & $\ldots$ & 2 & - & 0.753 & 0.750 & 0.114 & 0.083 & 0.055 & 0.040 & $\ldots$ & 0.080 \\
\hline 98989 & 0.363 & 0.271 & 0.057 & 2.585 & 1 & 0.017 & 0.510 & 0.471 & 0.137 & 0.100 & 0.092 & 0.067 & 0.030 & 0.150 \\
\hline 99267 & 0.377 & 0.185 & 0.053 & $\ldots$ & 3 & $\ldots$ & 0.510 & 0.510 & 1.181 & $\ldots$ & 0.022 & 0.016 & 0.040 & $\ldots$ \\
\hline 99423 & 0.372 & 0.289 & 0.078 & 2.576 & 1 & 0.008 & 0.554 & 0.510 & 0.832 & $\ldots$ & 0.040 & 0.029 & $\ldots$ & $\ldots$ \\
\hline 100568 & 0.383 & 0.221 & 0.099 & 2.568 & 1 & -0.003 & 0.554 & 0.549 & 0.060 & 0.044 & 0.051 & 0.037 & 0.000 & 0.030 \\
\hline 100682 & 0.306 & 0.339 & 0.041 & 2.608 & 1 & 0.011 & 0.427 & 0.397 & 0.400 & 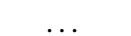 & 0.048 & 0.035 & 0.020 & $\ldots$ \\
\hline 100792 & 0.344 & 0.269 & 0.091 & 2.588 & 1 & -0.011 & 0.503 & 0.483 & 0.102 & 0.074 & 0.006 & 0.004 & 0.000 & 0.120 \\
\hline 102337 & 0.323 & 0.344 & 0.047 & 2.606 & 1 & 0.021 & 0.428 & 0.418 & 0.073 & 0.053 & 0.020 & 0.015 & 0.040 & 0.060 \\
\hline 102718 & 0.359 & 0.260 & 0.080 & 2.581 & 1 & -0.001 & 0.520 & 0.510 & 0.090 & 0.066 & 0.068 & 0.050 & 0.010 & 0.060 \\
\hline 103269 & 0.417 & 0.108 & 0.115 & 2.553 & 1a & 0.002 & 0.590 & 0.613 & 2.705 & & 0.027 & 0.020 & 0.030 & $\cdots$ \\
\hline 103337 & 0.552 & 0.363 & 0.097 & $\cdots$ & 2 & $\ldots$ & 0.893 & $\cdots$ & 0.035 & 0.025 & 0.034 & 0.025 & $\ldots$ & 0.040 \\
\hline 104191 & 0.460 & 0.271 & 0.027 & 2.534 & 1 & 0.037 & 0.640 & 0.630 & 0.029 & 0.021 & 0.018 & 0.013 & 0.000 & 0.000 \\
\hline 104660 & 0.394 & 0.280 & 0.141 & 2.572 & 1 & -0.002 & 0.595 & 0.588 & 0.144 & 0.086 & 0.051 & 0.037 & $\ldots$ & 0.100 \\
\hline 104658 & 0.363 & 0.261 & 0.094 & 2.586 & 1 & 0.001 & 0.110 & 0.510 & 0.117 & 0.105 & 0.011 & 0.008 & 0.000 & 0.150 \\
\hline 106468 & 0.321 & 0.293 & 0.040 & 2.603 & 1 & 0.010 & 0.405 & 0.450 & 0.063 & 0.046 & 0.022 & 0.016 & 0.010 & 0.030 \\
\hline 108200 & 0.464 & 0.130 & 0.111 & 2.523 & 1 & 0.021 & 0.686 & 0.650 & 0.114 & 0.083 & 0.018 & 0.013 & 0.010 & 0.120 \\
\hline 109558 & 0.330 & 0.327 & 0.063 & 2.588 & 1 & -0.004 & 0.459 & 0.442 & 0.053 & 0.039 & 0.023 & 0.017 & 0.010 & 0.060 \\
\hline 110140 & 0.346 & 0.286 & 0.073 & 2.581 & 1 & -0.007 & 0.489 & 0.460 & 0.072 & 0.052 & 0.028 & 0.020 & 0.020 & 0.060 \\
\hline 111195 & 0.366 & 0.275 & 0.075 & 2.586 & 1 & 0.014 & 0.520 & 0.485 & 0.077 & 0.056 & 0.019 & 0.014 & 0.020 & 0.040 \\
\hline 111372 & 0.322 & 0.337 & 0.050 & 2.597 & 1 & 0.007 & 0.432 & 0.418 & 0.013 & 0.010 & 0.018 & 0.013 & 0.000 & 0.015 \\
\hline 114271 & 0.312 & 0.361 & 0.059 & 2.616 & 2 & 0.018 & 0.411 & $\ldots$ & 0.034 & 0.025 & 0.011 & 0.008 & 0.010 & 0.015 \\
\hline 114502 & 0.501 & 0.330 & 0.075 & $\ldots$ & 2 & $\ldots$ & 0.718 & 0.720 & 0.036 & 0.026 & 0.011 & 0.008 & $\ldots$ & 0.015 \\
\hline 114661 & 0.478 & 0.078 & 0.061 & 2.551 & 1 & 0.068 & 0.690 & 0.657 & 0.141 & 0.104 & 0.024 & 0.018 & 0.010 & 0.015 \\
\hline 114962 & 0.349 & 0.243 & 0.072 & 2.584 & 1 & -0.006 & 0.481 & 0.475 & 0.033 & 0.024 & 0.011 & 0.008 & 0.000 & 0.015 \\
\hline 115167 & 0.339 & 0.332 & 0.059 & 2.590 & 1 & 0.008 & 0.456 & 0.442 & 0.061 & 0.045 & 0.023 & 0.017 & 0.020 & 0.015 \\
\hline 115704 & 0.330 & 0.256 & 0.064 & 2.618 & 1 & 0.005 & 0.469 & 0.480 & 1.337 & & 0.070 & 0.051 & 0.030 & $\cdots$ \\
\hline 117522 & 0.399 & 0.124 & 0.045 & 2.542 & 2 & 0.017 & 0.532 & 0.534 & 0.100 & 0.073 & 0.021 & 0.015 & 0.020 & 0.030 \\
\hline
\end{tabular}

${ }^{a}$ References: 1. Schuster \& Nissen (1988); 1a. Schuster (2002) (priv. comm.); 2. Hauck \& Mermilliod (1998); 3. Laird et al. (1988); 4. Ryan et al. (1999). 
Table 5. Final temperatures as derived from different reddening cor- Table 5. continued. rections.

\begin{tabular}{|c|c|c|c|c|c|c|}
\hline \multirow[t]{2}{*}{ HIP } & \multicolumn{6}{|c|}{ Effective temperature scales (K) } \\
\hline & 2 & 1 & 3 & 4 & S98 & H97 \\
\hline \multicolumn{7}{|c|}{ Sample \#1: the clean sample } \\
\hline 911 & 5972 & 5918 & 5972 & 5972 & 6076 & 6065 \\
\hline 3026 & 6040 & 6026 & 6040 & 6040 & 6221 & 6214 \\
\hline 3446 & 5901 & 5901 & 5894 & 5894 & 5991 & 5995 \\
\hline 3564 & 5683 & 5683 & 5683 & 5683 & 5974 & 5679 \\
\hline 8572 & 6287 & 6287 & 6287 & 6287 & 6265 & 6287 \\
\hline 10140 & 5608 & 5530 & 5608 & 5608 & 6085 & 5698 \\
\hline 11952 & 6142 & 6142 & 6107 & 6142 & 6222 & 6158 \\
\hline 12529 & 6057 & 6057 & 6057 & 6057 & & 6069 \\
\hline 14594 & 5879 & 5872 & 5879 & 5879 & 6867: & 5936 \\
\hline 16072 & 5933 & 5933 & 5933 & 5933 & $\ldots$ & 5849 \\
\hline 18082 & 5933 & 5933 & 5933 & 5933 & $\ldots$ & 5782 \\
\hline 18802 & 5756 & 5656 & 5756 & 5756 & 5819 & 5834 \\
\hline 19797 & 6044 & 5975 & 6044 & 6044 & & 6143 \\
\hline 22632 & 5882 & 5882 & 5856 & 5856 & 6038 & 5945 \\
\hline 23344 & 6346 & 6346 & 6346 & 6346 & 6516 & 6401 \\
\hline 24316 & 5724 & 5717 & 5724 & 5724 & 5854 & 5792 \\
\hline 28887 & 5415 & 5415 & 5415 & 5415 & $\ldots$ & 5719 \\
\hline 29759 & 5521 & 5521 & 5521 & 5521 & $\ldots$ & 5602 \\
\hline 30098 & 5363 & 5363 & 5363 & 5363 & $\ldots$ & 5859 \\
\hline 32567 & 6112 & 6112 & 6098 & 6112 & 6396 & 6512 \\
\hline 34630 & 6033 & 6033 & 5970 & 6033 & 6358 & \\
\hline 36174 & 6110 & 6110 & 6110 & 6110 & 6312 & 6195 \\
\hline 36430 & 5985 & 5985 & 5985 & 5985 & 6133 & 6301 \\
\hline 36513 & 6250 & 6235 & 6250 & 6250 & 6564 & 6749 \\
\hline 37671 & 6149 & 6149 & 6149 & 6149 & 6337 & 6091 \\
\hline 38541 & 5384 & 5384 & 5384 & 5337 & 5560 & 5329 \\
\hline 40778 & 5935 & 5868 & 5935 & 5935 & 6159 & 6039 \\
\hline 42592 & 6203 & 6081 & 6203 & 6203 & 6491 & 6337 \\
\hline 44605 & 5727 & 5704 & 5723 & 5723 & 5830 & 5799 \\
\hline 44716 & 5045 & 5045 & 5045 & 5045 & 5094 & 5040 \\
\hline 46516 & 6426 & 6426 & 6426 & 6426 & 6559 & 6320 \\
\hline 47480 & 6367 & 6367 & 6337 & 6367 & 6931 & 6362 \\
\hline 48152 & 6377 & 6377 & 6309 & 6377 & 6548 & 6329 \\
\hline 52771 & 5669 & 5650 & 5669 & 5669 & 5807 & 5714 \\
\hline 53070 & 5930 & 5864 & 5930 & 5930 & 6068 & 5946 \\
\hline 55022 & 6367 & 6367 & 6352 & 6367 & 6632 & 6361 \\
\hline 59109 & 6400 & 6400 & 6400 & 6400 & 6532 & 6385 \\
\hline 59376 & 6024 & 5961 & 6024 & 6024 & 6160 & 6095 \\
\hline 60632 & 6044 & 6037 & 6044 & 6044 & 6157 & 6123 \\
\hline 61361 & 6034 & 5971 & 6034 & 6034 & 6231 & 6103 \\
\hline 61545 & 6150 & 6114 & 6150 & 6150 & 6245 & 6179 \\
\hline 61802 & 5455 & 5455 & 5455 & 5455 & 5525 & 5454 \\
\hline 62747 & 5278 & 5278 & 5278 & 5278 & 5150 & 5021 \\
\hline 63559 & 5786 & 5786 & 5786 & 5710 & 6106 & 5644 \\
\hline 66354 & 5362 & 5362 & 5362 & 5362 & 5455 & 5379 \\
\hline 66673 & 6452 & 6452 & 6452 & 6452 & 6383 & 6292 \\
\hline 67655 & 5429 & 5429 & 5400 & 5400 & $\ldots$ & 5461 \\
\hline 67863 & 5683 & 5683 & 5683 & 5610 & 6150 & 5789 \\
\hline 68321 & 6288 & 6288 & 6266 & 6288 & 6349 & 6345 \\
\hline
\end{tabular}

\begin{tabular}{rcccccc}
\hline \hline HIP & \multicolumn{7}{c}{ Effective temperature scales (K) } \\
& 2 & 1 & 3 & 4 & S98 & H97 \\
\hline \multicolumn{7}{c}{ Sample \#1: the clean sample } \\
\hline 68464 & 6156 & 6156 & 6156 & 6156 & 6485 & 6820 \\
68592 & 6396 & 6396 & 6328 & 6396 & 6482 & 6336 \\
71458 & 5457 & 5457 & 5457 & 5457 & 5546 & $\ldots$ \\
72461 & 6175 & 6175 & 6175 & 6175 & 6197 & 6072 \\
72561 & 6388 & 6388 & 6388 & 6388 & 6495 & 6285 \\
72920 & 6398 & 6398 & 6398 & 6398 & 6314 & 6196 \\
73385 & 5831 & 5831 & 5831 & 5831 & 6102 & 5635 \\
74079 & 5781 & 5762 & 5781 & 5781 & 5979 & 5811 \\
76059 & 5617 & 5617 & 5617 & 5735 & $6310:$ & 5444 \\
76976 & 5832 & 5832 & 5832 & 5832 & $6607:$ & 5792 \\
78640 & 5960 & 5887 & 5960 & 5960 & 6022 & 6080 \\
81276 & 6523 & 6343 & 6523 & 6523 & 6608 & 6693 \\
86443 & 5885 & 5885 & 5878 & 5885 & $\ldots$ & 6209 \\
86694 & 6341 & 6341 & 6341 & 6341 & $\ldots$ & 6216 \\
87101 & 5660 & 5660 & 5660 & 5660 & $\ldots$ & 5262 \\
87467 & 6356 & 6356 & 6356 & 6356 & 6448 & 6348 \\
87693 & 6212 & 6212 & 6168 & 6212 & 6688 & 6466 \\
88010 & 5496 & 5496 & 5496 & 5496 & $\ldots$ & 5625 \\
88827 & 6504 & 6504 & 6504 & 6504 & $\ldots$ & 6591 \\
89554 & 6120 & 6120 & 6071 & 6071 & 6714 & 6246 \\
95333 & 5869 & 5869 & 5869 & 5869 & 6341 & 5923 \\
96115 & 6322 & 6322 & 6277 & 6322 & $\ldots$ & 6603 \\
98020 & 5480 & 5480 & 5426 & 5426 & $\ldots$ & 5545 \\
98532 & 5680 & 5680 & 5661 & 5680 & $\ldots$ & 5842 \\
98989 & 5925 & 5925 & 5925 & 5925 & $6533:$ & 6261 \\
99423 & 5806 & 5806 & 5755 & 5806 & $\ldots$ & 5941 \\
100568 & 5651 & 5632 & 5651 & 5651 & 5934 & 5892 \\
100682 & 6362 & 6362 & 6362 & 6362 & $\ldots$ & 6538 \\
100792 & 5858 & 5931 & 5931 & 5931 & 6466 & 5954 \\
102337 & 6305 & 6305 & 6305 & 6305 & 6552 & 6265 \\
102718 & 5832 & 5832 & 5832 & 5832 & 6304 & 6165 \\
104660 & 5626 & 5614 & 5626 & 5626 & $6325:$ & 5885 \\
104658 & 5804 & 5804 & 5798 & 5804 & 6401 & 5849 \\
106468 & 6219 & 6219 & 6219 & 6219 & 6495 & 6263 \\
109558 & 6037 & 6065 & 6065 & 6065 & 6346 & 6184 \\
110140 & 5922 & 5875 & 5922 & 5922 & 6284 & 6058 \\
111195 & 5875 & 5875 & 5875 & 5875 & 6164 & 5877 \\
111372 & 6209 & 6209 & 6158 & 6209 & 6230 & 6252 \\
114271 & 6349 & 6349 & 6349 & 6218 & 6402 & 6293 \\
114962 & 5883 & 5843 & 5883 & 5883 & 6046 & 5937 \\
115167 & 6065 & 6065 & 6010 & 6065 & 6332 & 6131 \\
115704 & 6088 & 6088 & 6053 & 6088 & $\ldots$ & 6383 \\
\hline & & & & & & \\
\hline \multicolumn{7}{c}{ (1) }
\end{tabular}


C. Charbonnel and F. Primas: The lithium content of the Galactic Halo stars, Online Material p 9

Table 5. continued.

\begin{tabular}{|c|c|c|c|c|c|c|}
\hline \multirow[t]{2}{*}{ HIP } & \multicolumn{6}{|c|}{ Effective temperature scales (K) } \\
\hline & 2 & 1 & 3 & 4 & S98 & H97 \\
\hline \multicolumn{7}{|c|}{ Sample \#2: the $\beta$ sample } \\
\hline 484 & 5064 & 5064 & 5064 & 5064 & 5064 & \\
\hline 3554 & 5008 & 5008 & 5008 & 5008 & 5040 & 5022 \\
\hline 4343 & 5064 & 5064 & 5064 & 5064 & 5069 & 5064 \\
\hline 8314 & 6430 & 6430 & 6430 & 6430 & & 6444 \\
\hline 13749 & 4965 & 4965 & 4965 & 4965 & 4996 & 4965 \\
\hline 17001 & 5226 & 5226 & 5226 & 5226 & 5231 & 5226 \\
\hline 21609 & 5247 & 5247 & 5247 & 5236 & 5392 & 5247 \\
\hline 25659 & 5855 & 5855 & 5855 & 5855 & 5856 & 5853 \\
\hline 30668 & 5386 & 5386 & 5386 & 5386 & 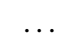 & 5386 \\
\hline 49371 & 5168 & 5168 & 5168 & 5168 & 5125 & 5231 \\
\hline 57244 & 5586 & 5586 & 5586 & 5586 & 5592 & 5580 \\
\hline 57983 & 5196 & 5196 & 5196 & 5196 & 5191 & 5238 \\
\hline 58357 & 4965 & 4965 & 4965 & 4965 & 4978 & 5129 \\
\hline 60719 & 5319 & 5319 & 5319 & 5319 & 5319 & 5334 \\
\hline 63385 & 4981 & 4981 & 4981 & 4981 & 5001 & 4961 \\
\hline 65206 & 5925 & 5925 & 5925 & 5925 & 5911 & 5953 \\
\hline 92775 & 5762 & 5762 & 5762 & 5762 & 5788 & 5729 \\
\hline 99267 & 5858 & 5858 & 5858 & 5858 & $\ldots$ & 5784 \\
\hline 114502 & 5065 & 5065 & 5065 & 5065 & 5117 & 5031 \\
\hline 117522 & 5648 & 5648 & 5615 & 5648 & 6023 & 5613 \\
\hline \multicolumn{7}{|c|}{ Sample \#3: the $u b v y$ sample } \\
\hline 12807 & 5932 & 5932 & 5932 & 5932 & 6872: & 5959 \\
\hline 83320 & 5984 & 5984 & 5984 & 5984 & 6251 & 5861 \\
\hline 87062 & 5909 & 5909 & 5909 & 5909 & $\cdots$ & 5905 \\
\hline 91129 & 6217 & 6217 & 6217 & 6217 & 7081: & 6237 \\
\hline 103337 & 4885 & 4885 & 4885 & 4885 & 4876 & 4876 \\
\hline 104191 & 5277 & 5277 & 5277 & 5277 & 5298 & 5257 \\
\hline
\end{tabular}


Table 6. Final Li abundances as derived by using different sets of $T_{\text {eff }}$.

\begin{tabular}{|c|c|c|c|c|c|c|c|c|c|c|c|c|}
\hline$\overline{\mathrm{HIP}}$ & Bin & $\begin{array}{c}\mathrm{Fe} / \mathrm{H}] \\
\operatorname{dex}\end{array}$ & $\begin{array}{c}\sigma(\mathrm{Fe}) \\
\operatorname{dex}\end{array}$ & $\begin{array}{c}\log g \\
\mathrm{~cm} \mathrm{~s}^{-1}\end{array}$ & $\begin{array}{l}E W \\
\mathrm{~mA}\end{array}$ & $\begin{array}{l} \pm 1 \sigma \\
\mathrm{mA}\end{array}$ & $\begin{array}{c}A(\mathrm{Li})_{\text {LTE }} \\
\operatorname{dex}\end{array}$ & $\begin{array}{c}A(\mathrm{Li})_{\text {NLTE }} \\
\text { dex }\end{array}$ & $\begin{array}{c}+1 \sigma \\
\operatorname{dex}\end{array}$ & $\begin{array}{c}-1 \sigma \\
\operatorname{dex}\end{array}$ & $\begin{array}{c}A(\mathrm{Li})_{\mathrm{S} 98} \\
\text { dex }\end{array}$ & $\begin{array}{c}A(\mathrm{Li})_{\mathrm{H} 97} \\
\text { dex }\end{array}$ \\
\hline \multicolumn{13}{|c|}{ Sample \#1: the clean sample } \\
\hline 911 & $\mathrm{~s}$ & -1.84 & 0.150 & 4.50 & 30.8 & 3.5 & 2.117 & 2.112 & 0.042 & 0.067 & 2.200 & 2.191 \\
\hline 3026 & $\mathrm{x}$ & -1.25 & 0.070 & 3.85 & 45.0 & 6.0 & 2.428 & 2.418 & 0.078 & 0.086 & 2.573 & 2.567 \\
\hline 3446 & & -3.50 & 0.100 & 4.50 & 27.0 & 3.9 & 1.973 & 1.986 & 0.074 & 0.076 & 2.045 & 2.048 \\
\hline 3564 & & -1.27 & 0.150 & 3.50 & 35.2 & 3.5 & 2.011 & 2.054 & 0.056 & 0.076 & 2.244 & 2.008 \\
\hline 8572 & & -2.51 & 0.010 & 3.85 & 27.0 & 1.4 & 2.257 & 2.236 & 0.026 & 0.027 & 2.239 & 2.257 \\
\hline 10140 & & -1.00 & 0.150 & 4.20 & 14.0 & 3.0 & 1.479 & 1.536 & 0.092 & 0.113 & 1.861 & 1.551 \\
\hline 11952 & & -1.85 & 0.170 & 4.50 & 28.0 & 2.6 & 2.183 & 2.162 & 0.052 & 0.048 & 2.247 & 2.196 \\
\hline 12529 & $*$ & -2.25 & 0.070 & 3.85 & 34.0 & 1.5 & 2.227 & 2.222 & 0.046 & 0.024 & 2.227 & 2.237 \\
\hline 14594 & $\mathrm{v}$ & -2.06 & 0.060 & 4.50 & 37.5 & 3.5 & 2.178 & 2.177 & 0.052 & 0.075 & 2.178 & 2.224 \\
\hline 16072 & & -1.35 & 0.150 & 4.50 & 44.2 & 3.5 & 2.315 & 2.308 & 0.047 & 0.049 & 2.341 & 2.274 \\
\hline 18082 & & -1.97 & 0.270 & 3.50 & 47.7 & 3.0 & 2.376 & 2.364 & 0.038 & 0.040 & 2.386 & 2.255 \\
\hline 18802 & & -1.06 & 0.100 & 3.50 & 23.0 & 2.0 & 1.853 & 1.894 & 0.042 & 0.045 & 1.903 & 1.915 \\
\hline 19797 & $\mathrm{x}$ & -1.58 & 0.050 & 4.00 & 27.9 & 2.8 & 2.139 & 2.131 & 0.079 & 0.054 & 2.133 & 2.211 \\
\hline 22632 & & -1.40 & 0.100 & 4.50 & 35.0 & 3.5 & 2.165 & 2.169 & 0.056 & 0.077 & 2.290 & 2.215 \\
\hline 23344 & $?$ & -2.77 & 0.080 & 4.00 & 20.5 & 1.1 & 2.155 & 2.134 & 0.026 & 0.026 & 2.291 & 2.199 \\
\hline 24316 & & -1.61 & 0.090 & 4.50 & 32.3 & 2.9 & 1.982 & 2.005 & 0.066 & 0.047 & 2.086 & 2.036 \\
\hline 28887 & & -1.67 & 0.030 & 3.00 & 18.0 & 2.0 & 1.418 & 1.511 & 0.051 & 0.057 & 1.418 & 1.693 \\
\hline 29759 & $?$ & -2.03 & 0.170 & 3.50 & 13.7 & 2.1 & 1.370 & 1.443 & 0.067 & 0.078 & 1.370 & 1.435 \\
\hline 30098 & & -1.98 & 0.150 & 3.20 & 16.0 & 3.0 & 1.308 & 1.407 & 0.083 & 0.100 & 1.308 & 1.698 \\
\hline 32567 & & -1.46 & 0.030 & 3.85 & 34.5: & 2.4 & 2.321: & 2.310: & 0.040 & 0.065 & 2.548: & 2.641: \\
\hline 34630 & $*$ & -2.53 & 0.240 & 4.20 & 30.0 & 2.9 & 2.128 & 2.124 & 0.049 & 0.052 & 2.385 & $\ldots$ \\
\hline 36174 & & -1.56 & 0.090 & 4.20 & 35.8: & 2.7 & 2.342: & 2.325: & 0.044 & 0.070 & 2.504: & 2.410: \\
\hline 36430 & $\mathrm{x}$ & -2.10 & 0.210 & 3.50 & 44.0 & 3.0 & 2.356 & 2.352 & 0.040 & 0.042 & 2.474 & 2.609 \\
\hline 36513 & ? & -2.65 & 0.060 & 3.85 & 24.0 & 4.0 & 2.159 & 2.145 & 0.078 & 0.094 & 2.410 & 2.571 \\
\hline 37671 & $\mathrm{~s}$ & -1.48 & 0.150 & 3.85 & 37.6 & 3.3 & 2.398 & 2.380 & 0.051 & 0.082 & 2.548 & 2.352 \\
\hline 38541 & $?$ & -1.77 & 0.050 & 4.50 & 12.0 & 2.0 & 1.206 & 1.274 & 0.072 & 0.084 & 1.347 & 1.079 \\
\hline 40778 & & -1.60 & 0.100 & 4.50 & 31.0 & 3.5 & 2.118 & 2.118 & 0.055 & 0.061 & 2.297 & 2.201 \\
\hline 42592 & & -1.96 & 0.100 & 4.00 & 23.6 & 1.5 & 2.142 & 2.124 & 0.030 & 0.032 & 2.372 & 2.249 \\
\hline 44605 & & -2.09 & 0.160 & 5.00 & 38.0 & 3.4 & 2.091 & 2.106 & 0.086 & 0.075 & 2.177 & 2.149 \\
\hline 44716 & & -1.02 & 0.100 & 2.50 & $<3.5$ & $\ldots$ & $<0.314$ & $\ldots$ & & $\ldots$ & $<0.353$ & $<0.310$ \\
\hline 46516 & & -2.88 & 0.040 & 3.85 & 19.3 & 1.3 & 2.181 & 2.155 & 0.031 & 0.033 & 2.287 & 2.096 \\
\hline 47480 & $\mathrm{~s}$ & -2.79 & 0.290 & 3.85 & 19.6 & 2.6 & 2.147 & 2.172 & 0.061 & 0.061 & 2.598 & 2.193 \\
\hline 48152 & $?$ & -2.28 & 0.170 & 3.85 & 24.6 & 1.2 & 2.281 & 2.249 & 0.027 & 0.029 & 2.836 & 2.654 \\
\hline 52771 & $\mathrm{x}$ & -1.82 & 0.030 & 5.00 & 45.0 & 6.9 & 2.145 & 2.163 & 0.084 & 0.095 & 2.255 & 2.181 \\
\hline 53070 & $?$ & -1.55 & 0.080 & 4.20 & 35.8 & 1.6 & 2.209 & 2.211 & 0.026 & 0.050 & 2.319 & 2.222 \\
\hline 55022 & $*$ & -1.29 & 0.130 & 3.85 & $<3.0$ & $\ldots$ & $<1.332$ & $<1.313$ & $\ldots$ & $\ldots$ & $<1.544$ & $<2.391$ \\
\hline 59109 & & -2.23 & 0.280 & 4.00 & 25.3 & 1.0 & 2.304 & 2.268 & 0.059 & 0.057 & 2.410 & 2.292 \\
\hline 59376 & & -2.20 & 0.120 & 4.20 & 29.0 & 3.1 & 2.117 & 2.114 & 0.020 & 0.019 & 2.226 & 2.174 \\
\hline 60632 & $\mathrm{x}$ & -1.82 & 0.210 & 4.00 & 31.7 & 1.3 & 2.204 & 2.212 & 0.020 & 0.021 & 2.294 & 2.280 \\
\hline 61361 & $?$ & -2.63 & 0.040 & 4.20 & 36.0 & 2.1 & 2.253 & 2.249 & 0.027 & 0.063 & 2.411 & 2.308 \\
\hline 61545 & $*$ & -2.23 & 0.070 & 4.00 & 27.0 & 3.5 & 2.159 & 2.147 & 0.062 & 0.075 & 2.235 & 2.182 \\
\hline 61802 & & -1.22 & 0.150 & 3.50 & 13.6 & 3.5 & 1.310 & 1.393 & 0.108 & 0.140 & 1.366 & 1.309 \\
\hline 62747 & & -1.34 & 0.150 & 3.00 & 17.2 & 3.5 & 1.268 & 1.381 & 0.091 & 0.109 & 1.166 & 1.040 \\
\hline 63559 & & -0.93 & 0.100 & 4.20 & 37.0 & 2.0 & 2.119 & 2.142 & 0.031 & 0.033 & 2.375 & 2.005 \\
\hline 66354 & $*$ & -1.43 & 0.100 & 4.50 & 8.0 & 2.0 & 1.008 & 1.088 & 0.101 & 0.129 & 1.082 & 1.022 \\
\hline 66673 & $?$ & -3.38 & 0.130 & 3.85 & 25.8 & 4.0 & 2.347 & 2.316 & 0.074 & 0.089 & 2.292 & 2.219 \\
\hline 67655 & & -1.03 & 0.100 & 4.50 & $<6.0$ & $\ldots$ & $<1.167$ & $<1.245$ & $\ldots$ & $\ldots$ & $<1.167$ & $<1.193$ \\
\hline 67863 & & -0.88 & 0.100 & 3.50 & $<5.0$ & $\ldots$ & $<1.283$ & $<1.338$ & $\ldots$ & $\ldots$ & $<1.657$ & $<1.368$ \\
\hline 68321 & $\mathrm{x}$ & -2.34 & 0.050 & 3.85 & 23.0 & 1.5 & 2.172 & 2.151 & 0.031 & 0.033 & 2.221 & 2.218 \\
\hline 68464 & & -1.93 & 0.100 & 3.85 & 40.0 & 2.0 & 2.414 & 2.390 & 0.029 & 0.031 & 2.677 & 2.945 \\
\hline 68592 & & -2.96 & 0.360 & 3.85 & 16.2 & 4.0 & 2.077 & 2.057 & 0.105 & 0.133 & 2.146 & 2.029 \\
\hline
\end{tabular}


Table 6. continued.

\begin{tabular}{|c|c|c|c|c|c|c|c|c|c|c|c|c|}
\hline$\overline{\overline{\text { HIP }}}$ & bin & $\begin{array}{c}{[\mathrm{Fe} / \mathrm{H}]} \\
\operatorname{dex}\end{array}$ & $\begin{array}{c}\sigma(\mathrm{Fe}) \\
\operatorname{dex}\end{array}$ & $\begin{array}{c}\log g \\
\mathrm{~cm} \mathrm{~s}^{-1} \\
\end{array}$ & $\begin{array}{l}E W \\
\mathrm{~mA}\end{array}$ & $\begin{array}{l} \pm 1 \sigma \\
\mathrm{m \AA}\end{array}$ & $\begin{array}{c}A(\mathrm{Li})_{\text {LTE }} \\
\text { dex }\end{array}$ & $\begin{array}{c}A(\mathrm{Li})_{\mathrm{NLTE}} \\
\operatorname{dex}\end{array}$ & $\begin{array}{l}+1 \sigma \\
\operatorname{dex}\end{array}$ & $\begin{array}{l}-1 \sigma \\
\operatorname{dex}\end{array}$ & $\begin{array}{c}A(\mathrm{Li})_{\mathrm{S} 98} \\
\operatorname{dex}\end{array}$ & $\begin{array}{c}A(\mathrm{Li})_{\mathrm{H} 97} \\
\operatorname{dex}\end{array}$ \\
\hline 71458 & & -2.32 & 0.150 & 3.50 & 11.4 & 3.5 & 1.206 & 1.289 & 0.130 & 0.159 & 1.277 & \\
\hline 72461 & $*$ & -2.52 & 0.230 & 4.50 & 30.0 & 3.6 & 2.240 & 2.220 & 0.059 & 0.066 & 2.258 & 2.158 \\
\hline 72561 & $*$ & -1.66 & 0.150 & 4.50 & $<6.3$ & $\ldots$ & $<1.672$ & $<1.639$ & $\ldots$ & $\ldots$ & $<1.758$ & $<1.590$ \\
\hline 72920 & & -2.84 & 0.040 & 4.00 & 21.3 & 2.9 & 2.209 & 2.183 & 0.068 & 0.070 & 2.142 & 2.047 \\
\hline 73385 & $\mathrm{v}$ & -1.62 & 0.180 & 3.50 & 53.0 & 3.5 & 2.376 & 2.383 & 0.042 & 0.043 & 2.593 & 2.219 \\
\hline 74079 & & -1.60 & 0.150 & 3.50 & 44.0 & 3.5 & 2.221 & 2.243 & 0.047 & 0.049 & 2.379 & 2.245 \\
\hline 76059 & & -1.70 & 0.070 & 3.50 & 53.7 & 4.5 & 2.212 & 2.248 & 0.051 & 0.054 & 2.212 & 2.074 \\
\hline 76976 & $\mathrm{~V}$ & -2.48 & 0.080 & 3.50 & 47.2 & 1.8 & 2.260 & 2.278 & 0.024 & 0.024 & 2.260 & 2.228 \\
\hline 78640 & & -1.56 & 0.080 & 4.00 & 28.0 & 4.8 & 2.081 & 2.089 & 0.081 & 0.094 & 2.131 & 2.177 \\
\hline 81276 & $*$ & -1.50 & 0.150 & 3.85 & $<6.0$ & $\ldots$ & $<1.740$ & $<1.705$ & & $\ldots$ & $<1.808$ & $<1.876$ \\
\hline 86443 & $\mathrm{x}$ & -2.32 & 0.210 & 4.50 & 32.7 & 1.3 & 2.065 & 2.070 & 0.021 & 0.021 & 2.065 & 2.324 \\
\hline 86694 & & -1.76 & 0.160 & 3.85 & 41.0 & 2.1 & 2.565 & 2.522 & 0.031 & 0.032 & 2.565 & 2.465 \\
\hline 87101 & & -1.38 & 0.150 & 4.00 & 43.5 & 1.5 & 2.121 & 2.156 & 0.020 & 0.021 & 2.121 & 1.783 \\
\hline 87467 & & -2.57 & 0.040 & 4.00 & 25.5 & 3.0 & 2.274 & 2.246 & 0.057 & 0.067 & 2.348 & 2.268 \\
\hline 87693 & $\mathrm{x}$ & -2.09 & 0.120 & 4.00 & 27.5 & 2.2 & 2.221 & 2.201 & 0.045 & 0.041 & 2.602 & 2.424 \\
\hline 88010 & & -1.36 & 0.120 & 4.00 & 22.1 & 2.1 & 1.599 & 1.668 & 0.041 & 0.046 & 1.599 & 1.702 \\
\hline 88827 & & -2.40 & 0.100 & 4.00 & 21.5 & 2.2 & 2.350 & 2.307 & 0.048 & 0.052 & 2.350 & 2.420 \\
\hline 89554 & & -1.60 & 0.150 & 4.20 & 40.0 & 3.8 & 2.442 & 2.419 & 0.054 & 0.060 & 2.890 & 2.516 \\
\hline 95333 & & -1.70 & 0.300 & 4.50 & 40.6 & 4.5 & 2.242 & 2.241 & 0.076 & 0.085 & 2.620 & 2.285 \\
\hline 96115 & $\mathrm{x}$ & -2.42 & 0.180 & 3.85 & 25.3 & 1.1 & 2.248 & 2.223 & 0.021 & 0.028 & 2.248 & 2.473 \\
\hline 98020 & $\mathrm{x}$ & -1.73 & 0.090 & 5.00 & 18.8 & 3.4 & 1.576 & 1.609 & 0.082 & 0.100 & 1.576 & 1.607 \\
\hline 98532 & & -1.30 & 0.150 & 3.50 & 42.0 & 1.6 & 2.113 & 2.152 & 0.022 & 0.023 & 2.113 & 2.243 \\
\hline 98989 & & -1.71 & 0.080 & 4.00 & 29.8 & 2.6 & 2.092 & 2.102 & 0.043 & 0.046 & 2.088 & 2.357 \\
\hline 99423 & & -1.54 & 0.070 & 3.50 & 79.2 & 2.0 & 2.643 & 2.620 & 0.020 & 0.021 & 2.643 & 2.751 \\
\hline 100568 & & -1.15 & 0.100 & 4.20 & 28.8 & 3.4 & 1.872 & 1.915 & 0.055 & 0.061 & 2.098 & 2.085 \\
\hline 100682 & $?$ & -2.83 & 0.030 & 4.50 & $<2.0$ & $\ldots$ & $<1.108$ & $<1.088$ & & $\ldots$ & $<1.108$ & $<1.249$ \\
\hline 100792 & & -1.45 & 0.110 & 4.00 & 28.8 & 0.9 & 2.001 & 2.021 & 0.015 & 0.015 & 2.487 & 1.685 \\
\hline 102337 & & -2.06 & 0.030 & 4.00 & 29.3 & 3.1 & 2.326 & 2.295 & 0.053 & 0.062 & 2.524 & 2.294 \\
\hline 102718 & s & -1.80 & 0.100 & 3.85 & 29.0 & 7.9 & 1.972 & 1.995 & 0.154 & 0.158 & 2.350 & 2.237 \\
\hline 104660 & $\mathrm{x}$ & -0.80 & 0.150 & 3.50 & 5.0 & 3.3 & 1.038 & 1.098 & 0.227 & 0.477 & 2.364 & 1.946 \\
\hline 104658 & & -1.28 & 0.100 & 4.20 & 25.2 & 0.6 & 1.886 & 1.912 & 0.013 & 0.012 & 1.038 & 1.211 \\
\hline 106468 & & -2.63 & 0.150 & 4.00 & 29.8 & 1.4 & 2.259 & 2.243 & 0.024 & 0.025 & 2.480 & 2.294 \\
\hline 109558 & $*$ & -1.73 & 0.200 & 4.00 & 28.1 & 2.2 & 2.103 & 2.101 & 0.038 & 0.041 & 2.350 & 2.260 \\
\hline 110140 & & -1.25 & 0.070 & 3.85 & 37.0 & 7.4 & 2.219 & 2.229 & 0.115 & 0.138 & 2.509 & 2.315 \\
\hline 111195 & $*$ & -1.39 & 0.090 & 4.50 & 35.0 & 2.6 & 2.158 & 2.163 & 0.043 & 0.064 & 2.389 & 2.160 \\
\hline 111372 & & -2.08 & 0.300 & 3.50 & 31.0 & 4.5 & 2.297 & 2.279 & 0.096 & 0.086 & 2.314 & 2.331 \\
\hline 114271 & & -1.83 & 0.130 & 4.00 & 29.0 & 1.4 & 2.351 & 2.316 & 0.024 & 0.026 & 2.393 & 2.306 \\
\hline 114962 & $?$ & -1.52 & 0.100 & 3.85 & 39.1 & 1.3 & 2.231 & 2.245 & 0.019 & 0.020 & 2.364 & 2.277 \\
\hline 115167 & & -1.81 & 0.310 & 3.85 & 30.5 & 3.5 & 2.178 & 2.173 & 0.058 & 0.068 & 2.392 & 2.231 \\
\hline 115704 & * & -2.00 & 0.060 & 4.50 & 29.0 & 2.1 & 2.163 & 2.146 & 0.041 & 0.038 & 2.163 & 2.399 \\
\hline \multicolumn{13}{|c|}{ Sample \#2: the $\beta$ sample } \\
\hline 484 & & -1.23 & 0.150 & 3.00 & 12.1 & 3.5 & 0.953 & 1.101 & & & 0.949 & \\
\hline 3554 & & -2.87 & 0.150 & 3.00 & 17.7 & 3.5 & 1.018 & 1.153 & 0.087 & 0.104 & 1.044 & 1.238 \\
\hline 4343 & & -2.08 & 0.150 & 3.00 & 9.1 & 3.5 & 0.766 & 0.912 & 0.150 & 0.219 & 0.878 & 0.680 \\
\hline 8314 & $?$ & -1.68 & 0.090 & 4.00 & 27.0 & 3.0 & 2.378 & 2.337 & 0.053 & 0.058 & 2.262 & 2.523 \\
\hline 13749 & & -1.62 & 0.150 & 3.00 & 14.6 & 3.5 & 0.876 & 1.039 & 0.102 & 0.128 & 0.901 & 0.870 \\
\hline 17001 & & -2.70 & 0.150 & 3.00 & 12.6 & 3.5 & 1.054 & 1.171 & 0.115 & 0.150 & 1.058 & 0.926 \\
\hline 21609 & $\mathrm{~s}$ & -1.60 & 0.005 & 4.50 & 21.0: & 3.0 & 1.348: & 1.434: & 0.065 & 0.074 & 1.465: & 1.357: \\
\hline
\end{tabular}


Table 6. continued.

\begin{tabular}{|c|c|c|c|c|c|c|c|c|c|c|c|c|}
\hline HIP & bin & $\begin{array}{c}\mathrm{Fe} / \mathrm{H}] \\
\operatorname{dex}\end{array}$ & $\begin{array}{c}\sigma(\mathrm{Fe}) \\
\operatorname{dex}\end{array}$ & $\begin{array}{c}\log g \\
\mathrm{~cm} \mathrm{~s}^{-1}\end{array}$ & $\begin{array}{l}E W \\
\mathrm{~m} \AA\end{array}$ & $\begin{array}{l} \pm 1 \sigma \\
\mathrm{mA}\end{array}$ & $\begin{array}{c}A(\mathrm{Li})_{\mathrm{LTE}} \\
\text { dex }\end{array}$ & $\begin{array}{c}A(\mathrm{Li})_{\text {NLTE }} \\
\text { dex }\end{array}$ & $\begin{array}{l}+1 \sigma \\
\operatorname{dex}\end{array}$ & $\begin{array}{l}-1 \sigma \\
\operatorname{dex}\end{array}$ & $\begin{array}{c}A(\mathrm{Li})_{\mathrm{S} 98} \\
\text { dex }\end{array}$ & $\begin{array}{c}A(\mathrm{Li})_{\mathrm{H} 97} \\
\text { dex }\end{array}$ \\
\hline 25659 & $\mathrm{v}$ & -2.00 & 0.100 & 4.50 & 43.9 & 6.4 & 2.249 & 2.245 & 0.082 & 0.092 & 2.248 & 2.243 \\
\hline 30668 & & -1.40 & 0.110 & 3.00 & 9.9 & 3.5 & 1.154 & 1.253 & 0.141 & 0.199 & 1.079 & 1.167 \\
\hline 49371 & & -1.75 & 0.150 & 3.00 & 24.5 & 3.5 & 1.328 & 1.457 & 0.068 & 0.076 & 1.294 & 1.354 \\
\hline 57244 & $*$ & -2.80 & 0.100 & 5.00 & 34.0 & 2.2 & 1.864 & 1.901 & 0.019 & 0.039 & 1.961 & 1.825 \\
\hline 57983 & & -2.29 & 0.150 & 3.00 & 10.2 & 3.5 & 0.994 & 1.121 & 0.138 & 0.186 & 1.104 & 0.835 \\
\hline 58357 & & -1.71 & 0.150 & 3.00 & $<4.0$ & ... & $<0.237$ & ... & $\ldots$ & $\ldots$ & $<0.248$ & $<0.301$ \\
\hline 60719 & & -2.32 & 0.150 & 3.00 & $<4.0$ & $\ldots$ & $<0.687$ & $<0.789$ & .. & $\ldots$ & $<0.791$ & $<0.638$ \\
\hline 63385 & & -1.83 & 0.150 & 3.00 & 21.3 & 3.5 & 1.074 & 1.234 & 0.076 & 0.088 & 1.194 & 1.065 \\
\hline 65206 & $*$ & -2.60 & 0.100 & 3.50 & 30.0 & 2.9 & 2.050 & 2.070 & 0.048 & 0.057 & 2.048 & 2.024 \\
\hline 92775 & & -2.18 & 0.150 & 3.50 & 20.7 & 4.2 & 1.759 & 1.799 & 0.090 & 0.109 & 1.773 & 1.148 \\
\hline 99267 & & -2.12 & 0.020 & 4.50 & 45.0 & 4.5 & 2.269 & 2.265 & 0.058 & 0.062 & 2.226 & 2.261 \\
\hline 114502 & & -1.87 & 0.150 & 3.00 & 12.1 & 3.5 & 0.911 & 1.060 & 0.119 & 0.158 & 1.033 & 0.853 \\
\hline 117522 & $*$ & -2.40 & 0.100 & 5.00 & 28.0 & 2.9 & 1.834 & 1.865 & 0.048 & 0.053 & 2.150 & 1.889 \\
\hline \multicolumn{13}{|c|}{ Sample \#3: the $u b v y$ sample } \\
\hline 12807 & & -2.87 & 0.220 & 4.50 & 22.9 & 3.0 & 1.918 & 1.929 & 0.066 & 0.068 & 2.676 & 1.940 \\
\hline 83320 & & -2.56 & 0.150 & 3.50 & $<5.0$ & $\ldots$ & $<1.265$ & 1.287 & . & $\ldots$ & $<1.603$ & $<1.167$ \\
\hline 87062 & & -1.67 & 0.230 & 4.50 & 31.5 & 4.0 & 2.109 & 2.111 & 0.088 & 0.069 & 2.109 & 2.106 \\
\hline 91129 & $*$ & -2.96 & 0.100 & 4.50 & 27.3 & 2.5 & 2.208 & 2.191 & 0.045 & 0.054 & 2.899 & 2.224 \\
\hline 103337 & & -2.07 & 0.150 & 3.00 & 25.8 & 3.5 & 1.025 & 1.197 & 0.069 & 0.072 & 1.179 & 0.877 \\
\hline 104191 & & -2.99 & 0.120 & 3.00 & 9.0 & 1.7 & 0.959 & 1.070 & 0.084 & 0.100 & 0.840 & 1.435 \\
\hline
\end{tabular}

[?] Identifies a suspected binary (Latham et al. 2002; Carney et al. 1994, 2003). * Identifies a confirmed single- or double-lined binary (from Latham et al. 2002; Carney et al. 1994, 2003). 
Table 8. Quantities extracted from the HIPPARCOS catalogue for our sample stars $((B-V)$ and $E(B-V)$ are given in Table 4$)$.

\begin{tabular}{|c|c|c|c|c|c|}
\hline HIP & $\begin{array}{c}V \\
\operatorname{mag}\end{array}$ & $\begin{array}{l}\text { Plx } \\
\text { (mas) }\end{array}$ & $\begin{array}{l}\text { e_Plx } \\
\text { (mas) }\end{array}$ & $\begin{array}{c}d \\
(\mathrm{pc})\end{array}$ & VI \\
\hline 911 & 11.80 & 6.13 & 5.67 & 163.13 & 0.64 \\
\hline 3026 & 9.25 & 9.57 & 1.38 & 104.49 & 0.54 \\
\hline 3446 & 12.10 & 15.15 & 3.24 & 66.01 & 0.58 \\
\hline 3554 & 9.02 & 4.81 & 1.40 & 207.90 & 0.79 \\
\hline 3564 & 10.60 & 2.07 & 2.16 & 483.09 & 0.67 \\
\hline 4343 & 9.62 & 0.17 & 1.60 & 5882.35 & 0.78 \\
\hline 8314 & 9.94 & 6.46 & 1.27 & 154.80 & 0.48 \\
\hline 8572 & 10.34 & 3.22 & 1.75 & 310.56 & 0.50 \\
\hline 10140 & 8.76 & 17.66 & 1.29 & 56.63 & 0.65 \\
\hline 11952 & 9.77 & 8.67 & 1.81 & 115.34 & 0.51 \\
\hline 12529 & 10.17 & 5.22 & 1.81 & 191.57 & 0.59 \\
\hline 12807 & 11.41 & 8.64 & 3.04 & 115.74 & 0.67 \\
\hline 13749 & 10.31 & 2.07 & 1.74 & 483.09 & 0.81 \\
\hline 14594 & 8.04 & 25.85 & 1.14 & 38.68 & 0.56 \\
\hline 16072 & 10.81 & 6.99 & 2.19 & 143.06 & 0.62 \\
\hline 17001 & 9.92 & 4.42 & 1.75 & 226.24 & 0.71 \\
\hline 18082 & 9.97 & 4.91 & 1.69 & 203.67 & 0.60 \\
\hline 18802 & 8.11 & 19.02 & 0.87 & 52.58 & 0.64 \\
\hline 19797 & 9.23 & 12.84 & 1.33 & 77.88 & 0.42 \\
\hline 21609 & 9.85 & 17.00 & 0.98 & 58.82 & 0.70 \\
\hline 22632 & 9.13 & 15.55 & 1.20 & 64.31 & 0.57 \\
\hline 23344 & 9.79 & 7.80 & 2.09 & 128.21 & 0.48 \\
\hline 24316 & 9.43 & 9.43 & 14.55 & 1.01 & 0.04 \\
\hline 25659 & 11.61 & 7.69 & 2.47 & 130.04 & 0.58 \\
\hline 28887 & 10.31 & 1.80 & 1.95 & 555.56 & 0.74 \\
\hline 29759 & 8.92 & 5.78 & 1.31 & 173.01 & 0.68 \\
\hline 30098 & 10.74 & 0.68 & 2.31 & 1470.59 & 0.79 \\
\hline 30668 & 8.00 & 7.34 & 0.96 & 136.24 & 0.76 \\
\hline 32567 & 10.32 & 3.98 & 1.81 & 251.26 & 0.51 \\
\hline 36174 & 10.73 & 6.19 & 2.17 & 161.55 & 0.51 \\
\hline 36430 & 10.41 & 1.20 & 2.36 & 833.33 & 0.61 \\
\hline 36513 & 10.80 & 3.07 & 2.32 & 325.73 & 0.41 \\
\hline 37671 & 10.39 & 3.08 & 5.42 & 324.68 & 0.61 \\
\hline 38541 & 8.27 & 35.29 & 1.04 & 28.34 & 0.69 \\
\hline 40778 & 9.73 & 10.36 & 1.47 & 96.53 & 0.56 \\
\hline 42592 & 9.67 & 7.26 & 1.32 & 137.74 & 0.50 \\
\hline 44605 & 11.60 & 10.26 & 3.45 & 97.47 & 0.60 \\
\hline 44716 & 7.68 & 2.66 & 1.29 & 375.94 & 0.83 \\
\hline 46516 & 11.15 & 1.04 & 2.79 & 961.54 & 0.46 \\
\hline 47480 & 10.18 & 1.20 & 4.79 & 833.33 & 0.47 \\
\hline 48152 & 8.33 & 12.44 & 1.06 & 80.39 & 0.46 \\
\hline 49371 & 8.97 & 4.38 & 1.30 & 228.31 & 0.77 \\
\hline 52771 & 10.26 & 10.55 & 1.75 & 94.79 & 0.58 \\
\hline 53070 & 8.21 & 19.23 & 1.13 & 52.00 & 0.57 \\
\hline 55022 & 9.21 & 7.69 & 1.23 & 130.04 & 0.49 \\
\hline 57244 & 12.02 & 4.21 & 3.05 & 237.53 & 0.61 \\
\hline 57983 & 9.59 & 2.98 & 1.35 & 335.57 & 0.78 \\
\hline 58357 & 8.35 & 5.55 & 1.20 & 180.18 & 0.87 \\
\hline 59109 & 10.00 & 5.75 & 1.55 & 173.91 & 0.48 \\
\hline 59376 & 11.09 & 4.76 & 2.01 & 210.08 & 0.52 \\
\hline 60632 & 9.66 & 10.95 & 1.29 & 91.32 & 0.51 \\
\hline 60719 & 8.03 & 4.53 & 1.06 & 220.75 & 0.68 \\
\hline 61361 & 12.01 & 4.91 & 3.26 & 203.67 & 0.52 \\
\hline 61545 & 10.89 & 5.35 & 1.94 & 186.92 & 0.52 \\
\hline
\end{tabular}

Table 8. continued.

\begin{tabular}{cccccc}
\hline \hline & & & & & \\
HIP & $V$ & Plx & e_Plx & $d$ & VI \\
& mag & (mas) & (mas) & $(\mathrm{pc})$ & \\
\hline & & & & & \\
61802 & 9.79 & 4.55 & 2.13 & 219.78 & 0.72 \\
62747 & 7.97 & 3.29 & 1.11 & 303.95 & 0.83 \\
63385 & 9.72 & 2.36 & 1.49 & 423.73 & 0.79 \\
63559 & 8.04 & 18.51 & 1.12 & 54.02 & 0.62 \\
65206 & 11.66 & 1.59 & 1.72 & 628.93 & 0.55 \\
66354 & 10.85 & 12.26 & 2.28 & 81.57 & 0.73 \\
66673 & 11.47 & 1.88 & 2.90 & 531.91 & 0.47 \\
67655 & 7.97 & 40.02 & 1.00 & 24.99 & 0.72 \\
67863 & 9.02 & 16.73 & 1.35 & 59.77 & 0.67 \\
68321 & 10.05 & 5.37 & 1.60 & 186.22 & 0.48 \\
68464 & 8.73 & 9.77 & 1.32 & 102.35 & 0.53 \\
68592 & 11.13 & 2.88 & 3.10 & 347.22 & 0.46 \\
71458 & 8.02 & 5.96 & 1.32 & 167.79 & 0.70 \\
72461 & 9.73 & 10.28 & 1.42 & 97.28 & 0.51 \\
72561 & 11.05 & 7.01 & 2.43 & 142.65 & 0.48 \\
72920 & 11.00 & 4.81 & 2.09 & 207.90 & 0.47 \\
73385 & 8.55 & 10.85 & 1.14 & 92.17 & 0.58 \\
74079 & 7.67 & 16.80 & 1.11 & 59.52 & 0.64 \\
76059 & 8.43 & 11.07 & 1.28 & 90.33 & 0.64 \\
76976 & 7.20 & 17.44 & 0.97 & 57.34 & 0.56 \\
78640 & 9.86 & 8.03 & 1.12 & 124.53 & 0.55 \\
81276 & 11.24 & 2.22 & 1.48 & 450.45 & 0.42 \\
83320 & 11.46 & 2.14 & 2.87 & 467.29 & 0.53 \\
86443 & 9.94 & 8.35 & 1.64 & 119.76 & 0.53 \\
86694 & 8.72 & 8.66 & 1.25 & 115.47 & 0.53 \\
87062 & 10.60 & 10.34 & 2.20 & 96.71 & 0.67 \\
87101 & 9.68 & 10.61 & 1.53 & 94.25 & 0.73 \\
87467 & 10.35 & 5.91 & 1.17 & 169.20 & 0.59 \\
87693 & 9.77 & 6.47 & 7.85 & 154.56 & 0.51 \\
88010 & 9.63 & 11.88 & 2.21 & 84.18 & 0.68 \\
88827 & 11.09 & 5.42 & 2.32 & 184.50 & 0.52 \\
89554 & 8.22 & 16.09 & 1.04 & 62.15 & 0.51 \\
91129 & 11.39 & 3.59 & 2.15 & 278.55 & 0.52 \\
92775 & 9.96 & 1.15 & 1.04 & 869.57 & 0.71 \\
95333 & 9.68 & 11.31 & 1.76 & 88.42 & 0.54 \\
96115 & 9.37 & 6.93 & 1.48 & 144.30 & 0.46 \\
98020 & 8.83 & 25.32 & 1.17 & 39.49 & 0.67 \\
98532 & 7.72 & 14.76 & 1.10 & 67.75 & 0.64 \\
98989 & 10.46 & 5.31 & 1.83 & 188.32 & 0.58 \\
99267 & 10.11 & 12.04 & 1.13 & 83.06 & 0.58 \\
99423 & 8.89 & 9.38 & 1.24 & 106.61 & 0.56 \\
100568 & 8.65 & 22.88 & 1.24 & 43.71 & 0.63 \\
100682 & 10.83 & 7.49 & 1.73 & 133.51 & 0.49 \\
100792 & 8.33 & 17.94 & 1.24 & 55.74 & 0.62 \\
102337 & 9.94 & 7.32 & 1.56 & 136.61 & 0.50 \\
\hline & & & & &
\end{tabular}


C. Charbonnel and F. Primas: The lithium content of the Galactic Halo stars, Online Material p 14

Table 8. continued.

\begin{tabular}{cccccc}
\hline \hline HIP & $\begin{array}{c}V \\
\text { mag }\end{array}$ & $\begin{array}{c}\text { Plx } \\
(\text { mas })\end{array}$ & $\begin{array}{c}\text { e_Plx } \\
(\text { mas })\end{array}$ & $\begin{array}{c}d \\
(\mathrm{pc})\end{array}$ & VI \\
\hline & & & & & \\
102718 & 9.59 & 1.64 & 4.29 & 609.76 & 0.61 \\
103337 & 10.21 & 1.48 & 1.25 & 675.68 & 0.90 \\
104191 & 9.09 & 3.20 & 1.25 & 312.50 & 0.65 \\
104658 & 8.29 & 4.38 & 1.18 & 228.31 & 0.15 \\
104660 & 8.06 & 17.95 & 1.44 & 55.71 & 0.67 \\
106468 & 10.56 & 6.45 & 1.98 & 155.04 & 0.47 \\
109558 & 9.46 & 8.43 & 1.42 & 118.62 & 0.53 \\
110140 & 10.38 & 3.97 & 2.01 & 251.89 & 0.56 \\
111195 & 10.71 & 8.99 & 2.13 & 111.23 & 0.59 \\
111372 & 9.65 & 5.68 & 1.54 & 176.06 & 0.50 \\
114271 & 8.25 & 14.33 & 1.20 & 69.78 & 0.48 \\
114502 & 8.94 & 3.51 & 1.42 & 284.90 & 0.77 \\
114962 & 8.16 & 12.04 & 2.41 & 83.06 & 0.62 \\
115167 & 10.21 & 3.26 & 2.20 & 306.75 & 0.53 \\
115704 & 10.49 & 8.85 & 1.43 & 112.99 & 0.54 \\
117522 & 11.34 & 4.48 & 2.41 & 223.21 & 4.52 \\
\hline
\end{tabular}


Table 9. Characteristics and evolutionary status of the sample stars.

\begin{tabular}{|c|c|c|c|c|c|c|c|c|c|}
\hline HIP & $M_{V}$ & $\begin{array}{c}M_{V} \\
\text { dered }\end{array}$ & $\mathrm{BC}$ & $M$ bol & $\log \left(\frac{L}{L_{\odot}}\right)$ & $\begin{array}{c}\log \left(\frac{L}{L_{\odot}}\right) \\
\text { dered }\end{array}$ & e $\_\log \left(\frac{L}{L_{\odot}}\right)$ & $\log g$ & Status $^{s}$ \\
\hline 911 & 5.74 & 5.74 & -0.176 & 5.56 & -0.32 & -0.32 & 0.80 & 4.50 & 5 \\
\hline 3026 & 4.15 & 4.15 & -0.169 & 3.99 & 0.31 & 0.31 & 0.13 & 3.85 & 5 \\
\hline 3446 & 8.00 & 8.00 & -0.085 & 7.92 & -1.27 & -1.27 & 0.19 & 4.50 & 5 \\
\hline 3554 & 2.43 & 2.34 & 0.170 & 2.60 & 0.86 & 0.89 & 0.26 & 3.00 & 3.5 \\
\hline 3564 & 2.18 & 2.06 & -0.221 & 1.96 & 1.12 & 1.16 & 0.91 & 3.50 & 4 \\
\hline 4343 & -4.23 & -4.25 & -0.228 & -4.46 & 3.68 & 3.69 & 8.18 & 3.00 & - \\
\hline 8314 & 3.99 & 3.84 & -0.139 & 3.85 & 0.36 & 0.42 & 0.18 & 4.00 & 5 \\
\hline 8572 & 2.88 & 2.79 & -0.112 & 2.77 & 0.79 & 0.83 & 0.47 & 3.85 & 4 \\
\hline 10140 & 4.99 & 4.99 & -0.218 & 4.78 & -0.01 & -0.01 & 0.07 & 4.20 & 5 \\
\hline 11952 & 4.46 & 4.44 & -0.125 & 4.34 & 0.17 & 0.17 & 0.19 & 4.50 & 5 \\
\hline 12529 & 3.76 & 3.69 & -0.154 & 3.60 & 0.46 & 0.48 & 0.30 & 3.85 & 4 \\
\hline 12807 & 6.09 & 6.05 & -0.164 & 5.93 & -0.47 & -0.45 & 0.31 & 4.50 & 5 \\
\hline 13749 & 1.89 & 1.84 & -0.255 & 1.63 & 1.25 & 1.26 & 0.73 & 3.00 & 3.5 \\
\hline 14594 & 5.10 & 5.10 & -0.142 & 4.96 & -0.08 & -0.08 & 0.06 & 4.50 & 5 \\
\hline 16072 & 5.03 & 4.81 & -0.198 & 4.83 & -0.04 & 0.06 & 0.28 & 4.50 & 5 \\
\hline 17001 & 3.15 & 3.14 & -0.187 & 2.96 & 0.72 & 0.72 & 0.35 & 3.00 & 3.5 \\
\hline 18082 & 3.43 & 3.25 & -0.158 & 3.27 & 0.59 & 0.66 & 0.30 & 3.50 & 4 \\
\hline 18802 & 4.51 & 4.51 & -0.214 & 4.29 & 0.18 & 0.18 & 0.06 & 3.50 & 5 \\
\hline 19797 & 4.77 & 4.77 & -0.115 & 4.66 & 0.04 & 0.04 & 0.10 & 4.00 & 5 \\
\hline 21609 & 6.00 & 5.94 & -0.234 & 5.77 & -0.411 & -0.38 & 0.06 & 4.50 & 5 \\
\hline 22632 & 5.09 & 5.07 & -0.177 & 4.91 & -0.06 & -0.06 & 0.08 & 4.50 & 5 \\
\hline 23344 & 4.25 & 4.19 & -0.095 & 4.16 & 0.24 & 0.26 & 0.24 & 4.00 & 5 \\
\hline 24316 & 5.24 & 5.24 & -0.181 & 5.06 & -0.13 & -0.13 & 0.07 & 4.50 & 5 \\
\hline 25659 & 6.04 & 6.04 & -0.150 & 5.89 & -0.46 & -0.46 & 0.28 & 4.50 & 5 \\
\hline 28887 & 1.59 & 1.52 & -0.207 & 1.38 & 1.35 & 1.37 & 0.94 & 3.00 & 3.5 \\
\hline 29759 & 2.73 & 2.57 & -0.196 & 2.53 & 0.89 & 0.95 & 0.20 & 3.50 & 3.5 \\
\hline 30098 & -0.10 & -0.13 & -0.237 & -0.33 & 2.04 & 2.05 & 2.95 & 3.20 & 3.5 \\
\hline 30668 & 2.33 & 2.20 & -0.221 & 2.11 & 1.06 & 1.11 & 0.12 & 3.00 & 3.5 \\
\hline 32567 & 3.32 & 3.31 & -0.152 & 3.17 & 0.63 & 0.64 & 0.40 & 3.85 & 4 \\
\hline 36174 & 4.69 & 4.62 & -0.152 & 4.54 & 0.08 & 0.11 & 0.31 & 4.20 & 5 \\
\hline 36430 & 0.81 & 0.69 & -0.162 & 0.64 & 1.64 & 1.69 & 1.71 & 3.50 & 4 \\
\hline 36513 & 3.24 & 3.21 & -0.093 & 3.14 & 0.64 & 0.65 & 0.66 & 3.85 & 4 \\
\hline 37671 & 2.83 & 2.76 & -0.194 & 2.64 & 0.84 & 0.88 & 1.53 & 3.85 & 4 \\
\hline 38541 & 6.01 & 6.01 & -0.230 & 5.78 & -0.41 & -0.41 & 0.05 & 4.50 & 5 \\
\hline 40778 & 4.81 & 4.81 & -0.172 & 4.63 & 0.05 & 0.05 & 0.13 & 4.50 & 5 \\
\hline 42592 & 3.97 & 3.97 & -0.122 & 3.85 & 0.36 & 0.36 & 0.16 & 4.00 & 5 \\
\hline 44605 & 6.66 & 6.66 & -0.158 & 6.50 & -0.70 & -0.70 & 0.29 & 5.00 & 5 \\
\hline 44716 & -0.20 & -0.26 & -0.269 & -0.46 & 2.09 & 2.11 & 0.42 & 2.50 & 3 \\
\hline 46516 & 1.24 & 1.16 & -0.091 & 1.14 & 1.44 & 1.47 & 2.33 & 3.85 & 4 \\
\hline 47480 & 0.58 & 0.56 & -0.104 & 0.47 & 1.71 & 1.72 & 3.47 & 3.85 & 4 \\
\hline 48152 & 3.80 & 3.77 & -0.111 & 3.69 & 0.42 & 0.44 & 0.08 & 3.85 & 4.5 \\
\hline 49371 & 2.18 & 2.18 & -0.227 & 1.95 & 1.12 & 1.12 & 0.26 & 3.00 & 3.5 \\
\hline 52771 & 5.38 & 5.38 & -0.150 & 5.23 & -0.19 & -0.19 & 0.15 & 5.00 & 5 \\
\hline 53070 & 4.63 & 4.63 & -0.177 & 4.45 & 0.12 & 0.12 & 0.06 & 4.20 & 5 \\
\hline 55022 & 3.64 & 3.63 & -0.143 & 3.50 & 0.50 & 0.50 & 0.14 & 3.85 & 4.5 \\
\hline 57244 & 5.14 & 5.14 & -0.137 & 5.00 & -0.10 & -0.10 & 0.63 & 5.00 & 5 \\
\hline 57983 & 1.96 & 1.94 & -0.241 & 1.72 & 1.21 & 1.22 & 0.40 & 3.00 & 3.5 \\
\hline
\end{tabular}


C. Charbonnel and F. Primas: The lithium content of the Galactic Halo stars, Online Material p 16

Table 9. continued.

\begin{tabular}{|c|c|c|c|c|c|c|c|c|c|}
\hline HIP & $M_{V}$ & $\begin{array}{c}M_{V} \\
\text { dered }\end{array}$ & $\mathrm{BC}$ & $M$ bol & $\log \left(\frac{L}{L_{\odot}}\right)$ & $\begin{array}{c}\log \left(\frac{L}{L_{\odot}}\right) \\
\text { dered }\end{array}$ & e_Log $\left(\frac{L}{L_{\odot}}\right)$ & $\log g$ & Status $^{s}$ \\
\hline 58357 & 2.07 & 2.07 & -0.298 & 1.77 & 1.19 & 1.19 & 0.19 & 3.00 & 3.5 \\
\hline 59109 & 3.80 & 3.72 & -0.116 & 3.68 & 0.43 & 0.46 & 0.24 & 4.20 & 4.5 \\
\hline 59376 & 4.48 & 4.48 & -0.128 & 4.35 & 0.16 & 0.16 & 0.37 & 4.00 & 5 \\
\hline 60632 & 4.86 & 4.86 & -0.152 & 4.71 & 0.02 & 0.02 & 0.11 & 4.00 & 5 \\
\hline 60719 & 1.31 & 1.31 & -0.165 & 1.15 & 1.44 & 1.44 & 0.21 & 3.00 & 3.5 \\
\hline 61361 & 5.47 & 5.47 & -0.118 & 5.35 & -0.24 & -0.24 & 0.58 & 4.20 & 5 \\
\hline 61545 & 4.53 & 4.53 & -0.128 & 4.40 & 0.14 & 0.14 & 0.32 & 4.00 & 5 \\
\hline 61802 & 3.08 & 3.01 & -0.254 & 2.83 & 0.77 & 0.80 & 0.41 & 3.50 & 3.5 \\
\hline 62747 & 0.56 & 0.28 & -0.269 & 0.29 & 1.79 & 1.90 & 0.30 & 3.00 & 3.5 \\
\hline 63385 & 1.58 & 1.58 & -0.237 & 1.35 & 1.36 & 1.36 & 0.55 & 3.00 & 3.5 \\
\hline 63559 & 4.38 & 4.33 & -0.204 & 4.17 & 0.23 & 0.25 & 0.07 & 4.20 & 5 \\
\hline 65206 & 2.67 & 2.67 & -0.128 & 2.54 & 0.88 & 0.88 & 0.94 & 3.50 & 4 \\
\hline 66354 & 6.29 & 6.28 & -0.249 & 6.04 & -0.52 & -0.51 & 0.17 & 4.50 & 5 \\
\hline 66673 & 2.84 & 2.72 & -0.035 & 2.81 & 0.78 & 0.83 & 1.34 & 3.85 & 4 \\
\hline 67655 & 5.98 & 5.96 & -0.254 & 5.73 & -0.39 & -0.38 & 0.05 & 4.50 & 5 \\
\hline 67863 & 5.14 & 5.09 & -0.228 & 4.91 & -0.06 & -0.04 & 0.08 & 3.50 & 5 \\
\hline 68321 & 3.70 & 3.69 & -0.107 & 3.59 & 0.46 & 0.47 & 0.26 & 3.85 & 4.5 \\
\hline 68464 & 3.68 & 3.58 & -0.131 & 3.55 & 0.48 & 0.52 & 0.12 & 3.85 & 4 \\
\hline 68592 & 3.43 & 3.39 & -0.091 & 3.34 & 0.57 & 0.58 & 0.94 & 3.85 & 4 \\
\hline 71458 & 1.90 & 1.73 & -0.195 & 1.70 & 1.22 & 1.29 & 0.19 & 3.50 & 3.5 \\
\hline 72461 & 4.79 & 4.70 & -0.115 & 4.67 & 0.03 & 0.06 & 0.13 & 4.50 & 5 \\
\hline 72561 & 5.28 & 5.20 & -0.139 & 5.14 & -0.16 & -0.12 & 0.31 & 4.50 & 5 \\
\hline 72920 & 4.41 & 4.26 & -0.093 & 4.32 & 0.17 & 0.23 & 0.38 & 4.00 & 5 \\
\hline 73385 & 3.73 & 3.55 & -0.181 & 3.55 & 0.48 & 0.55 & 0.10 & 3.50 & 4 \\
\hline 74079 & 3.80 & 3.80 & -0.207 & 3.59 & 0.46 & 0.46 & 0.07 & 3.50 & 4 \\
\hline 76059 & 3.65 & 3.49 & -0.207 & 3.44 & 0.52 & 0.59 & 0.11 & 3.50 & 4 \\
\hline 76976 & 3.41 & 3.34 & -0.132 & 3.28 & 0.59 & 0.62 & 0.06 & 3.50 & 4 \\
\hline 78640 & 4.38 & 4.38 & -0.168 & 4.22 & 0.21 & 0.21 & 0.13 & 4.00 & 5 \\
\hline 81276 & 2.97 & 2.97 & -0.115 & 2.86 & 0.76 & 0.76 & 0.58 & 3.85 & 4 \\
\hline 83320 & 3.11 & 2.97 & -0.131 & 2.98 & 0.71 & 0.77 & 1.17 & 3.50 & 4 \\
\hline 86443 & 4.55 & 4.54 & -0.121 & 4.43 & 0.13 & 0.13 & 0.18 & 4.50 & 5 \\
\hline 86694 & 3.41 & 3.17 & -0.131 & 3.28 & 0.59 & 0.68 & 0.13 & 3.85 & 4 \\
\hline 87062 & 5.67 & 5.22 & -0.221 & 5.45 & -0.28 & -0.10 & 0.19 & 4.50 & 5 \\
\hline 87101 & 4.81 & 4.31 & -0.249 & 4.56 & 0.08 & 0.28 & 0.13 & 4.00 & 5 \\
\hline 87467 & 4.21 & 4.09 & -0.143 & 4.06 & 0.27 & 0.323 & 0.18 & 4.00 & 5 \\
\hline 87693 & 3.82 & 3.80 & -0.125 & 3.70 & 0.42 & 0.43 & 1.05 & 4.00 & 4 \\
\hline 88010 & 5.00 & 4.93 & -0.225 & 4.78 & -0.01 & 0.02 & 0.17 & 4.00 & 5 \\
\hline 88827 & 4.76 & 4.40 & -0.160 & 4.60 & 0.06 & 0.20 & 0.37 & 4.00 & 5 \\
\hline 89554 & 4.25 & 4.22 & -0.152 & 4.10 & 0.26 & 0.27 & 0.07 & 4.20 & 5 \\
\hline 91129 & 4.17 & 4.03 & -0.106 & 4.06 & 0.28 & 0.33 & 0.52 & 4.50 & 5 \\
\hline 92775 & 0.26 & 0.26 & -0.212 & 0.05 & 1.88 & 1.8 & 0.79 & 3.50 & 3.5 \\
\hline 95333 & 4.95 & 4.95 & -0.164 & 4.78 & -0.01 & -0.01 & 0.14 & 4.50 & 5 \\
\hline 96115 & 3.57 & 3.55 & -0.102 & 3.47 & 0.51 & 0.52 & 0.19 & 3.85 & 4.5 \\
\hline 98020 & 5.85 & 5.81 & -0.221 & 5.63 & -0.35 & -0.34 & 0.06 & 5.00 & 5 \\
\hline 98532 & 3.57 & 3.55 & -0.207 & 3.36 & 0.56 & 0.56 & 0.08 & 3.50 & 4 \\
\hline 98989 & 4.09 & 4.01 & -0.181 & 3.90 & 0.34 & 0.37 & 0.30 & 4.00 & 5 \\
\hline 99267 & 5.51 & 5.41 & -0.150 & 5.36 & -0.25 & -0.20 & 0.09 & 4.50 & 5 \\
\hline 99423 & 3.75 & 3.72 & -0.172 & 3.58 & 0.47 & 0.48 & 0.12 & 3.50 & 4 \\
\hline 100568 & 5.45 & 5.45 & -0.209 & 5.24 & -0.19 & -0.19 & 0.06 & 4.20 & 5 \\
\hline 100682 & 5.20 & 5.16 & -0.098 & 5.10 & -0.14 & -0.12 & 0.20 & 4.50 & 5 \\
\hline 100792 & 4.60 & 4.60 & -0.198 & 4.40 & 0.14 & 0.14 & 0.07 & 4.00 & 5 \\
\hline 102337 & 4.26 & 4.17 & -0.122 & 4.14 & 0.24 & 0.28 & 0.19 & 4.00 & 5 \\
\hline 102718 & 0.66 & 0.63 & -0.162 & 0.50 & 1.70 & 1.71 & 2.27 & 3.85 & 4 \\
\hline 103337 & 1.06 & 0.96 & -0.328 & 0.73 & 1.61 & 1.65 & 1.09 & 3.00 & 3.5 \\
\hline
\end{tabular}


Table 9. continued.

\begin{tabular}{cccccccccc}
\hline \hline HIP & $M_{V}$ & $\begin{array}{c}M_{V} \\
\text { dered }\end{array}$ & BC & $M$ bol & $\log \left(\frac{L}{L_{\odot}}\right)$ & $\begin{array}{c}\log \left(\frac{L}{L_{\odot}}\right) \\
\text { dered }\end{array}$ & e_Log$\left(\frac{L}{L_{\odot}}\right)$ & $\log g$ & Status $^{s}$ \\
\hline 104191 & 1.62 & 1.62 & 0.465 & 2.08 & 1.07 & 1.07 & 0.34 & 3.00 & 3.5 \\
104658 & 1.50 & 1.50 & -0.115 & 1.38 & 1.35 & 1.35 & 0.24 & 4.20 & 4 \\
104660 & 4.33 & 4.33 & -0.228 & 4.10 & 0.26 & 0.26 & 0.08 & 3.50 & 4 \\
106468 & 4.61 & 4.56 & -0.104 & 4.50 & 0.10 & 0.12 & 0.27 & 4.00 & 5 \\
109558 & 4.09 & 4.06 & -0.160 & 3.93 & 0.33 & 0.34 & 0.15 & 4.00 & 5 \\
110140 & 3.37 & 3.31 & -0.177 & 3.20 & 0.62 & 0.65 & 0.44 & 3.85 & 4 \\
111195 & 5.48 & 5.42 & -0.185 & 5.29 & -0.22 & -0.19 & 0.21 & 4.50 & 5 \\
111372 & 3.42 & 3.39 & -0.122 & 3.30 & 0.58 & 0.59 & 0.24 & 3.50 & 4 \\
114271 & 4.03 & 3.95 & -0.116 & 3.92 & 0.33 & 0.37 & 0.08 & 4.00 & 5 \\
114502 & 1.67 & 1.58 & -0.220 & 1.45 & 1.32 & 1.36 & 0.35 & 3.00 & 3.5 \\
114962 & 3.56 & 3.56 & -0.198 & 3.36 & 0.55 & 0.55 & 0.18 & 3.85 & 4 \\
115167 & 2.78 & 2.74 & -0.131 & 2.64 & 0.84 & 0.86 & 0.59 & 3.85 & 4 \\
115704 & 5.22 & 5.20 & -0.135 & 5.09 & -0.14 & -0.13 & 0.15 & 4.50 & 5 \\
117522 & 4.60 & 4.52 & -0.147 & 4.45 & 0.12 & 0.15 & 0.47 & 5.00 & 5 \\
\hline
\end{tabular}

(s) Status: 5: dwarf - 4.5: turnoff - 4: subgiant - 3.5: base RGB - 3:RGB. (see the text for more details on the adopted definitions of these statuses). 